\title{
Phase-Shifting Point Diffraction Interferometer Having Two Point Light Sources of Single-Mode Optical Fibers
}

\author{
Oshikane Yasushi, Nakano Motohiro and Inoue Haruyuki \\ Graduate School of Engineering, Osaka University
}

Japan

\section{Introduction}

State-of-the-art research fields such as projection optics for Extreme Ultraviolet Lithography (EUVL) (Otaki et al., 2002a; Stulen \& Sweeney, 1999; Goldberg et al., 2004), focusing optics for X-ray microscope (Yamamura \& Takai, 2008; Handa et al., 2008; Yumoto et al., 2008; Matsuyama et al., 2008), and Michelson interferometers for detection of gravitational waves (Ando et al., 2001; Sato et al., 1999) require ultra precise mirrors with large surface areas and extremely low surface figure error (in the sub-nanometer scale). In EUVL, a light wave of $13.5 \mathrm{~nm}$ is used for photoresist exposure, and such an optical system accepts reflection optics instead of refractive optics owing to the short wavelength. In addition, EUV exposure of a dynamic random access memory (DRAM) pattern with a half pitch (hp) of $45 \mathrm{~nm}$ or less requires a numerical aperture (NA) of 0.2 or more. An EUV optical system is designed using $\mathrm{Mo} / \mathrm{Si}$ multilayer mirrors. The surface figure accuracy of the mirror substrate determines the reflectance of the mirror and the long-range error must be less than $0.2 \mathrm{~nm} \mathrm{rms}$. Production of high-precision mirrors with large surface areas is usually supported by highly accurate surface figure measurement methods. For example, the slope integration method (Higashi et al., 2007), three-flat method (Schulz \& Schwider, 1967), and point diffraction interferometry (PDI) (Smartt \& Steel, 1975; Sommargren, 1996; Medecki et al., 1996; Millerd et al., 2004; Otaki et al., 2002b) have been studied and applied to the measurement of mirrors. The slope integration method determines the surface normal vector at each point on the test surface and the resultant angle of inclination is integrated to obtain the absolute surface figure. However, this method requires highly accurate locations of each measurement point. The three-flat method uses three mirrors including the test mirror and measures the relative surface figure between two of the mirrors at a time using conventional optical interferometry. The relative figure data are solved as a set of simultaneous equations to obtain each surface figure. However, this method returns a line profile of the mirrors rather than a whole surface figure. PDI is a novel absolute surface figure measurement system using an optical diffraction wave from a tiny aperture as the reference surface. A conventional Fizeau interferometer uses a substantial reference surface for the measurement and the machining accuracy of the reference results in measurement accuracy of approximately $\lambda / 50$. In contrast, the wavefront accuracy of the optical diffraction wave used in the PDI system has been estimated to be $10^{-5} \lambda$ or less at an NA of 0.2 (Sommargren, 1996). 
Therefore, PDI has a much better reference surface and will enable the realization of ultrahigh precision measurement of the absolute surface figure. Thus, PDI has been adopted as the phase-shifting (PS) method for the analysis of interference patterns (de Groot, 1995a; de Groot, 1995b). In the PDI system, generation of a diffraction wave is dependent on a tiny pinhole or single-mode optical fiber. Nikon has measured spherical or aspherical concave mirrors using a diffraction wave in the visible region emitted from a tiny pinhole (Otaki et al., 2002a; Otaki et al., 2002b; Ota et al., 2001). On the other hand, EUVL researchers at the Lawrence Livermore National Laboratory (LLNL) have reported surface figure measurement data using the PDI system with a single-mode fiber (Sommargren, 1996). In the two types of PS/PDI systems, a diffraction wave radiating from a single tiny hole serves as both a diagnostic wave and a reference wave, the diffraction wave could not be used entirely as the diagnostic wave. This means that the maximum measurable mirror diameter is approximately half the diameter of the diffraction wave. Therefore, we have developed a PS/PDI system with two optical fibers as point sources for large-aperture optics (Oshikane et al., 2003). One fiber acts as a point source for a diagnostic wave, and the other fiber generates a reference wave. The entire diffraction wave will be applied to the measurement of large optics. PDI is suitable for spherical concave mirrors due to its spherical wavefront as the reference surface. When an aspherical mirror is tested, the diagnostic wavefront gets highly distorted and the resultant PDI interferograms do not reflect the surface figure in PS analysis. Therefore, aspherical surface figure measurement by PS/PDI usually requires (1) the stitching method, which connects all valid PS/PDI data in every part of the surface (Ota et al., 2001; Chen et al., 2006), or (2) numerical restoration of the actual wavefront on the mirror surface from the highly distorted PS/PDI data (Yamaguchi et al., 2006; Iemmi et al., 2005; Nomura et al., 2006). Procedure (2) is also predictably effective for spherical mirror measurement to obtain higher-accuracy data. In this research, we have developed PS/PDI with two optical fibers point sources for absolute surface figure measurement of largeaperture optics. The inevitable wavefront distortion in principle is attempted to be eliminated through an inverse-problem-based numerical reconstruction of the wave, and highly precise measurements of spherical and aspherical mirrors has been realized. Section 2 provides a brief review of PDI research and PS methods. In section 3, a PS/PDI system for large-aperture optics is introduced and the interferogram recording procedure is explained. Section 4 describes the origin of measurement error estimated by interference between diffraction waves from two optical fibers. Effectivity of numerical wavefront restoration is described in section 5. In section 6, typical results of a spherical concave mirror are shown and the measurement accuracy is discussed. To demonstrate aspherical surface measurement, a highly distorted spherical concave mirror is measured and the result is discussed in section 7. An off-axis parabolic mirror surface and Si wafer surface are also measured and discussed in section 7. Measurement error of the PS/PDI for aspherical surfaces is studied. This chapter is concluded in section 8 .

\section{Principle of PS/PDI}

\subsection{Sphericity of diffraction wavefront}

A point diffraction interferometer (PDI) was developed by Smartt in the 1970s (Smartt \& Strong, 1972). The precursor to the PDI was an interferometer with an optical diffraction wave emitted from a tiny aperture fabricated on a so-called PDI plate, and the diffraction wave was used as a reference spherical wave for interferometry (Smartt \& Steel, 1975; 
Sommargren, 1996; Medecki et al., 1996; Millerd et al., 2004; Otaki et al., 2002b; Ota et al, 2001). Presently, demand is growing for highly precise optical surfaces, both spherical and aspherical, in the case of mirrors. In this section, (1) the principle of PDI measurement and (2) a phase-shift algorithm (de Groot, 1995a; de Groot, 1995b) for numerical processing of interferogram data are explained. In ordinary optical interferometry, surface distortion between the reference and test surfaces is measured and the measurement accuracy is limited by the accuracy of the substantial reference surface. In the PDI measurement, the reference surface is a diffraction wave emitted from a small aperture. Accordingly, wavefront error of the diffraction wave limits the PDI measurement accuracy. Numerical estimation of the wavefront error can be completed using diffraction theory. The broad theoretical base is the Huygens principle and the Helmholtz-Kirchhoff integral theorem is the strict definition. Moreover, the Fresnel-Kirchhoff diffraction integral is derived from application of the Helmholtz-Kirchhoff integral theorem to a tiny aperture and provides information regarding the diffraction wavefront precision (Born \& Wolf, 1959). As an example, fig. 1 shows the angular distribution of optical intensity and phase distortion of a diffraction wave emitted from a single-mode (SM) optical fiber core $4 \mu \mathrm{m}$ in diameter at a wavelength of $632.8 \mathrm{~nm}$. The resulting sphericity of the diffraction wave is less than $10^{-5} \lambda$ within an NA of 0.2. This means that a spherical concave mirror with a $500-\mathrm{mm}$ diameter and a 1500-mm radius of curvature (ROC) can be measured at an accuracy of less than $6 \mathrm{pm}$.

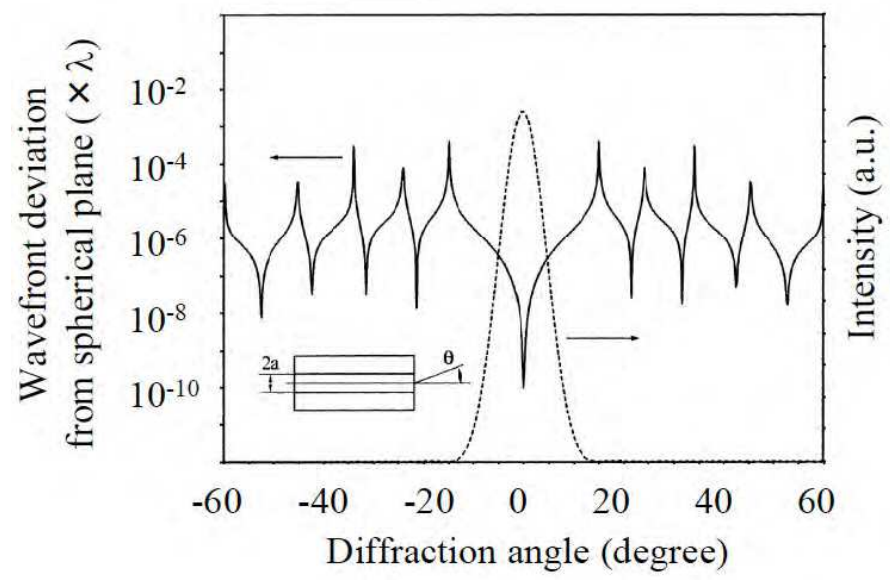

Fig. 1. Intensity and phase distribution of diffraction wavefront from tiny aperture.

\subsection{Wavefront and surface figure measurement by PDI}

With the highly precise diffraction wavefront, vertical spatial resolution of the PDI measurement of the sub-nanometer order, and wavefront estimation using a PDI plate and surface figure measurement with an optical fiber or tiny pinhole are already realized. Fig. 2 explains the principle of PDI measurement using a PDI plate. This configuration is used to evaluate wavefront error in imaging optics. A focused light wave makes an Airy pattern according to the diffraction theory. In the Airy disk, more than $90 \%$ of the light wave is converged. The PDI plate is then placed at the focal point. The plate has two apertures; one (hole a) is for the Airy disk and the other (hole b) is for generation of a reference diffraction 


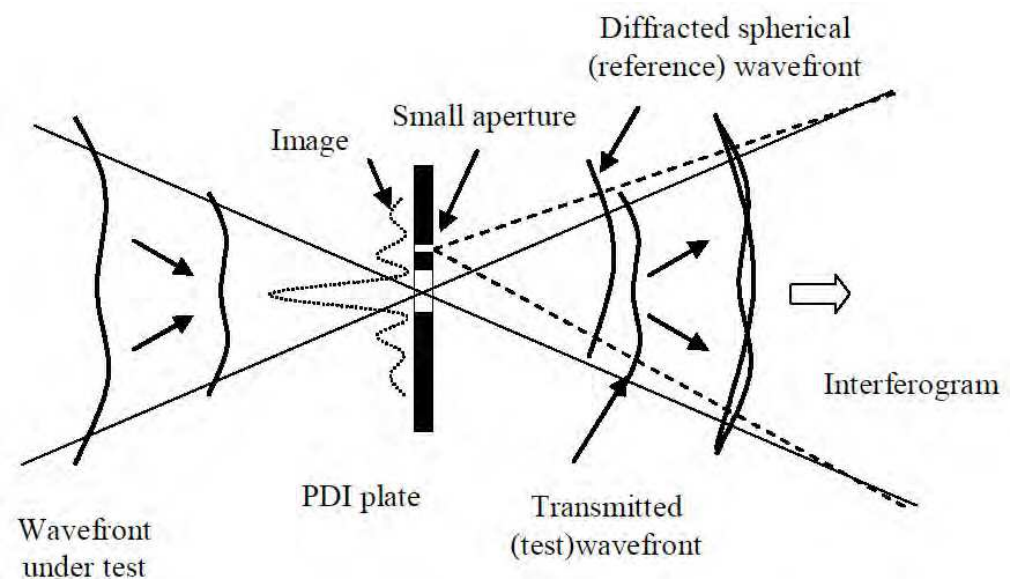

Fig. 2. PDI configuration with PDI plate.

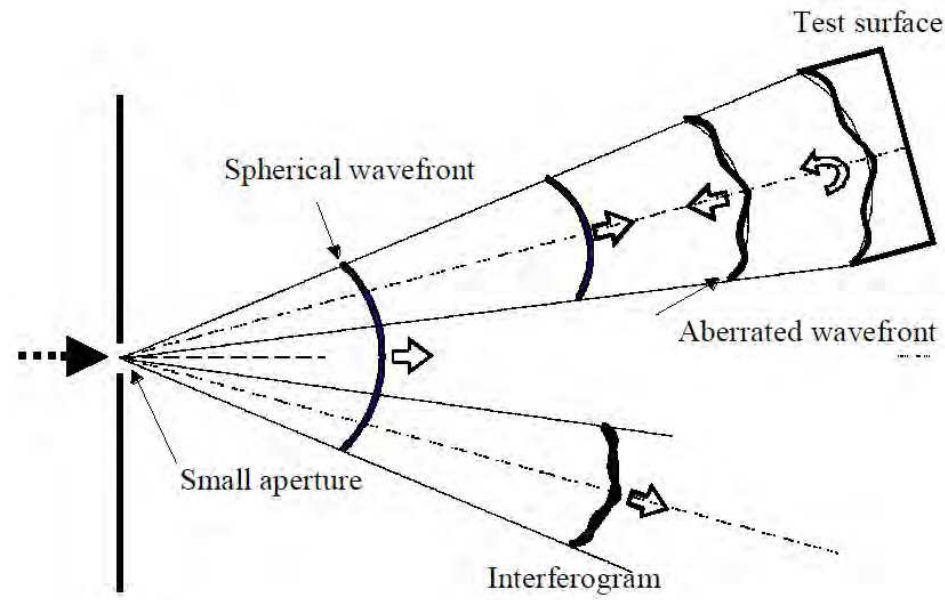

Fig. 3. PDI configuration with a tiny pinhole.

wave. Hole $b$ is located on the first ring pattern of the Airy disk, and the resultant diffraction wave does not include information about the test wave. Hole a gives passage to a component inside the Airy disk. As a result, the wavefront emitted from hole a interferes with the reference wavefront emitted from hole $b$ and optical interference can be accomplished. Fig. 3 shows the PDI configuration for surface figure measurement with a single tiny aperture (Sommargren, 1996). A pinhole or single-mode optical fiber is accepted as the aperture. Part of the diffraction wave generated at the aperture makes a complete trip to the test surface and is reflected around the aperture. The reflected test wave interferes with the rest of the diffraction wave, serving as a reference wave, emitted from the tiny aperture. The resultant interferogram provides surface figure data of the test mirror. However, this configuration has several disadvantages. Reflection around the aperture 
could distort the test wavefront because of surface roughness or the lack of a reflecting surface. This configuration could not be applied to large test optics because a single diffraction wavefront is divided into a test wave and a reference wave.

\subsection{Analysis of interferogram by phase-shift method}

Optical interferometry treats a series of interferograms for precise measurement. Phase-shift algorithms are of the accurate procedures for interferograms. Fig. 4 shows the principle of the phase-shift procedure. Determining a step-by-step phase difference $\varphi(t)$ between reference and test surfaces causes sinusoidal changes in optical intensity at point $P$. A set of discrete data is diagnosed to obtain the initial phase value at point P. Many algorithms exist that determine the initial phase value and the standard procedure is based on Fourier transformation (Freischlad \& Koliopoulos, 1990; Larkin \& Oreb, 1992). In the following section, the entire experimental dataset is processed using seven bucket algorithms.

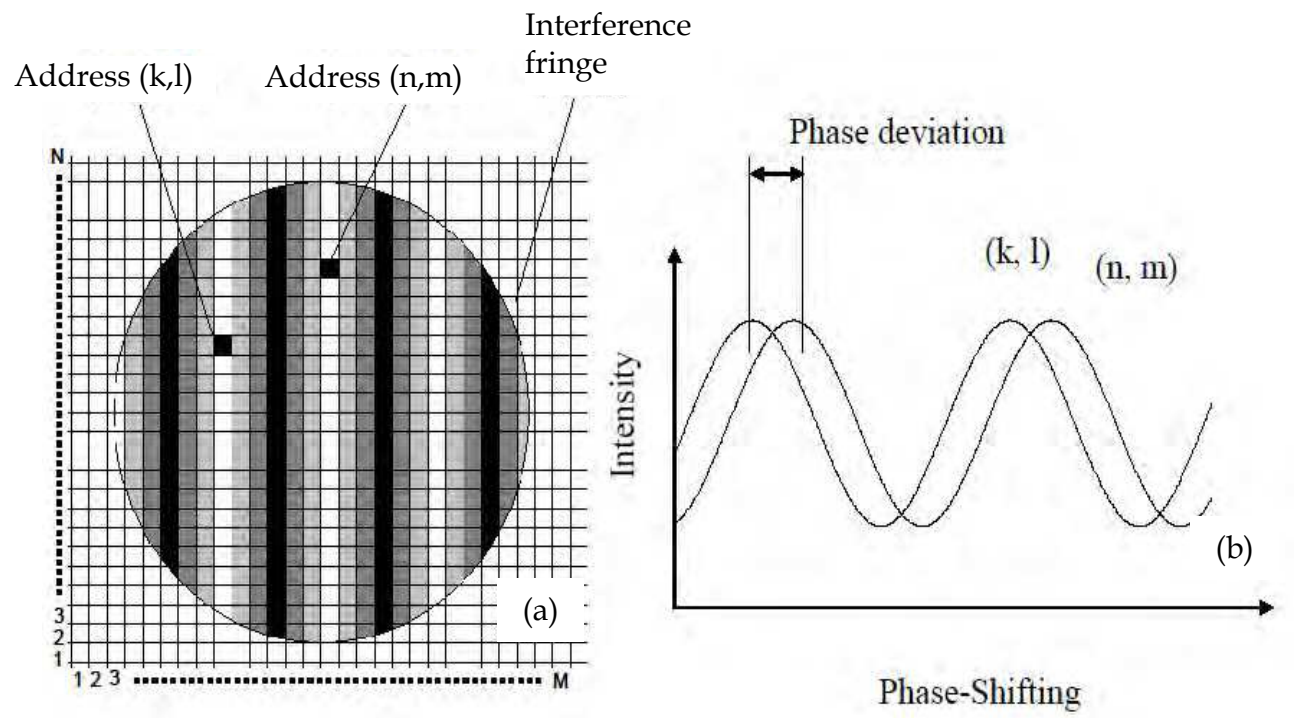

Fig. 4. Principle of phase shift method. (1) CCD pixels and interference pattern, (2) intensity changes in the pixels by phase shift interferometry.

\section{PDI Configuration with two optical fibers for large-aperture optics}

\subsection{Experimental apparatus}

In this section, detailed optical configuration of a PS/PDI system with two optical fibers serving as tiny apertures is described. The recording process for phase-shifting interferograms is also explained. Fig. 5 shows the principle of the PS/PDI configuration with two tiny apertures. A light wave with sufficiently long coherence length is divided into two waves and each wave is introduced into a small aperture. Two independent spherical diffraction waves are then generated. One wave propagates to the test surface and the other serves as the reference wave and propagates in the opposite direction. Therefore, the whole wavefront can be used for surface figure measurement. In the entire optical path, there are 


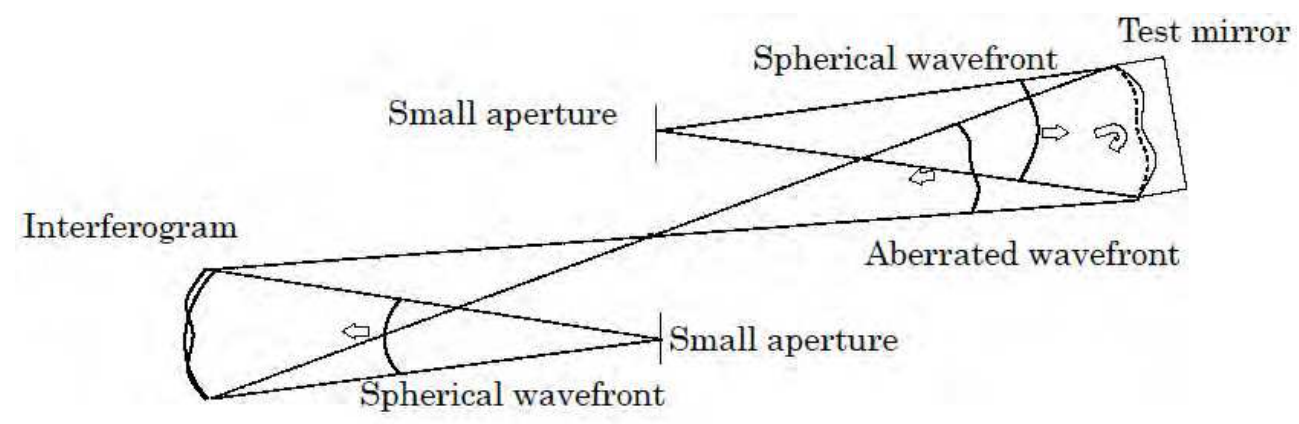

Fig. 5. Optical configuration of PDI with two small apertures.

no additional optics and, in principle, there is no disturbance in the propagating waves. This PDI configuration makes full use of the ultra-high-accurate wavefront as reference. However, this is not a complete common-path system and tilt fringe inevitably occurs due to spacing between the focal point of the test wave (point a) and the origin of the reference wave (point $b$ ). Dense tilt fringes are not recorded correctly by a charge-coupled device (CCD) sensor because the width of each fringe is smaller than or comparable to the size of a CCD pixel. To avoid such a consequence, a shorter distance between points a and $b$ and a correction process are needed. A schematic diagram of an actual PS/PDI system is shown in fig. 6 as a means for realizing the above discussed outcome. For temporal stability of the system, the entire optical system is installed on an air-suspension-type vibration-isolation table in a temperature-controlled room. Air circulation in the experiment room causes optical path differences through inhomogeneous refractive index of the atmosphere, resulting in random error in the experimental result. This error is suppressed by a large styrofoam box serving as both an air screen and an adiabatic case. A He-Ne laser light source, which also serves as a heat source, is installed outside the box. The He-Ne laser is linearly polarized and its wavelength is $632.8 \mathrm{~nm}$. In this optical system, light from the components is blocked from the laser using an optical isolator $(60 \mathrm{~dB})$ and the laser output power is stabilized. A half-wave plate (HWP) is used to rotate the light polarization to adjust the branching ratio of the laser beam at the polarization beam splitter (PBS). Each branched beam is introduced into the core of an SM optical fiber of $4-\mu \mathrm{m}$ diameter. Diagnostic and reference waves are thus generated independently. Changing the branching ratio at the PBS optimizes the interferogram contrast generated on the CCD camera. The interferogram is recorded as a 14-bit digital image by the CCD camera with an electronic shutter. In the interferometry system, the phase-shift method is executed by changing the optical path of the diagnostic wave. The aluminum mirror on the single-axis PZT stage in fig. 6 is translated along the optical path and the step-by-step motion is synchronized with the CCD camera shutter. Thus, a series of phase-shifting interferograms are obtained. In this PDI system, separation between the close fiber ends shown in fig. 7 causes a partial diffraction wave due to the opposed fiber end. Therefore, the fiber end is obliquely polished with a pipette grinder to bend the beam output axis away from the fiber axis. Fig. 8 shows the relation between the angle of polish and the output beam direction. For optimal installation configuration, the fiber ends are obliquely polished at 15 degrees from surface normal to fiber axis. As a result, the fiber end serving as the diffraction wave origin has an ellipsoidal section, which may degrade the accuracy of the spherical wavefront. However, as shown in fig. 9, the diffraction wavefront caused by the ellipsoidal fiber core still generates a 


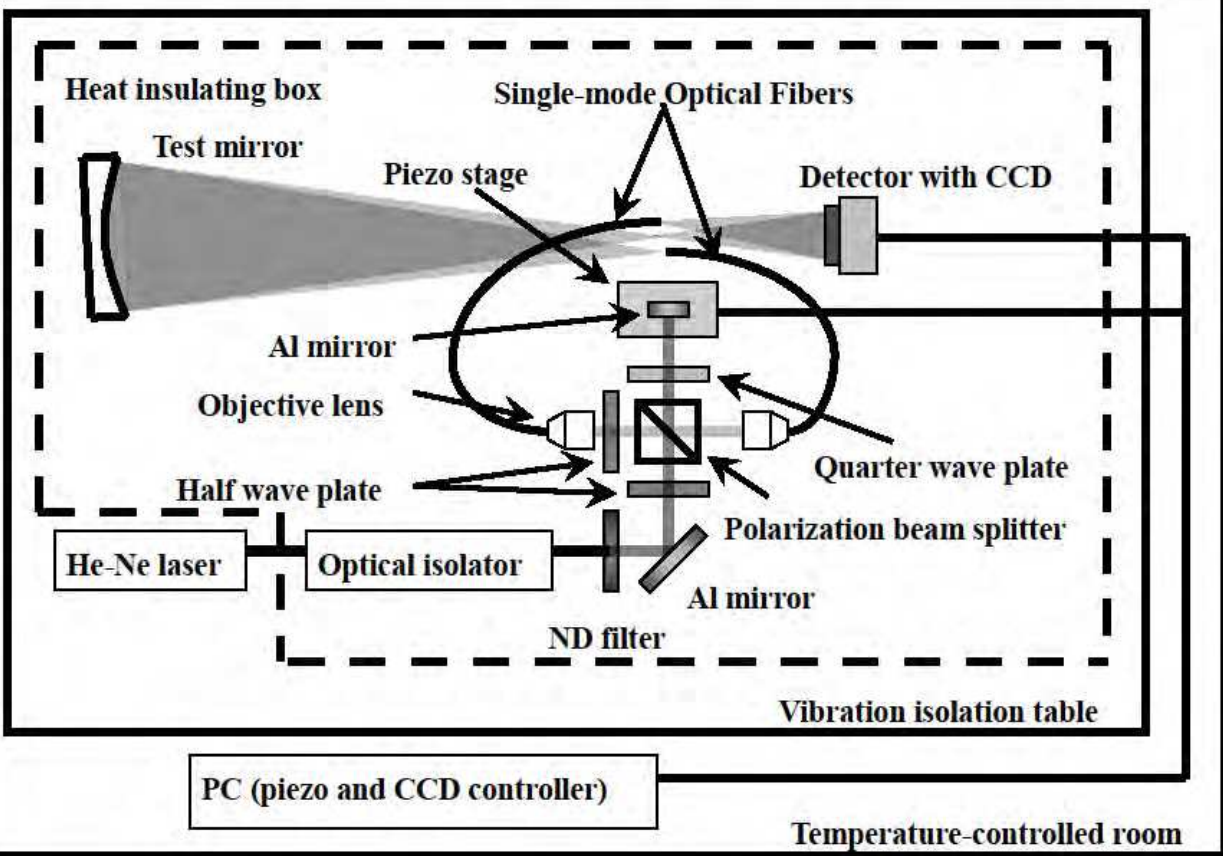

Fig. 6. Schematic diagram of PS/PDI system with two point sources of single mode optical fibers.

microscopic photo of a sharpened and obliquely polished fiber end is shown in fig. 12. The polished section must include both the core and surrounding clad area.

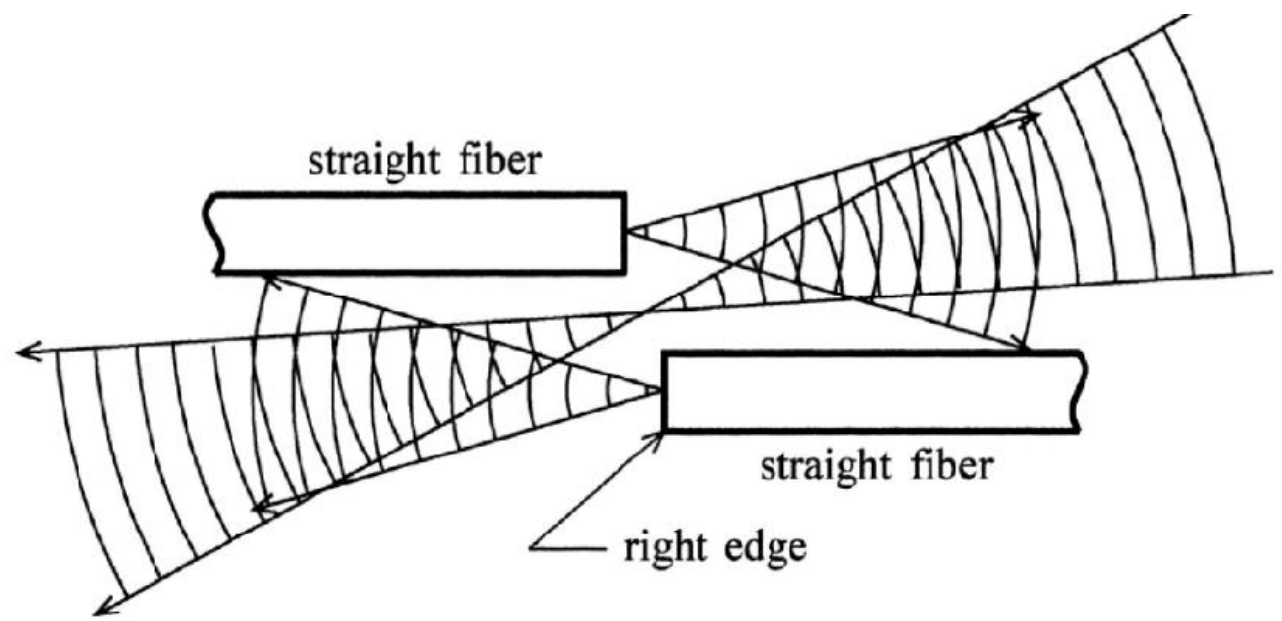

Fig. 7. Two fiber ends arranged in opposite directions and the outgoing diffraction waves. 


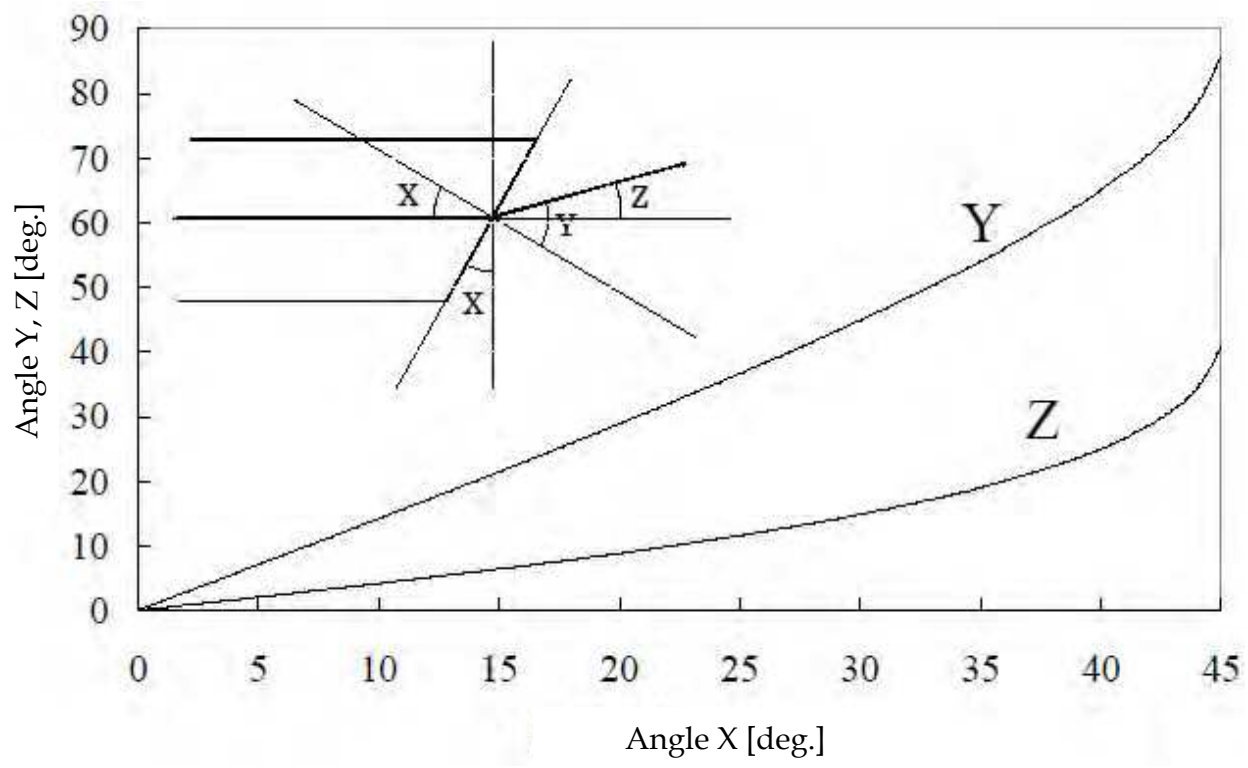

Fig. 8. Change in direction of the outgoing diffraction wave by obliquely polished angle.

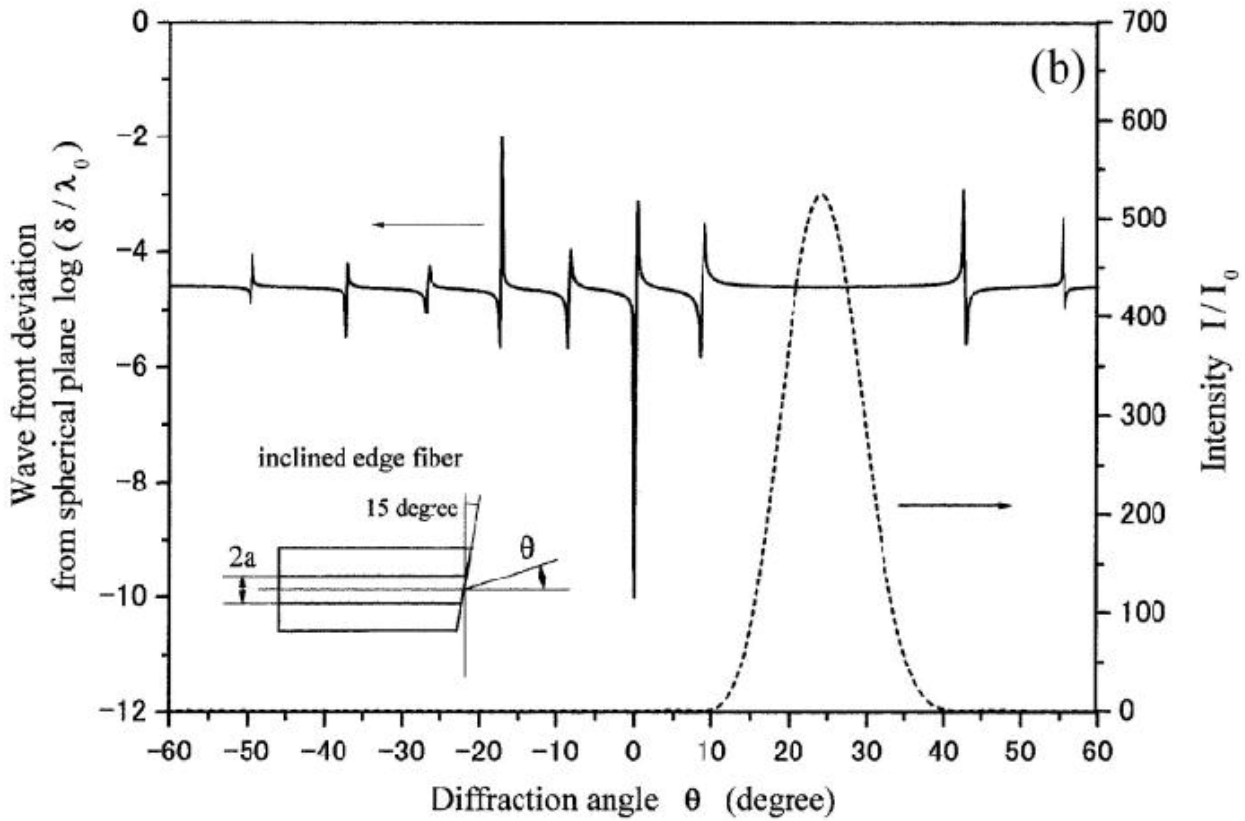

Fig. 9. Intensity and phase distribution of diffraction wavefront emitted from obliquely polished fiber end. 


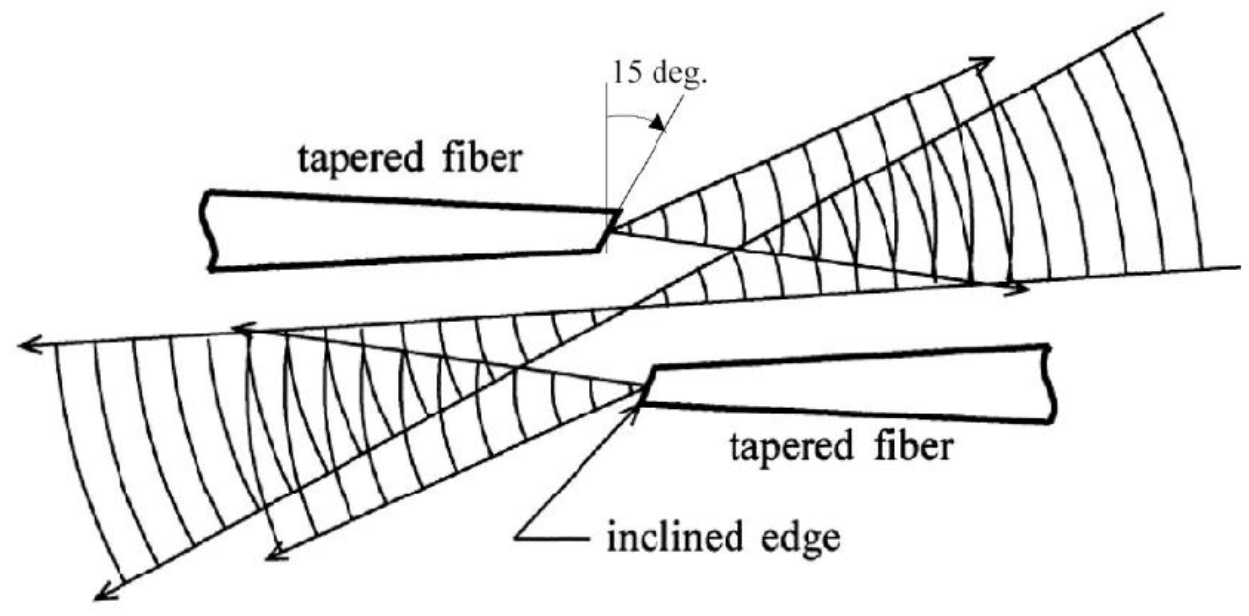

Fig. 10. Resultant configuration of two fiber ends having sharpened apex and obliquely polished ends.

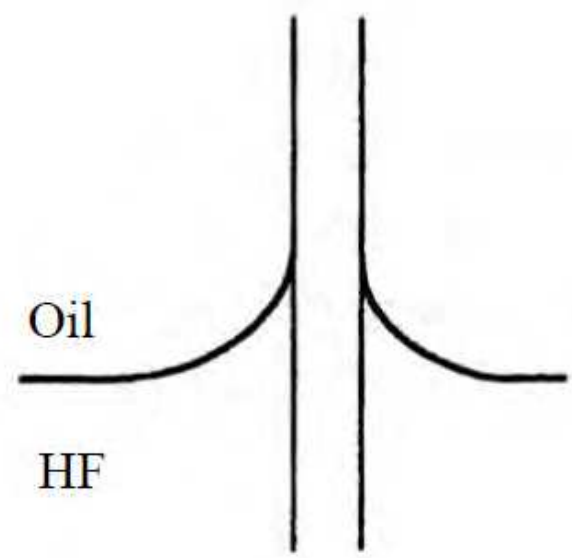

(a) Immersion



(b) Etch

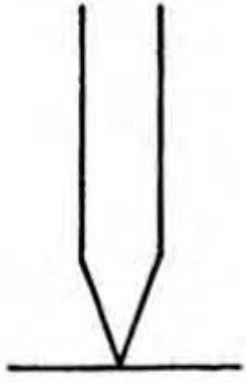

(c) Self-Stop

Fig. 11. Chemical etching of fiber end at boundary between oil and conc. HF. 


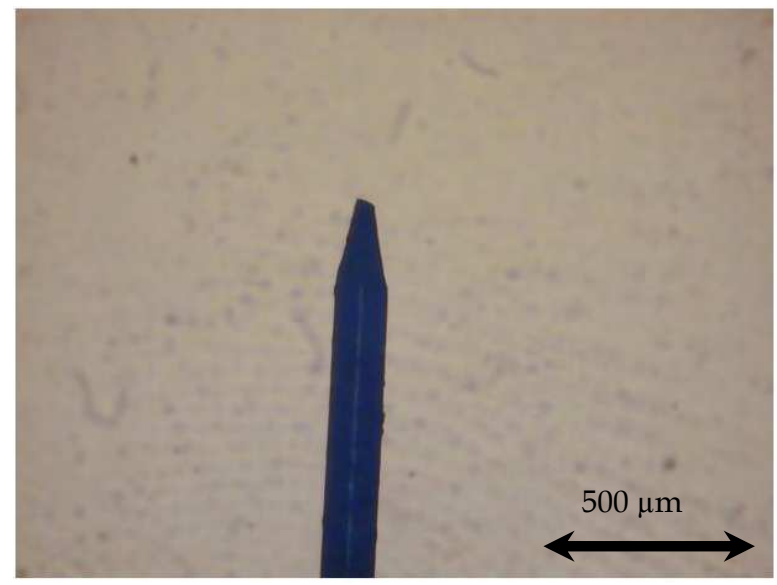

Fig. 12. Microscopic photo of single-mode optical fiber end after chemical etching.

highly spherical wave. Because the fiber end is close to the focal point of the reflected diagnostic wave, the fiber end must have a sharpened tip. With the sharpened fibers, optical configuration as shown in fig. 10 will be accomplished and the whole diffraction wavefront will be used for large-aperture optics. The sharpened end of the fiber is formed with a mixture of hydrofluoric acid and organic fluids. At the interface between the two liquids, the fiber end is automatically sharpened owing to surface tension as shown in fig. 11. A

\subsection{Recording of interferograms}

For precise data analysis using the phase-shift method, serial interferograms having an equivalent phase difference must be recorded. The CCD shutter and movement of the Al mirror or PZT stage are computer controlled using LabVIEW. Motion of the PZT stage is optimized to restrict mechanical vibration of the mirror. Fig. 13 shows the control system and fig. 14 shows a flow chart of the measurement.

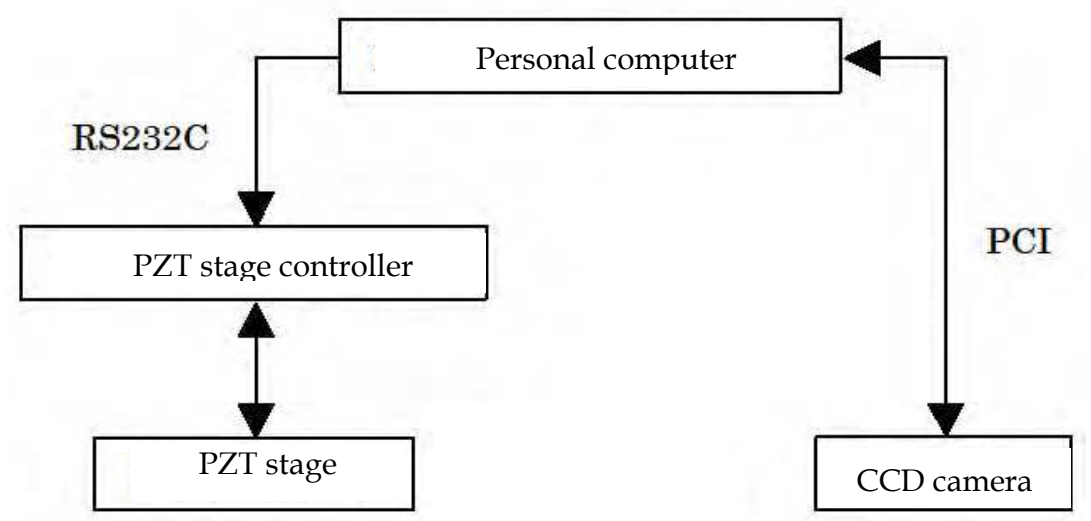

Fig. 13. Diagram for control of the PS/PDI system. 




Fig. 14. Flow chart for the PS/PDI measurement. 


\section{Estimation of measurement accuracy by null test}

\subsection{Wavefront analysis procedure}

Traditional PS/PDIs analyze interferograms caused by a diagnostic wavefront reflected by the test surface and a reference wavefront to obtain the surface figure of the test mirror (Smartt \& Steel, 1975; Sommargren, 1996; Medecki et al., 1996; Millerd et al., 2004; Otaki et al., 2002b). In contrast, the PS/PDI described in this chapter uses two optical fibers as light sources, and the interferogram generated by these two fibers is recorded by a CCD camera. PS analysis of this interferogram provides the accuracy of the spherical wavefronts or measurement error of the PS/PDI system. Two optical-fiber point sources cannot be physically overlapped. As a result, an interferogram having many black-and-white-banded stripes is seen on the screen or CCD surface (Born \& Wolf, 1959). We assume an optical system as shown in fig. 15. Points A and B are fiber ends for reference and test waves, respectively, and they are origin symmetrical. Projection plane $S$, which has a tilt component, is described in the following equation.

$$
z=\alpha x+\beta y+d
$$

An optical path difference between $\mathrm{AP}$ and $\mathrm{BP}$ at a point $\mathrm{P}$ on $\mathrm{S}$ can be written as follows:

$$
\begin{aligned}
\delta_{\mathrm{i}} & =\overline{\mathrm{AP}}-\overline{\mathrm{BP}} \\
& =\sqrt{\left(x_{0}-x_{\mathrm{i}}\right)^{2}+\left(y_{0}-y_{\mathrm{i}}\right)^{2}+\left(z_{0}-z_{\mathrm{i}}\right)^{2}}-\sqrt{\left(-x_{0}-x_{\mathrm{i}}\right)^{2}+\left(-y_{0}-y_{\mathrm{i}}\right)^{2}+\left(-z_{0}-z_{\mathrm{i}}\right)^{2}}
\end{aligned}
$$

Here, $\mathrm{P}$ is the $\mathrm{i}$-th pixel on the CCD detector. When the path difference is an even multiple of the half-wavelength of the light, interference fringe becomes bright, and an odd multiple of the half-wavelength causes dark fringe. As an example, numerical simulation of the fringe pattern is shown in fig. 16. In this figure, fiber-to-fiber distance is $60 \mu \mathrm{m}$ and the projection screen is parallel to the $x y$ plane at $\mathrm{z}=150 \mathrm{~mm}$. This interference pattern is a contour having $\lambda / 2$ spacing, and an initial phase map will be calculated with this pattern through a phaseshift algorithm. Then, by changing the optical path difference at intervals of $\Pi / 2$, seven continuously changing interference patterns are created. The seven patterns are processed through seven bucket algorithms with a Von Hann window, as summarized in table 1. Consequently, an initial phase map is formed, as shown in fig. 17. In the phase-shift algorithm, a phase is calculated using an arctangent function and the map has many discontinuous lines, as can be seen in fig. 17. Here, we must unwrap the phase information. After unwrapping, a continuous phase map is generated, as can be seen in fig. 18. The initial phase map shown in fig. 18 indicates a phase difference between the reference wave from a point and the test wave from another point on the projection plane. To obtain the surface figure of test wave, location of both the reference fiber end and projection plane must be determined, and these position coordinates are analytically derived. Locations of every component in fig. 15 can be determined through the least-square method with the phase map on a projection screen because the test and reference waves are assumed to be ideal spherical waves. The optical path difference between AP and BP can be obtained from the schematic diagram of the optics, $\delta_{i}$, and measurement result, $\Delta_{i}$. Locations of points $A, B$, and the projection screen are determined by minimizing $\mathrm{s}$, the square sum of the differences between $\delta_{i}$ and $\Delta_{i}$. Here, the measurement result is a relative phase map and the value is described by the following equation with parameter $h$. 


\begin{tabular}{|c|c|c|c|c|}
\hline$P$ & $P^{i}$ & $a$ & $x$ & $\tau$ \\
\hline 5 & b & $\pi / 2$ & 2 & $\frac{2\left(s_{2}-g_{1}\right)}{-\left(g_{1}+s_{3}\right)+2 b_{3}}$ \\
\hline 6 & 8 & $\pi / 2$ & 6 & $\frac{-\left(g_{0}-g_{B}\right)-3\left(g_{1}+E_{4}\right)+4\left(g_{2}-g_{3}\right)}{\left.-\left(G_{0}\right)+E_{0}\right)+3\left(E_{1}-B_{3}\right)+4\left(g_{2}+g_{2}\right)}$ \\
\hline 7 & 8 & $\pi / 2$ & 8 & $\frac{-\left(B_{0}-g_{6}\right)+7\left(g_{2}-B_{4}\right)}{-4\left(E_{1}+B_{5}\right)+8 g_{0}}$ \\
\hline 11 & 12 & $\pi / 2$ & 16 & $\frac{\left.\left(g_{0}-g_{10}\right)-g_{(g}-g_{8}\right)+15\left(g_{4}-g_{0}\right)}{4\left(g_{6}+g_{0}\right)-12\left(g_{2}+g_{7}\right)+16 g_{5}}$ \\
\hline-8 & 9 & $2 \pi / 3$ & 20 & $\frac{2\left(g_{0}-g_{7}\right)-7\left(g_{1}-g_{a}\right)+17\left(g_{0}-g_{0}\right)}{\left(g_{0}+g_{7}\right)+4\left(g_{1}+g_{6}\right)-15\left(g_{2}+g_{3}\right)+10\left(g_{1}+g_{4}\right)}$ \\
\hline 8 & 12 & $\pi / 3$ & 2.7 & $\frac{-g_{0}+g_{1}+g_{9}-g_{1}-g_{0}+g_{7}+2\left[g_{2}-g_{6}\right)}{-\left(g_{0}+g_{1}+g_{6}+g_{7}\right)+2\left(g_{2}+g_{0}\right)}$ \\
\hline 9 & ${ }_{16}$ & $\pi / 4$ & 2 & $\frac{g_{1}+g_{3}-g_{s}-g_{1}+2\left(R_{2}-g_{6}\right)}{-g_{0}-g_{1}+E_{3}+R_{3}-g_{3}-g_{s}+2 g_{4}}$ \\
\hline
\end{tabular}

Table 1. Examples of phase shift algorithms based on Von Hann window. Equation T at $P=7$ is adopted in this study.

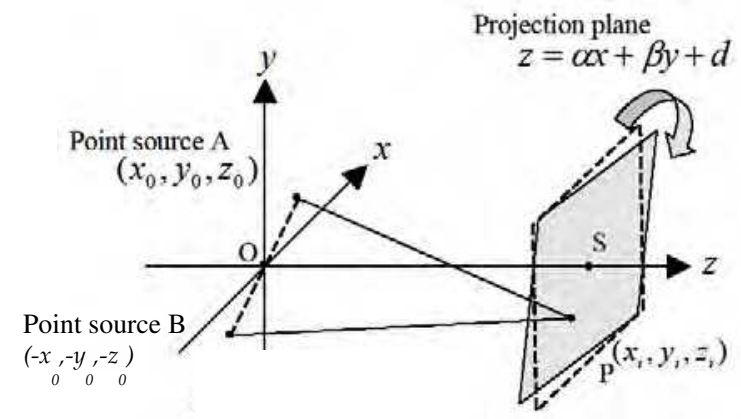

Fig. 15. Coordinates for wave front procedure.



Fig. 16. Computer generated interference pattern. 


$$
s=\sum_{i}\left\{\left(\Delta_{i}-h\right)-\delta_{i}\right\}^{2}
$$

As a result, the seven parameters $x_{0}, y_{0}, z_{0}, a, \beta, d$, and $h$ are used to determine the locations of the two fiber ends, projection screen, and the offset of phase map. A small change of $\delta_{i}$ by changing $x_{0}, y_{0}, z_{0}, a, \beta$, and $d$ can be expressed as follows.

$$
\Delta \delta_{\mathrm{i}}=\frac{\partial \delta_{\mathrm{i}}}{\partial x_{0}} \Delta x_{0}+\frac{\partial \delta_{\mathrm{i}}}{\partial y_{0}} \Delta y_{0}+\frac{\partial \delta_{\mathrm{i}}}{\partial z_{0}} \Delta z_{0}+\frac{\partial \delta_{\mathrm{i}}}{\partial \alpha} \Delta \alpha+\frac{\partial \delta_{\mathrm{i}}}{\partial \beta} \Delta \beta+\frac{\partial \delta_{\mathrm{i}}}{\partial d} \Delta d
$$

Parameter s indicated in Eq. (3) is then defined as follows.

$$
s=\sum_{\mathrm{i}}\left[\left(\Delta_{\mathrm{i}}-h\right)-\left(\delta_{\mathrm{i}}+\Delta \delta_{\mathrm{i}}\right)\right]^{2}
$$

To minimize parameter s, the following condition is used to form simultaneous equations.

$$
\begin{aligned}
& \frac{\partial s}{\partial \Delta x_{0}}=0 \\
& \frac{\partial s}{\partial \Delta y_{0}}=0 \\
& \frac{\partial s}{\partial \Delta z_{0}}=0 \\
& \frac{\partial s}{\partial \Delta \alpha}=0 \\
& \frac{\partial s}{\partial \Delta \beta}=0 \\
& \frac{\partial s}{\partial \Delta d}=0 \\
& \frac{\partial s}{\partial h}=0
\end{aligned}
$$

Optical path differences AP and BP are geometrically determined as follows.

$$
\begin{aligned}
& \frac{\partial \delta_{\mathrm{i}}}{\partial x_{0}}=\frac{\left(x_{0}-x_{\mathrm{i}}\right)}{s_{1}}+\frac{\left(-x_{0}-x_{\mathrm{i}}\right)}{s_{2}} \\
& \frac{\partial \delta_{\mathrm{i}}}{\partial y_{0}}=\frac{\left(y_{0}-y_{\mathrm{i}}\right)}{s_{1}}+\frac{\left(-y_{0}-y_{\mathrm{i}}\right)}{s_{2}}
\end{aligned}
$$




$$
\begin{aligned}
& \frac{\partial \delta_{\mathrm{i}}}{\partial z_{0}}=\frac{\left(z_{0}-\alpha x_{\mathrm{i}}-\beta y_{\mathrm{i}}-d\right)}{s_{1}}+\frac{\left(-z_{0}-\alpha x_{\mathrm{i}}-\beta y_{\mathrm{i}}-d\right)}{s_{2}} \\
& \frac{\partial \delta_{\mathrm{i}}}{\partial \alpha}=\frac{x_{\mathrm{i}}\left(z_{0}-\alpha x_{\mathrm{i}}-\beta y_{\mathrm{i}}-d\right)}{s_{1}}-\frac{x_{\mathrm{i}}\left(-z_{0}-\alpha x_{\mathrm{i}}-\beta y_{\mathrm{i}}-d\right)}{s_{2}} \\
& \frac{\partial \delta_{\mathrm{i}}}{\partial \beta}=\frac{y_{\mathrm{i}}\left(z_{0}-\alpha x_{\mathrm{i}}-\beta y_{\mathrm{i}}-d\right)}{s_{1}}-\frac{y_{\mathrm{i}}\left(-z_{0}-\alpha x_{\mathrm{i}}-\beta y_{\mathrm{i}}-d\right)}{s_{2}} \\
& \frac{\partial \delta_{\mathrm{i}}}{\partial d}=\frac{\left(z_{0}-\alpha x_{\mathrm{i}}-\beta y_{\mathrm{i}}-d\right)}{s_{1}}-\frac{\left(-z_{0}-\alpha x_{\mathrm{i}}-\beta y_{\mathrm{i}}-d\right)}{s_{2}}
\end{aligned}
$$

With these equations, each term in Eq. (4) can be described as follows.

$$
\begin{aligned}
& \frac{\partial s}{\partial \Delta x_{0}}=2 \sum_{\mathrm{i}}\left(-\frac{\partial \delta_{\mathrm{i}}}{\partial x_{0}}\right)\left[\Delta_{\mathrm{i}}-h-\left(\delta_{\mathrm{i}}-\Delta \delta_{\mathrm{i}}\right)\right]=0 \\
& \frac{\partial s}{\partial \Delta y_{0}}=2 \sum_{\mathrm{i}}\left(-\frac{\partial \delta_{\mathrm{i}}}{\partial y_{0}}\right)\left[\Delta_{\mathrm{i}}-h-\left(\delta_{\mathrm{i}}-\Delta \delta_{\mathrm{i}}\right)\right]=0 \\
& \frac{\partial s}{\partial \Delta z_{0}}=2 \sum_{\mathrm{i}}\left(-\frac{\partial \delta_{\mathrm{i}}}{\partial z_{0}}\right)\left[\Delta_{\mathrm{i}}-h-\left(\delta_{\mathrm{i}}-\Delta \delta_{\mathrm{i}}\right)\right]=0 \\
& \frac{\partial s}{\partial \Delta \alpha}=2 \sum_{\mathrm{i}}\left(-\frac{\partial \delta_{\mathrm{i}}}{\partial \alpha}\right)\left[\Delta_{\mathrm{i}}-h-\left(\delta_{\mathrm{i}}-\Delta \delta_{\mathrm{i}}\right)\right]=0 \\
& \frac{\partial s}{\partial \Delta \beta}=2 \sum_{\mathrm{i}}\left(-\frac{\partial \delta_{\mathrm{i}}}{\partial \beta}\right)\left[\Delta_{\mathrm{i}}-h-\left(\delta_{\mathrm{i}}-\Delta \delta_{\mathrm{i}}\right)\right]=0 \\
& \frac{\partial s}{\partial \Delta d}=2 \sum_{\mathrm{i}}\left(-\frac{\partial \delta_{\mathrm{i}}}{\partial d}\right)\left[\Delta_{\mathrm{i}}-h-\left(\delta_{\mathrm{i}}-\Delta \delta_{\mathrm{i}}\right)\right]=0 \\
& \frac{\partial s}{\partial h}=2 \sum_{\mathrm{i}}\left[\Delta_{\mathrm{i}}-h-\left(\delta_{\mathrm{i}}-\Delta \delta_{\mathrm{i}}\right)\right]=0
\end{aligned}
$$

As a result, parameter $\mathrm{s}$ is determined by six small changes, $\Delta \mathrm{x}_{0}, \Delta \mathrm{y}_{0}, \Delta \mathrm{z}_{0}, \Delta \mathrm{a}, \Delta \beta$, and $\Delta \mathrm{d}$. Additionally, from Eq. (5) and (6), the following expressions are formed.

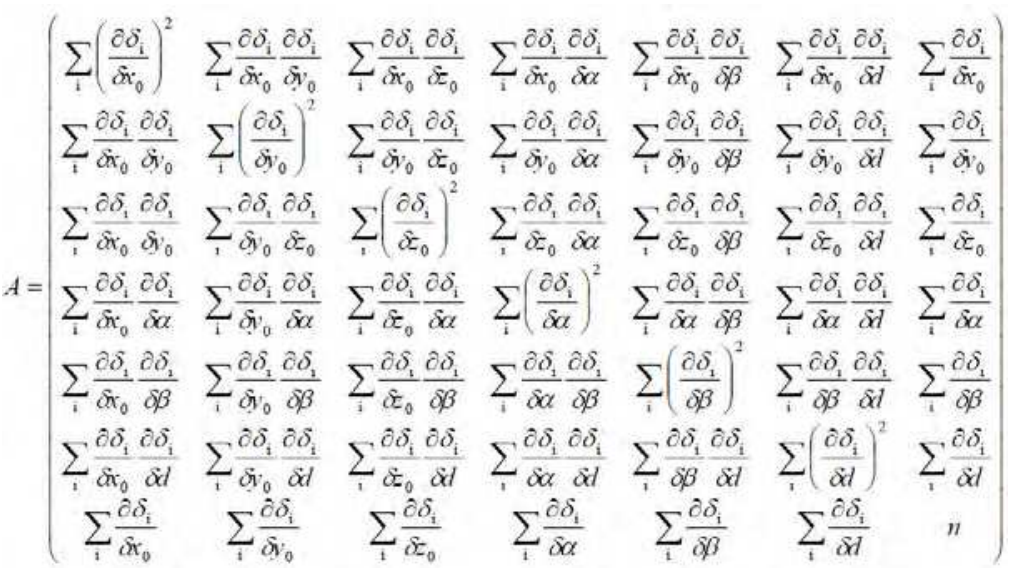

The resultant simultaneous equations are described as follows. 


$$
B=\left(\begin{array}{l}
\sum_{\mathrm{i}} \frac{\partial \delta_{\mathrm{i}}}{\partial x_{0}}\left(\Delta_{\mathrm{i}}-\delta_{\mathrm{i}}\right) \\
\sum_{\mathrm{i}} \frac{\partial \delta_{\mathrm{i}}}{\partial y_{0}}\left(\Delta_{\mathrm{i}}-\delta_{\mathrm{i}}\right) \\
\sum_{\mathrm{i}} \frac{\partial \delta_{\mathrm{i}}}{\partial z_{0}}\left(\Delta_{\mathrm{i}}-\delta_{\mathrm{i}}\right) \\
\sum_{\mathrm{i}} \frac{\partial \delta_{\mathrm{i}}}{\partial \alpha}\left(\Delta_{\mathrm{i}}-\delta_{\mathrm{i}}\right) \\
\sum_{\mathrm{i}} \frac{\partial \delta_{\mathrm{i}}}{\partial \beta}\left(\Delta_{\mathrm{i}}-\delta_{\mathrm{i}}\right) \\
\sum_{\mathrm{i}} \frac{\partial \delta_{\mathrm{i}}}{\partial d}\left(\Delta_{\mathrm{i}}-\delta_{\mathrm{i}}\right) \\
\sum_{\mathrm{i}}\left(\Delta_{\mathrm{i}}-\delta_{\mathrm{i}}\right)
\end{array}\right) \quad X=\left(\begin{array}{l}
\Delta x_{0} \\
\Delta y_{0} \\
\Delta z_{0} \\
\Delta \alpha \\
\Delta \beta \\
\Delta d \\
h
\end{array}\right) \quad A X=B
$$

Solution to the system of equations gives $\Delta \mathrm{x}_{0}, \Delta \mathrm{y}_{0}, \Delta \mathrm{z}_{0}, \Delta \mathrm{a}, \Delta \beta$, and $\Delta \mathrm{d}$, and all of the locations for components in fig. 15 will be determined. Using the above procedures and the data shown in fig. 18, the following values are obtained: $x=29.9 \mu \mathrm{m}, \mathrm{y}=0.0 \mu \mathrm{m}, \mathrm{z}=0.2 \mu \mathrm{m}$, $\mathrm{d}=150.0 \mathrm{~mm}, \mathrm{a}=0.0$, and $\beta=0.0$, and the surface figure deviation of the test surface from an ideal sphere is determined as shown in fig. 19, which is highly spherical. The $\mathrm{x}$ and $\mathrm{z}$ values are slightly different from the initial values due to computational error.

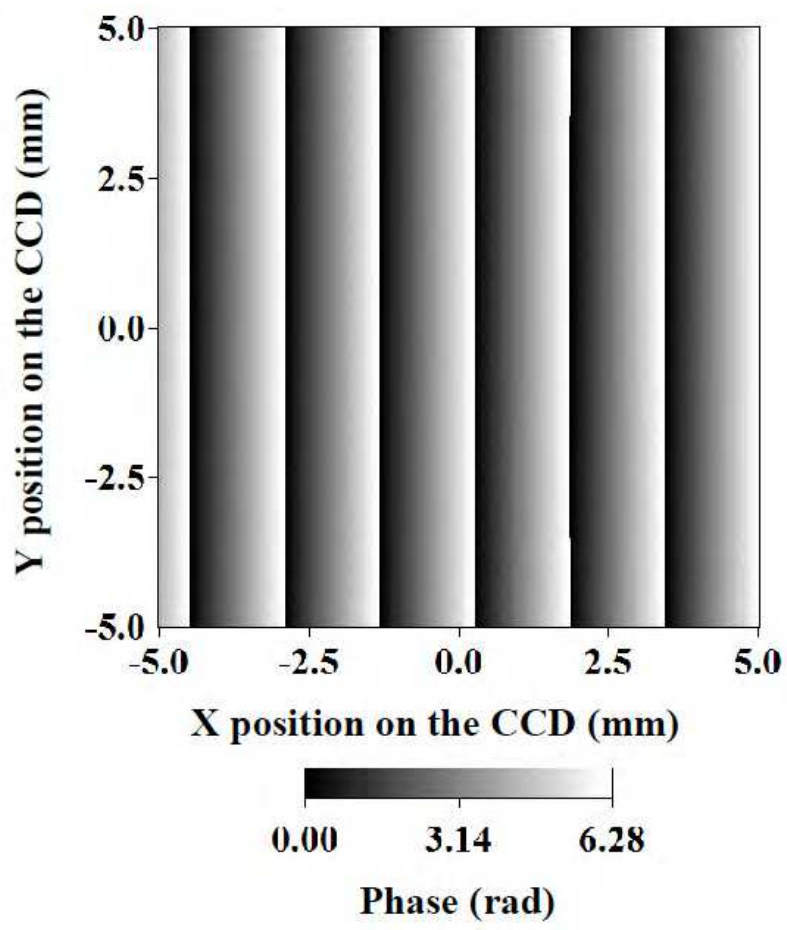

Fig. 17. Wrapped initial phase map with discontinuity. 


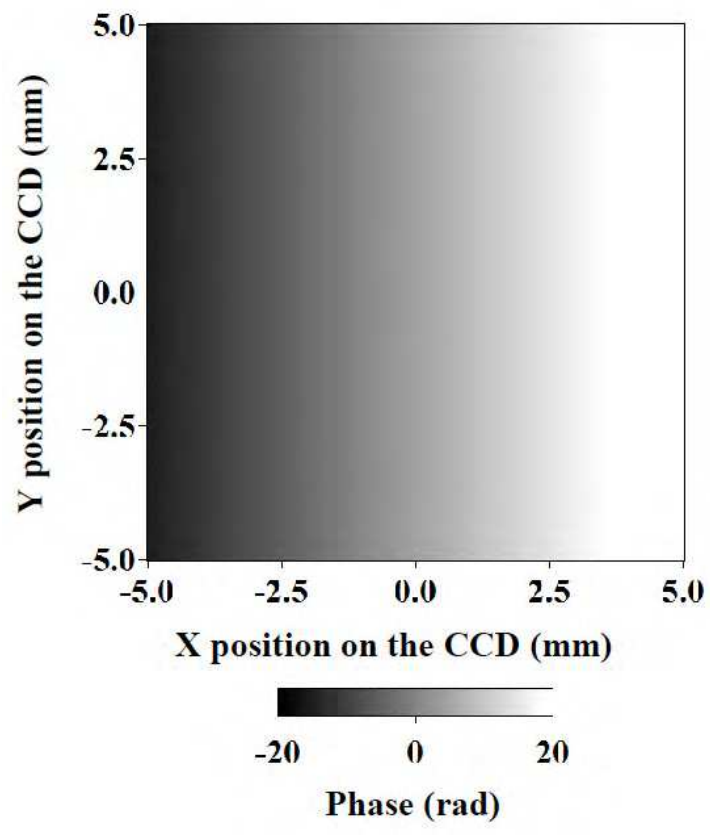

Fig. 18. Unwrapped continuous initial phase map.

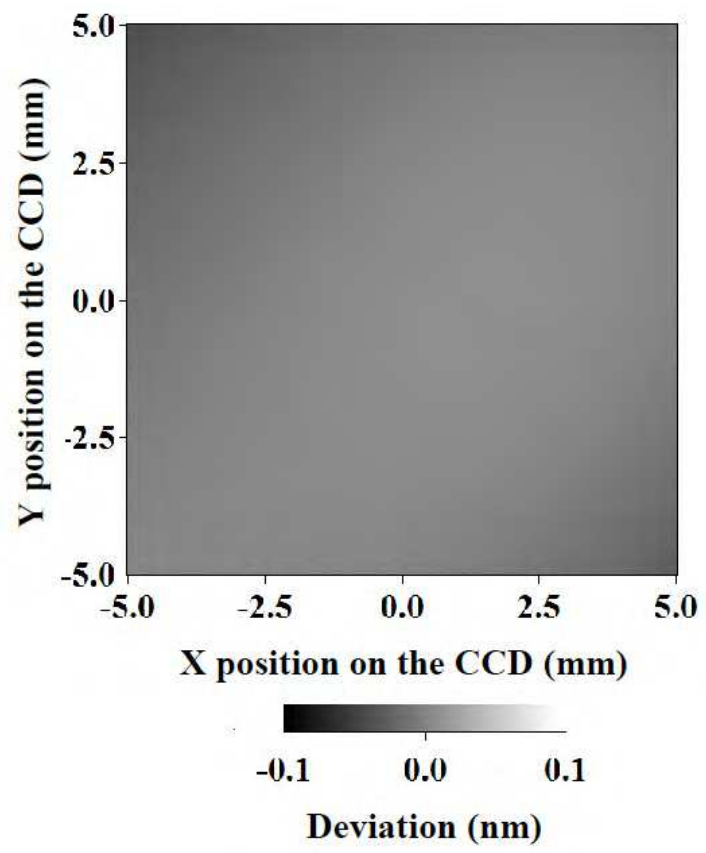

Fig. 19. Deviation of test wavefront from ideal spherical surface. 


\subsection{Measurement error caused by projection plane}

At the beginning of error estimation, the interference fringe pattern was recorded using a CCD detector through an imaging lens as shown in fig. 20. However, the two diffraction waves from the fiber ends pass through different parts of the lens and receive different amounts of aberrations. As an example, error caused by a commercial plane-convex lens is estimated by the ray-trace method. The convex lens surface is not hyperboloidal but spherical, and a type of aberration must be included on the diffraction waves. The optical path differences with and without the imaging lens are estimated as shown in fig. 21. This phenomenon causes measurement error as shown in fig. 22. This error may depend on both the optical configuration of the fibers and lens and surface shape of the lens, and cannot be eliminated by post numerical procedure. Consequently, optics should not be inserted in the optical path before the place where interference pattern is formed. Hence, the interference pattern is generated without optics as shown in fig. 23 and transferred to a CCD surface through a relay lens. In this configuration, the relay lens makes a copy of the interferogram on the CCD without adding aberrations to the fringe pattern on the CCD. In general, a rotating ground glass plate is used as the projection plane (Otaki et al., 2002a; Otaki et al., $2002 b$ ), but it may generate mechanical vibration. Instead of the ground glass, we tested a Fiber Optic Window (FOW) as the projection plane (Matsuura et al., 2007). The external view and magnified surface image of the FOW are shown in fig. 24 . The FOW is a bundle of multi-mode optical fibers, which transfers an optical image from the front side to rear side. This plate has several advantages: it transfers optical intensity distribution correctly to the rear side, the transferred image loses its coherency, and unwanted interference pattern does not occur on the CCD surface. In fig. 25, an experimental interferogram taken by the setup shown in fig. 23 is displayed. The interference pattern is vertically striped because the two fiber ends must be separated physically. Seven consecutive interference patterns are recorded in a $\Pi / 2$ phase-shift procedure, and the distortion of the test wavefront from the reference wavefront is calculated and shown in fig. 26. This figure should be zero for the entire area without measurement error because the test surface is the same as the reference surface. However, it exhibits long-range or low-frequency figure error across the entire area, meaning the error originated in the FOW. As shown in fig. 24, the fiber cladding of the FOW is thin, and crosstalk between the fibers is not negligible. Hence, the interference pattern is slightly deformed. This error is highly reproducible as exhibited in fig. 27, and subtraction of two results appears to eliminate the error perfectly. The difference between two datasets is shown in fig. 28. The FOW-induced long-range error is reduced adequately, and the vertically striped error pattern having twice the frequency of the interferogram is newly shown clearly. This may be a typical error usually caused by mechanical vibration in the phase-shift measurement (de Groot, 1995c; Creath \& Hariharan, 1994). By using seven successive interferograms with phase-shift errors, the same pattern is reproduced as shown in fig. 29. This may be due to the phase-shift error enhanced at the borders between bright and dark fringes where the intensity change is great. Mechanical vibration is a random variation, and the error shown in fig. 29 can be reduced by averaging many datasets. After eliminating the FOW-induced error and averaging 50 consecutive datasets, the difference between the two averaged datasets is shown in fig. 30 . The vibrational error shown in fig. 29 disappeared. A small residual error in fig. 30 distributes with the same period as the interferogram, which may be caused by changes in intensity of the interferogram during the phase-shift algorithm (van Wingerden et al., 1991). Fig. 30 is also reproduced by seven interferograms with slightly different intensities. The result is displayed in fig. 31. This error 
may be caused by relatively large and small intensity changes in bright and dark regions. Intensity fluctuation of the interferogram or laser light is caused by non-rigid components in the optical system, such as the laser-fiber coupler and fiber support. These effects are minimized by a long setup time before measurement. Finally, the difference between the two measurement datasets is shown in fig. 32, which is also the measurement accuracy of PS/PDI with the FOW. The remaining errors may be caused by residual errors of both vibration and intensity fluctuation and random noise in each pixel of the CCD detector. The FOW-induced error depends on the incident angle of light and is non-constant, and eliminating the error is difficult.

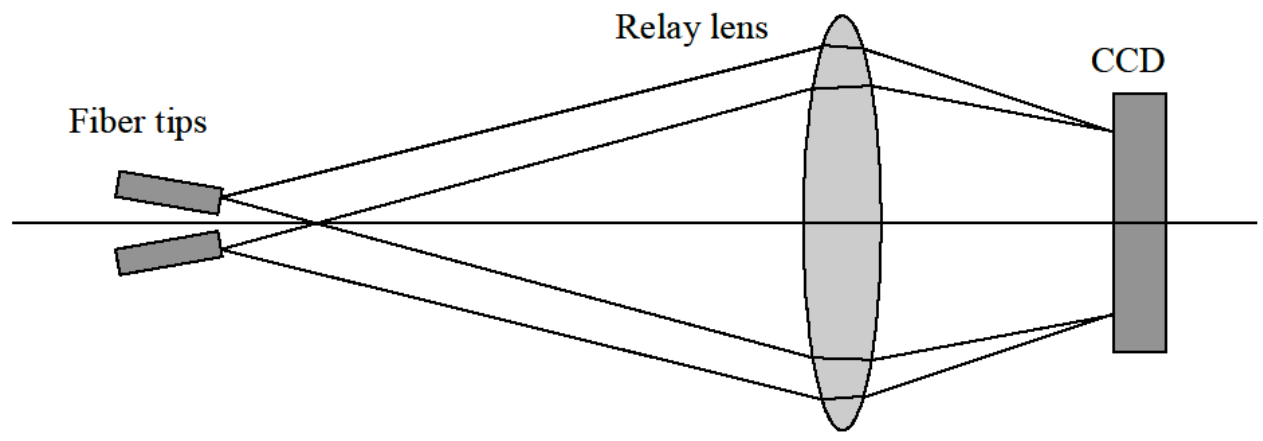

Fig. 20. Recording of interference pattern by two fiber ends with imaging lens.



Fig. 21. Change in optical path with and without flat-convex lens. 




Fig. 22. Error caused by aberration of flat convex lens.

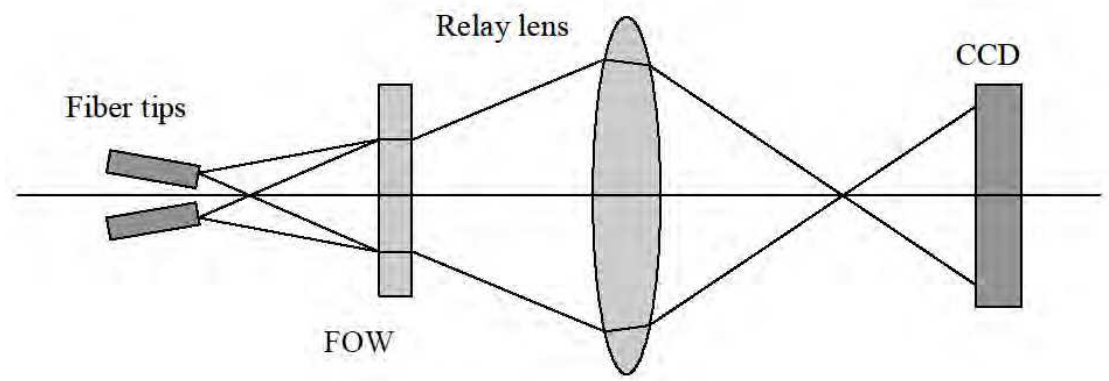

Fig. 23. Recording of interference pattern by FOW.
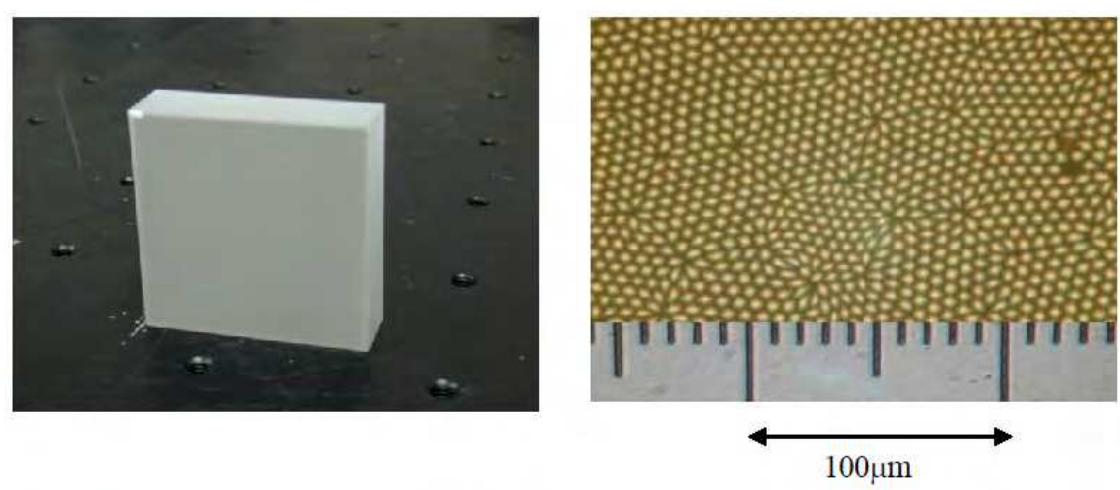

Fig. 24. Photo of the FOW plate and its microscopic surface image. 


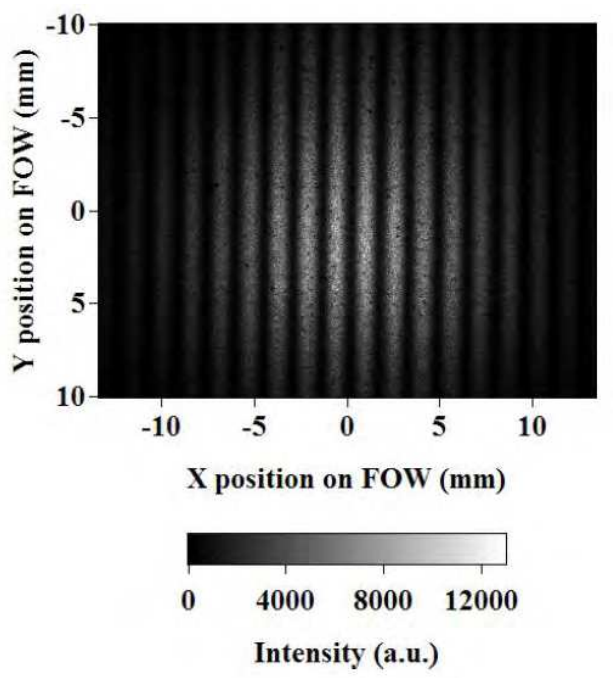

Fig. 25. Typical interference pattern recorded with the FOW plate.


Deviation (nm)

Fig. 26. Deviation of test wavefront from ideal sphere. 

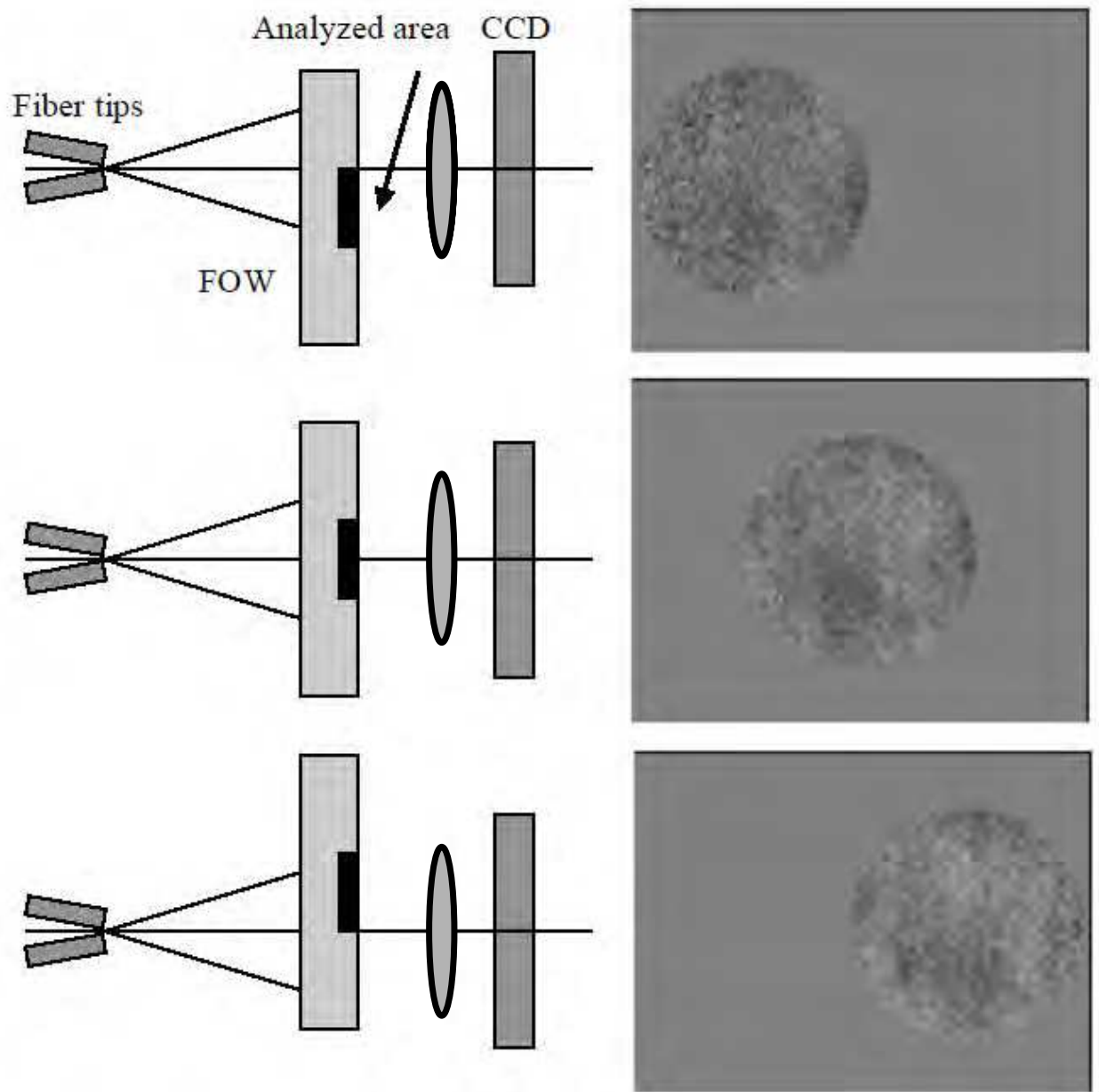

Fig. 27. Low frequency error caused by the FOW depends on its surface position. 


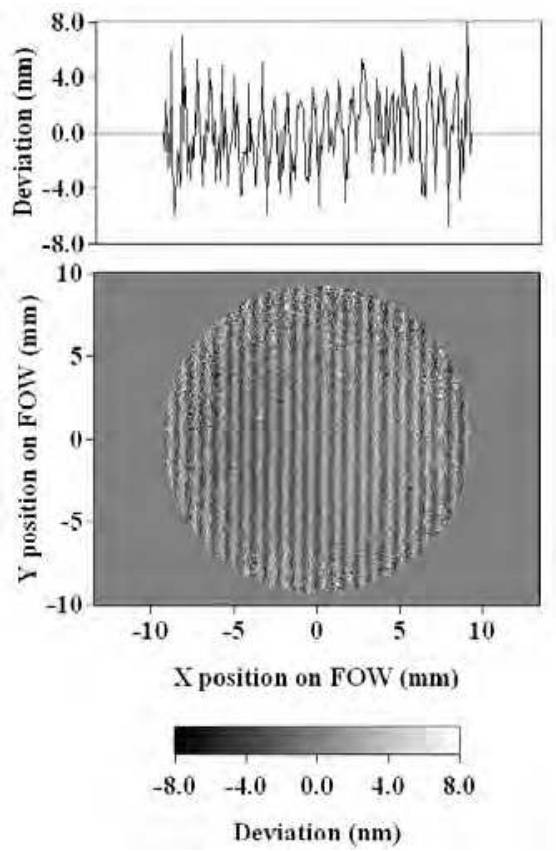

Fig. 28. Difference between two datasets taken with the FOW.

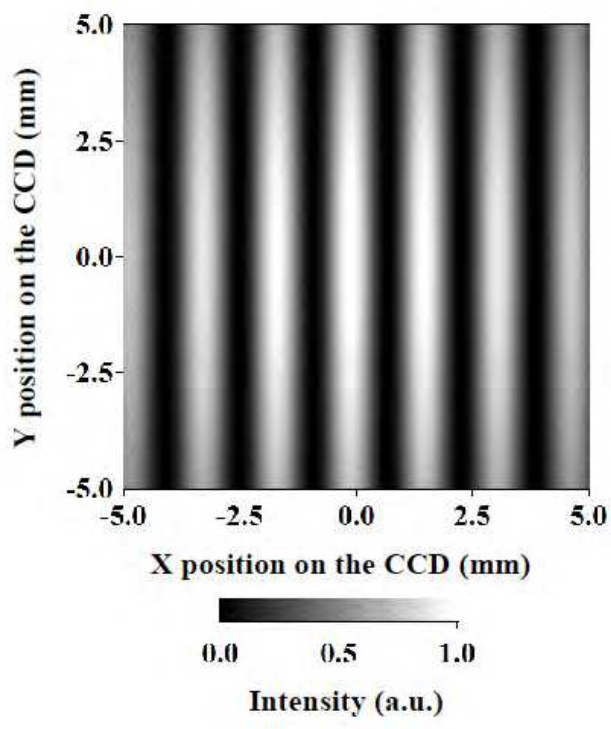

(a)

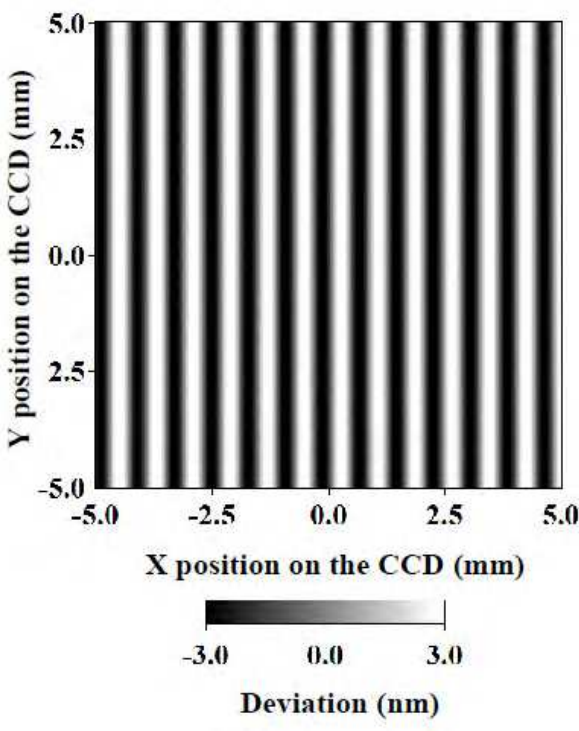

(b)

Fig. 29. Estimated phase error (a) in the PS analysis caused by error in movement of PZT stage with ideal interference pattern $(b)$. 

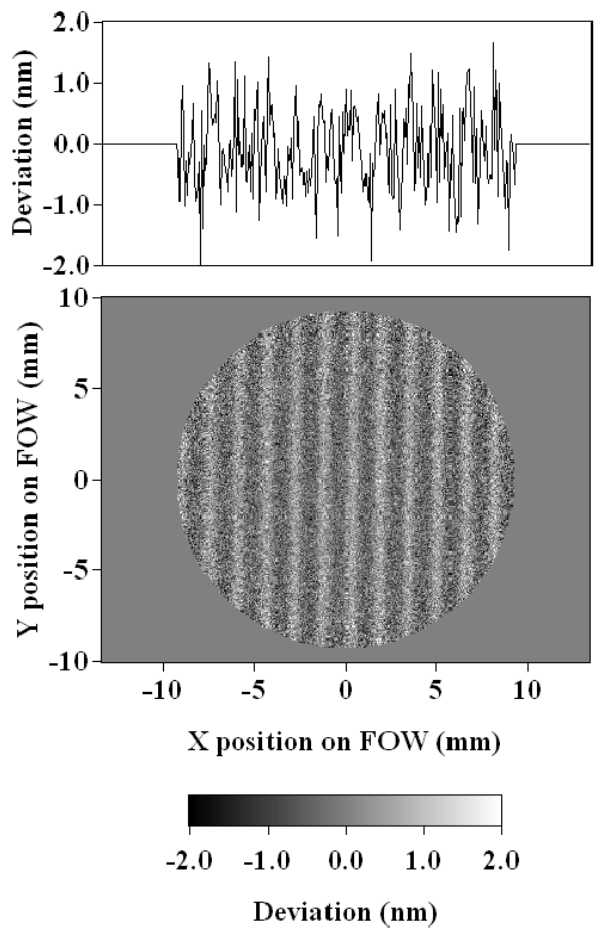

Fig. 30. Difference between two averaged datasets, which are averaged from 50 consecutive datasets.
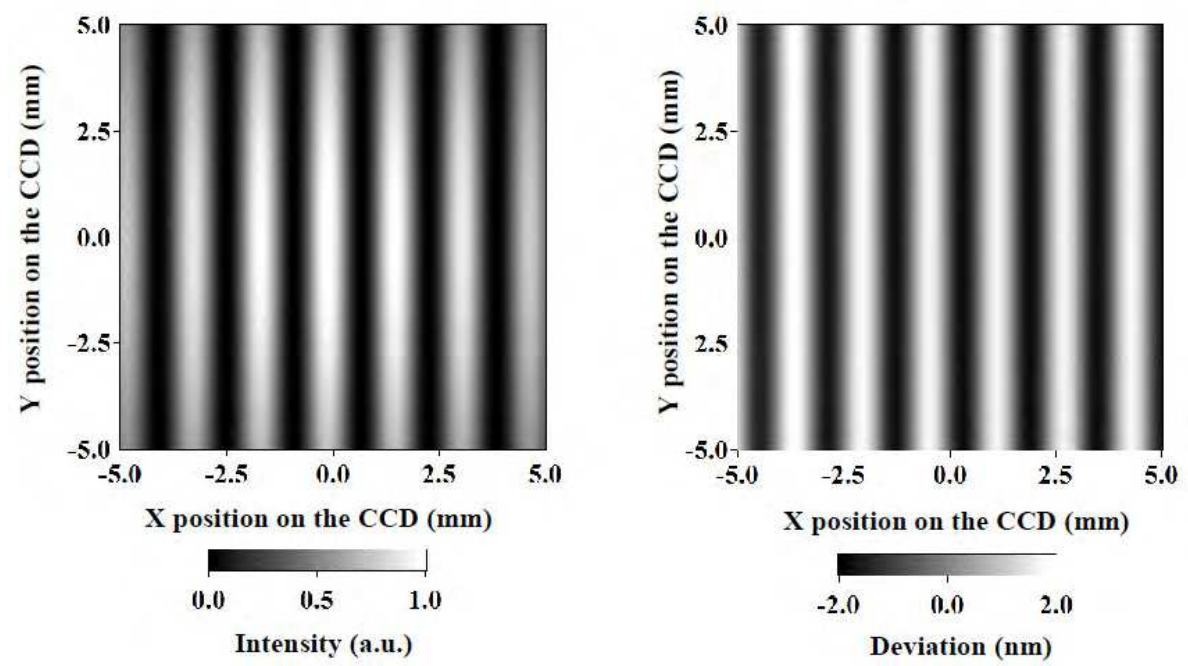

Fig. 31. Estimated phase error (right) in the PS analysis caused by fluctuation in laser light intensity. 

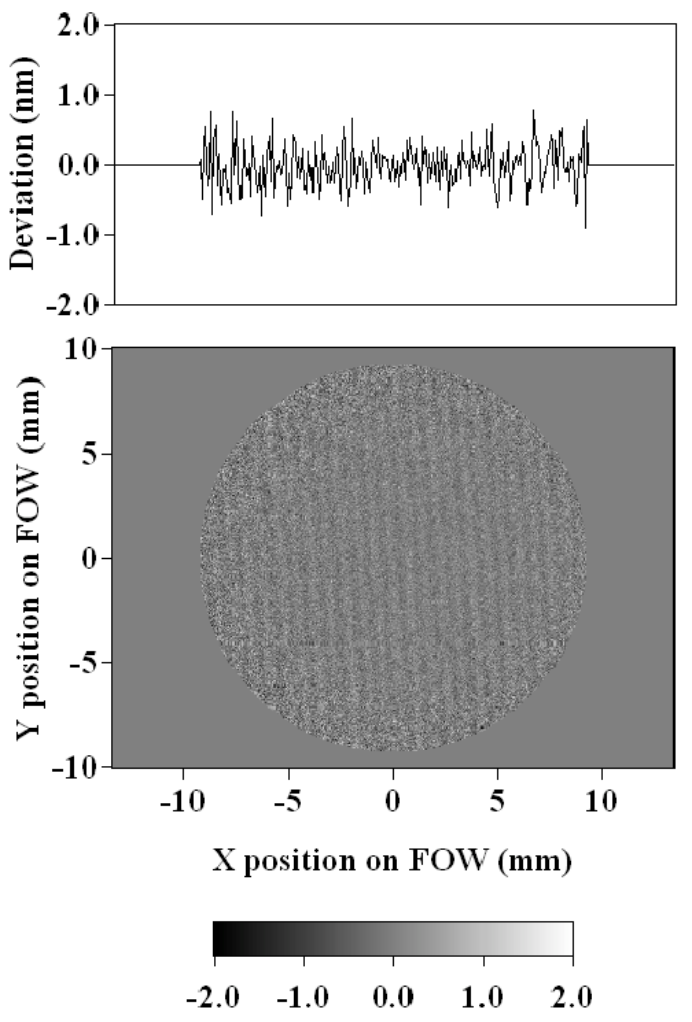

\section{Deviation (nm)}

Fig. 32. Difference between two averaged datasets with stabilized laser light, which are averaged from 50 consecutive datasets.

\subsection{Recording of interference pattern by glassless CCD camera}

In the previous section, measurement error caused by FOW is highlighted. Therefore, instead of the FOW, we constructed an alternative optical system without a lens and projection plane as shown in fig. 33. In the new configuration, the interference pattern is directly recorded by a CCD camera. In principle, this configuration requires system stability and random error control by averaging many datasets. In general, a CCD detector is covered by a glass plate to maintain the vacuum and for protection. Such a glass plate may cause internal reflection and unwanted interference inside the plate. Therefore, we adopted a glassless CCD camera without a protective glass plate. This CCD camera has considerable noise because it does not have a cooling system. To constrain the thermal and readout noises, each interference pattern processed by seven bucket algorithms is constructed by averaging 32 interference patterns at the same phase. Movement of the PZT stage, which actualizes phase shift by changing the optical path length accurately, is optimized to minimize the mechanical vibration of the phase-shift mirror. Thus, the movement is more segmentalized. The resultant deviation of the test surface from reference surface is shown in fig. 34(a), and 
its reproducibility is shown in fig. 34(b). These exhibit the measurement accuracy of this system. In fig. 34(a) and (b), vibrational error still exists. Here, an additional consideration is the condition of the fiber end. When the fiber end is polished poorly, fig. 34(a) becomes fig. 35. To confirm the sphericity of a diffraction wave, after the null test described above, one fiber end is rotated about its optical axis before a second null test. If both null tests return good results, the diffraction waves used in the PS/PDI are highly spherical. The test wave reflected off the test mirror has double the deformation information of the surface figure. Thus, half the distortion of the test wavefront from the ideal sphere corresponds to the mirror surface figure. For this reason, exact measurement accuracy of the PS/PDI is half the value shown in fig. 34. The accuracy is $1.6 \mathrm{~nm}$ PV and $0.17 \mathrm{~nm} \mathrm{rms}$ and the reproducibility is $0.7 \mathrm{~nm}$ PV and $0.07 \mathrm{~nm}$ rms.



CCD

Fig. 33. Recording of interference pattern without any optics.
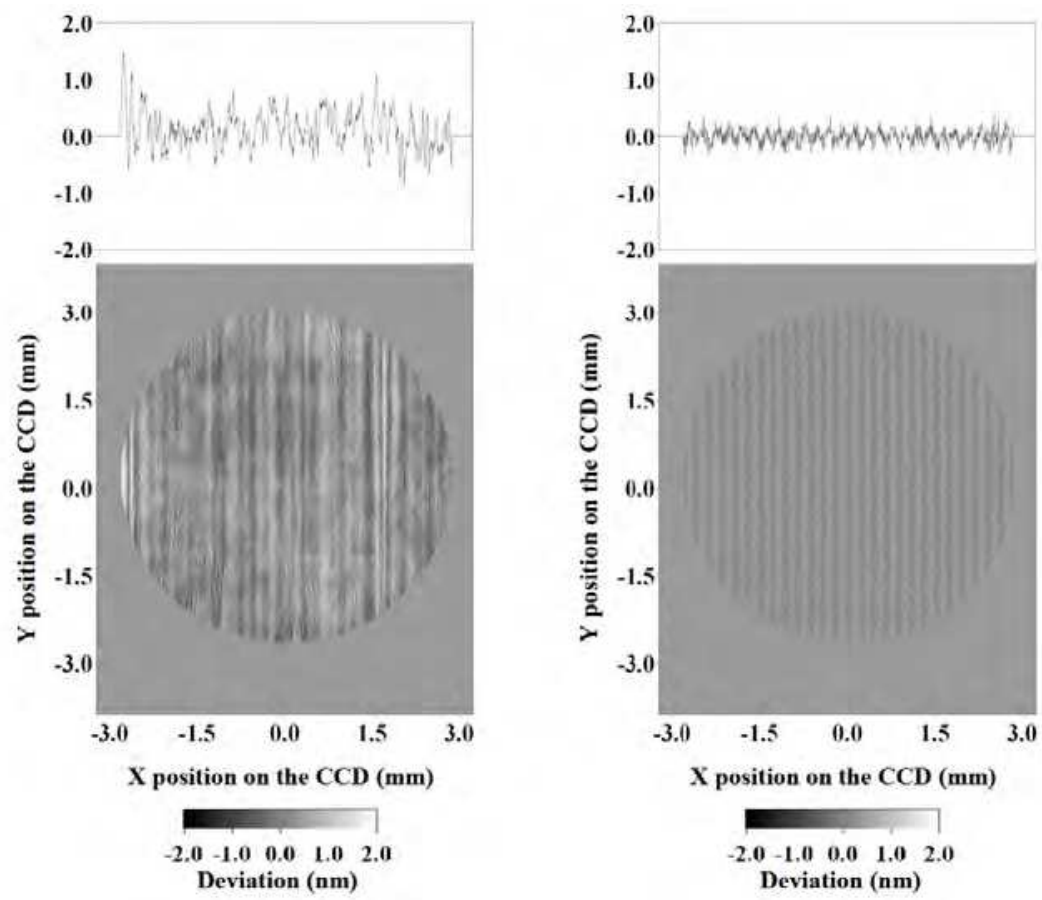

Fig. 34. Measurement accuracy of our PS/PDI system evaluated experimentally. Deviation of wavefront from ideal sphere (left) and the measurement reproducibility (right). 


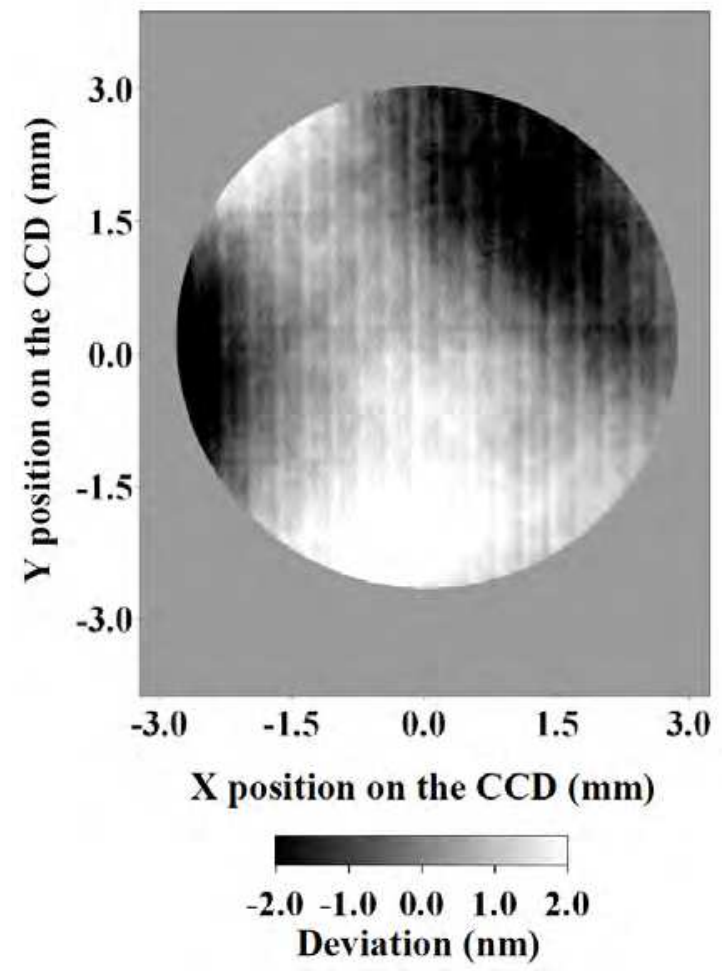

Fig. 35. Deviation of wavefront from ideal sphere caused by inadequate polish at fiber apex.

\section{Wavefront reconstruction on test mirror surface}

\subsection{Kirchhoff-Helmholtz integral theorem}

In the PS/PDI measurement or ordinary optical interferometry, the measurement result is a phase map of a wavefront including surface figure information of a test mirror on a CCD sensor. Here, reflection of light wave on a test mirror surface is considered in geometric optics. As shown in fig. 36, the propagation direction of each part of the reflected wave depends on angle of slope at the test mirror surface. In other words, the surface figure of the reflected wavefront depends on the observation or recording position, and the PS/PDI result with a glassless CCD camera will not agree with the exact surface figure of the test mirror. To obtain the actual mirror surface data, the reflected wavefront should be reconstructed on the test mirror surface using a digital holography technique with both phase and intensity maps computed from the CCD data (Yamaguchi et al., 2006; Matsuura et al., 2008). Fig. 37 shows a schematic diagram of the PS/PDI optics with two optical fibers. Planes $\mathrm{P}$ and $\mathrm{Q}$ are a spherical concave mirror and a projection plane for the interferogram, respectively. The test wave emitted from one fiber at point $\mathrm{O}$ propagates to test mirror $\mathrm{P}$, and the reflected wave converges on point $O$ and emanates to screen $Q$. The reference wave from another fiber end at point $O$ illuminates screen $Q$, and it interferes with the light wave from plane $\mathrm{P}$. The resultant interference pattern provides the surface figure of plane $\mathrm{P}$. If 
surface $P$ is highly spherical, the interference pattern on screen $Q$ will display the deviation of surface $P$ from an ideal sphere. In a practical sense, as explained in section 5.1, precise analysis is necessary for wavefront propagation. Here, a brief description of wave-optics analysis based on the Kirchhoff-Helmholtz $(\mathrm{KH})$ Integral Theorem follows. From the $\mathrm{KH}$ theorem, as shown in fig. 38, an optical wave field $U(Q)$, scalar value of electric or magnetic field, at point $\mathrm{Q}$ in closed surface $\mathrm{S}$ can be expressed with wave field $\mathrm{U}$ on closed surface $\mathrm{S}$ as Eq.(11) (Born \& Wolf, 1959).

$$
U(\mathrm{Q})=\frac{1}{4 \pi} \iint_{\mathrm{S}}\left[U \frac{\partial}{\partial \mathrm{n}}\left\{\frac{\exp (-\mathrm{i} k r)}{r}\right\}-\frac{\exp (-\mathrm{i} k r)}{r} \frac{\partial U}{\partial \mathrm{n}}\right] \mathrm{d} s
$$

Here, $\boldsymbol{r}$ is a vector between point $\mathrm{Q}$ and an arbitrary point on $\mathrm{S}$, and $\boldsymbol{n}$ is a unit normal vector at that point on S. In Eq.(11), the wave field on surface $\mathrm{P}$ is a reflected spherical wave emitted from point $\mathrm{O}$, and it is expressed as follows with a position vector $\boldsymbol{r}_{0}$.

$$
U=A \frac{\exp \left(i k r_{0}\right)}{r_{0}}
$$

Here, A is amplitude of wave field, and $k$ is wavenumber. Then, the directional derivative of Eq.(12) to the normal direction is as follows.

$$
\begin{aligned}
\frac{\partial U}{\partial n} & =\frac{\partial U}{\partial r_{0}} \frac{\partial r_{0}}{\partial n} \\
& =-A \frac{i k r_{0} \exp \left(i k r_{0}\right)-\exp \left(i k r_{0}\right)}{r_{0}^{2}} \quad\left(\because \frac{\partial r_{0}}{\partial n}=-1\right) \\
& =-A \exp \left(i k r_{0}\right) \frac{i k r_{0}-1}{r_{0}^{2}} \\
& =-A \frac{i k}{r_{0}} \exp \left(i k r_{0}\right) \quad\left(\because k r_{0} \gg>1\right)
\end{aligned}
$$

The following expression is also true.

$$
\begin{aligned}
& \frac{\partial}{\partial n}\left\{\frac{\exp (-i k r)}{r}\right\} \\
& \quad=\frac{\partial}{\partial r}\left\{\frac{\exp (-i k r)}{r}\right\} \frac{\partial r}{\partial n} \\
& =\frac{-i k r \exp (-i k r)-\exp (-i k r)}{r^{2}} \cos (\mathbf{n}, \mathbf{r}) \quad\left(\because \frac{\partial r}{\partial n}=\cos (\mathbf{n}, \mathbf{r})\right) \\
& =-\frac{i k r+1}{r^{2}} \exp (-i k r) \cos (\mathbf{n}, \mathbf{r}) \\
& \quad=-\frac{i k}{r} \exp (-i k r) \cos (\mathbf{n}, \mathbf{r}) \quad(\because k r>>1)
\end{aligned}
$$


Finally, Eq.(11) is modified as follows.

$$
\begin{aligned}
U(\mathrm{Q})=\frac{1}{4 \pi} \iint_{\mathrm{S}}[ & -A \frac{\exp \left(\mathrm{i} k r_{0}\right)}{r_{0}} \frac{\mathrm{i} k}{r} \exp (-\mathrm{i} k r) \cos (\mathbf{n}, \boldsymbol{r}) \\
& \left.+\frac{\exp (-\mathrm{i} k r)}{r} A \frac{\mathrm{i} k}{r_{0}} \exp \left(\mathrm{i} k r_{0}\right)\right] \mathrm{d} s \\
= & \frac{\mathrm{i} k}{4 \pi} \iint_{\mathrm{S}} A \frac{\exp \left(\mathrm{i} k r_{0}\right)}{r_{0}} \frac{\exp (-\mathrm{i} k r)}{r}\{1-\cos (\mathbf{n}, \boldsymbol{r})\} \mathrm{d} s
\end{aligned}
$$

These are wave analyses in the system of fig. 37. If the optical intensity of a spherical wave is uniform with no phase distortion, the term $A \exp \left(i k r_{0}\right) / r_{0}$ in Eq.(15) is constant. However, in an actual case, the test surface is aspherical due to a machining error, and intensity distribution of the diffraction wave is non-uniform. These two matters must be considered in the reconstruction procedure of the wavefront on the test mirror surface. Now, as shown in fig. 39, an orthogonal coordinate system is positioned with its origin at an arbitrary point on spherical $\mathrm{P}$, and the $\mathrm{x}-\mathrm{y}$ plane is a tangential plane of spherical P. Hence, in fig. 39, the normal vector $\boldsymbol{n}$ faces the negative direction of the z-axis. Using this coordinate system, the surface figure of the wavefront near the origin is expressed as follows.

$$
z=z_{0}(x, y)
$$

Propagation direction of the wavefront may be its normal direction, and a normal unit vector $\mathbf{n}_{\mathrm{w}}$ is introduced at the intersection point between the $\mathrm{z}$-axis and wavefront. Instead of Eq.(12), wave field of the reflected wave is as follows.

$$
U=A \exp \left\{i k z_{0}(x, y)\right\}
$$

And the related expressions are derived as follows.

$$
\begin{gathered}
\frac{\partial U}{\partial n}=\frac{\partial U}{\partial z_{0}} \frac{\partial z_{0}}{\partial n} \\
=A i k \exp \left(i k z_{0}\right) \cos \left(\mathbf{n}, \mathbf{n}_{W}\right) \\
U(\mathrm{Q})=\frac{1}{4 \pi} \iint_{\mathrm{s}}\left[-A \exp \left(\mathrm{i} k z_{0}\right) \frac{\mathrm{i} k}{r} \exp (-\mathrm{i} k r) \cos (\mathbf{n}, \boldsymbol{r})\right. \\
\left.-\frac{\exp (-\mathrm{i} k r)}{r} A \mathrm{i} k \exp \left(\mathrm{i} k z_{0}\right) \cos \left(\mathbf{n}, \mathbf{n}_{\mathrm{w}}\right)\right] \mathrm{d} s \\
=-\frac{\mathrm{i} k}{4 \pi} \iint_{\mathrm{S}} A \exp \left(\mathrm{i} k z_{0}\right) \frac{\exp (-\mathrm{i} k r)}{r}\left\{\cos \left(\mathbf{n}, \mathbf{n}_{\mathrm{w}}\right)+\cos (\mathbf{n}, \boldsymbol{r})\right\} \mathrm{d} s
\end{gathered}
$$


Using these equations, if the wave field on surface $P$ is obtained as described in Eq.(17), the wave field on surface $Q$ can be calculated by integration. Finally we develop the expression for the terms $\cos (\mathbf{n}, \mathbf{r})$ and $\cos \left(\mathbf{n}, \mathbf{n}_{\mathrm{W}}\right)$. Referring to fig. $37, \cos (\mathbf{n}, \mathbf{r}), \mathbf{n}$, and $\mathbf{e}_{\mathrm{r}}$ are expressed as follows.

$$
\begin{gathered}
\cos (\mathbf{n}, \boldsymbol{r})=\mathbf{n} \cdot \mathbf{e}_{r} \\
\mathbf{n}=\frac{\left(-x_{\mathrm{p}},-y_{\mathrm{p}},-z_{\mathrm{p}}\right)}{\sqrt{x_{\mathrm{p}}{ }^{2}+y_{\mathrm{p}}{ }^{2}+z_{\mathrm{p}}{ }^{2}}} \\
\mathbf{e}_{r}=\frac{\left(x_{\mathrm{p}}-x_{\mathrm{q}}, y_{\mathrm{p}}-y_{\mathrm{q}}, z_{\mathrm{p}}-z_{\mathrm{q}}\right)}{\sqrt{\left(x_{\mathrm{p}}-x_{\mathrm{q}}\right)^{2}+\left(y_{\mathrm{p}}-y_{\mathrm{q}}\right)^{2}+\left(z_{\mathrm{p}}-z_{\mathrm{q}}\right)^{2}}} \\
\cos (\mathbf{n}, \boldsymbol{r})=-\frac{x_{\mathrm{p}}\left(x_{\mathrm{p}}-x_{\mathrm{q}}\right)+y_{\mathrm{p}}\left(y_{\mathrm{p}}-y_{\mathrm{q}}\right)+z_{\mathrm{p}}\left(z_{\mathrm{p}}-z_{\mathrm{q}}\right)}{\sqrt{\left(x_{\mathrm{p}}{ }^{2}+y_{\mathrm{p}}{ }^{2}+z_{\mathrm{p}}{ }^{2}\right)^{2}\left(x_{\mathrm{p}}-x_{\mathrm{q}}\right)^{2}+\left(y_{\mathrm{p}}-y_{\mathrm{q}}\right)^{2}+\left(z_{\mathrm{p}}-z_{\mathrm{q}}\right)^{2}}}
\end{gathered}
$$

And referring to fig. $38, \cos \left(\mathbf{n}, \mathbf{n}_{\mathrm{W}}\right), \mathbf{e}_{\mathrm{z}}$, and $\mathbf{n}_{\mathrm{W}}$ are expressed as follows.

$$
\begin{gathered}
\cos \left(\mathbf{n}, \mathbf{n}_{w}\right)=-\mathbf{e}_{z} \cdot \mathbf{n}_{\mathrm{w}} \\
\mathbf{e}_{z}=(0,0,1) \\
\mathbf{n}_{\mathrm{w}}=\frac{\left(-\frac{\partial z_{0}}{\partial x},-\frac{\partial z_{0}}{\partial y}, 1\right)}{\sqrt{1+\left(\frac{\partial z_{0}}{\partial x}\right)^{2}+\left(\frac{\partial z_{0}}{\partial y}\right)^{2}}}
\end{gathered}
$$

Finally, Eq.(27) is obtained.

$$
\cos \left(\mathbf{n}, \mathbf{n}_{w}\right)=-\frac{1}{\sqrt{1+\left(\frac{\partial z_{0}}{\partial x}\right)^{2}+\left(\frac{\partial z_{0}}{\partial y}\right)^{2}}}
$$

Here, after reflection at surface $\mathrm{P}$, the reflected wave propagates in the negative direction of the z-axis because of Eq.(17).

\subsection{Wave propagation in measurement of perfect spherical surface}

Changes in wavefront via propagation are simulated numerically based on the $\mathrm{KH}$ theorem as described in the previous section. When the simulation condition is set, light intensity and phase maps of the test wave on the test mirror are determined. As the simulation is conducted, the test wavefront on the CCD sensor is calculated using the $\mathrm{KH}$ theorem, and the resultant phase map as an ordinary PS/PDI result is compared with the reconstructed phase map on the test mirror surface. In the calculation, discretization of the integral is 


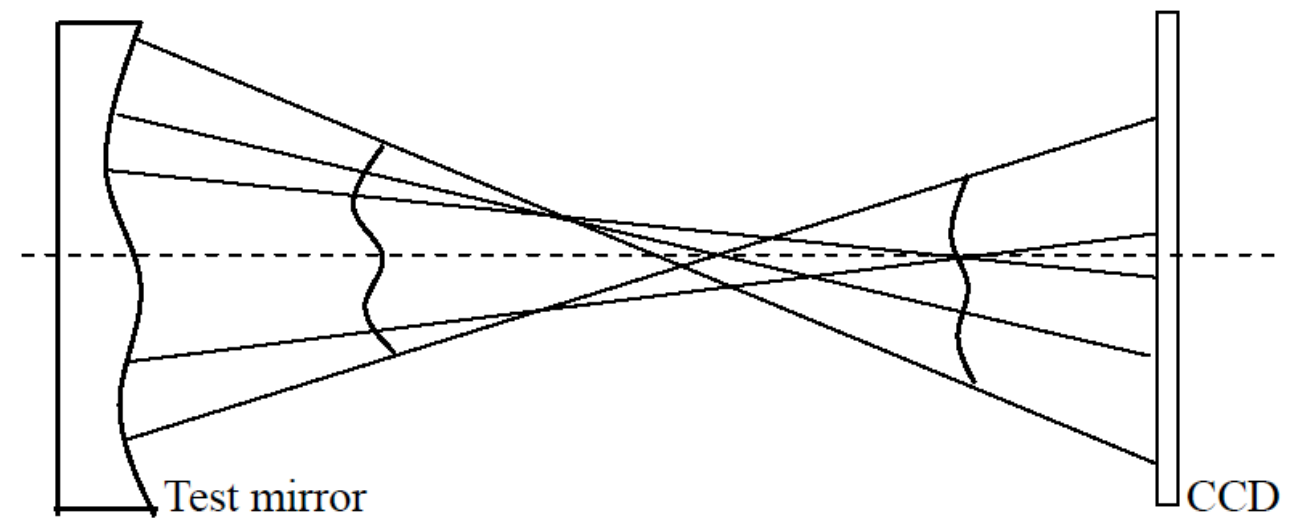

Fig. 36. Correspondence relation between points on test mirror and CCD surface.

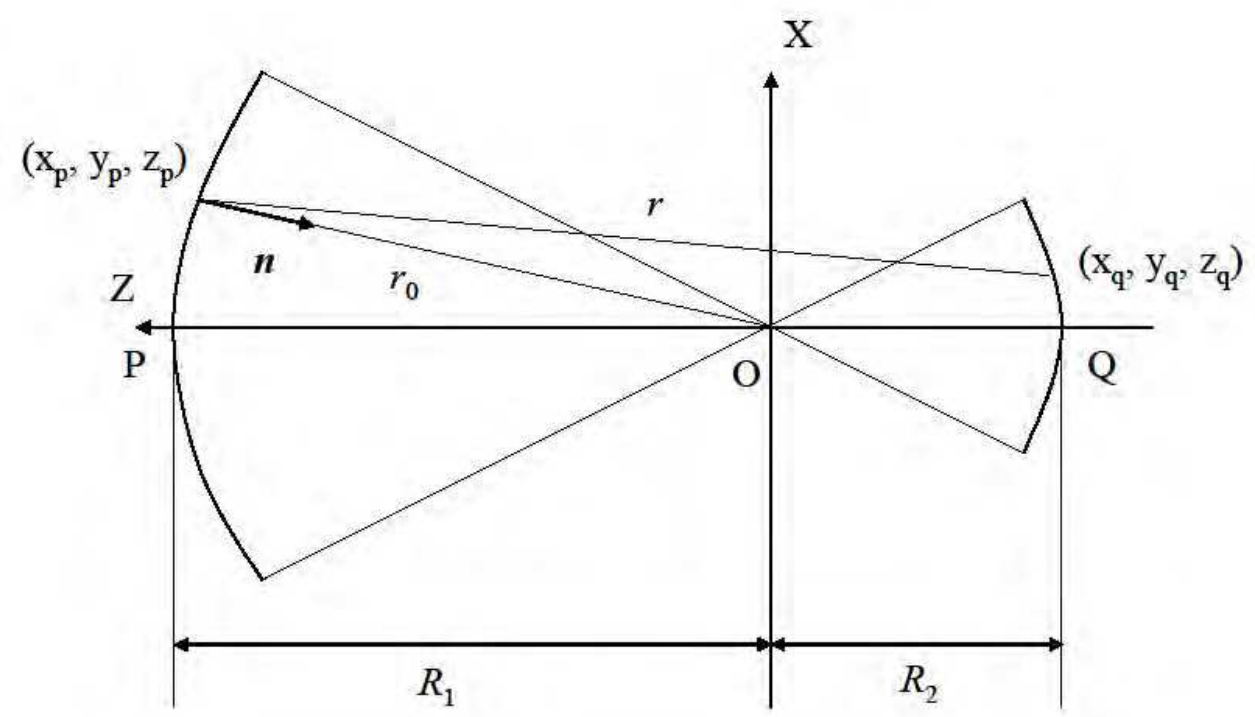

Fig. 37. Mathematical model for the PDI system. 




Fig. 38. Domain of integration in $\mathrm{KH}$ integral theorem.

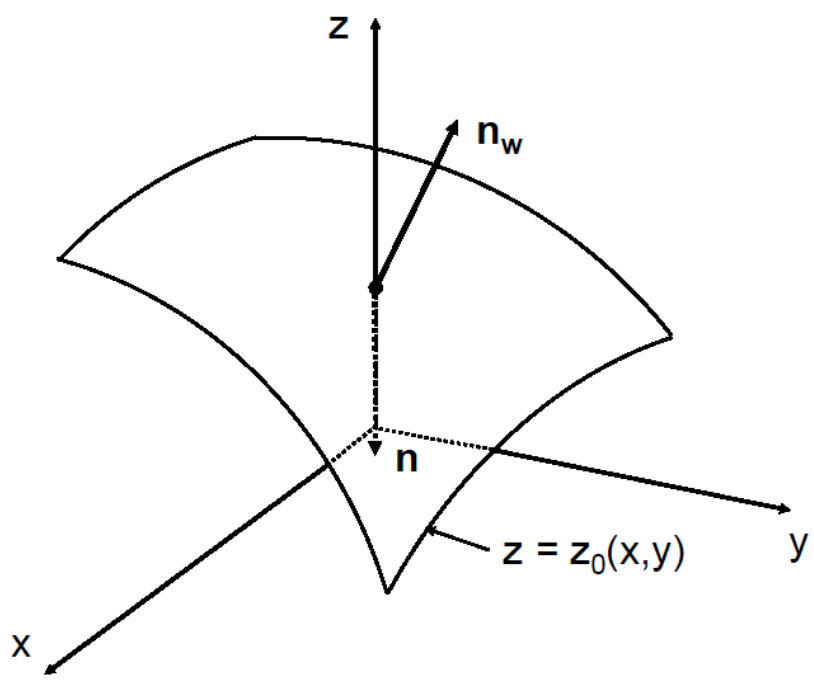

Fig. 39. Wavefront around a point on sphere P.

required, and a sufficient number of discretized integral terms are needed for accurate solution. The actual mirror surface is divided into $5000 \times 5000$ pixels as the integral terms. In contrast, the CCD surface is divided into $400 \times 400$ pixels for convenience of calculation time. The reconstruction process for a wavefront at the test mirror requires a sufficient amount of data on the CCD surface, and the $400 \times 400$ dataset is interpolated. The resultant finer data of $5200 \times 5200$ pixels are used to reconstruct the wavefront on the test mirror surface in $400 \times$ 400 pixels. The simulation described above is completed with an ideal spherical concave mirror based on the configuration shown in fig. 40. The test mirror is $180 \mathrm{~mm}$ in diameter and has a 1500-mm ROC. The origin of the diffraction wave is positioned at the center of curvature of the mirror. The wavelength of the light wave is $632.8 \mathrm{~nm}$. The CCD surface is 
assumed to be spherical concave with a $150 \mathrm{~mm} \mathrm{ROC}$, and it is concentric with the mirror surface. Light intensity distribution on the diffraction wavefront is determined as the Gaussian distribution shown in fig. 41(a). With the uniform phase map on the mirror surface and fig. 41(a), wavefront on the CCD surface is simulated as shown in fig. 41(b). It corresponds to the ordinary PS/PDI result, and is vaulted entirely. The phase map near the mirror edge fluctuates erratically, and an unexpected pattern appeared. Using the resultant intensity distribution and phase maps on the CCD surface, the wavefront was reconstructed on the test mirror surface. As a result, the mirror surface figure or phase map is calculated as shown in fig. 41(c), and the phase map is uniform in most of the area. Huge fluctuations in the phase map appeared near the edge. Next, the same simulation is performed with the more sharpened intensity distribution shown in fig. 42(a). Fig. 42(b) shows the result, which is also vaulted but the phase disturbance near the edge is reduced effectively. The reconstructed wavefront, as shown in fig. 42(c), has a uniform phase map in most area. However, huge fluctuations of the phase appeared near the edge region. In the above simulations, an integral corresponds to superposition of the weighted diffraction waves emitted from everywhere in the integral range. The weighting depends on intensity distribution. Hence, two different results were obtained and are shown in figs. 41 and 42. In fig. 42, contribution of the central part of the wavefront is effective due to its sharpened intensity distribution profile. Large fluctuations in phase maps may be caused by both diffraction at the mirror edge and insufficient discretization of the integral range. In the latter case, light intensity is weak at the mirror edge, and the edge effects are highly suppressed. The phase map in the edge area includes both systematic error from numerical calculations (Greivenkamp, 1987) and Moire fringes caused by grid-like segmentalization in the integral range (Ferraro et al., 2007). The same phenomenon appears in fig. 42. Reconstruction of the low frequency component results in a correct solution, while reconstruction of the high frequency component has considerable errors. In the latter case, the wavefront in only the central square region is correctly restored due to extremely low light intensity near the edge and the domain of integration is confined in the square region. In principle, the $\mathrm{KH}$ integral requires a closed surface for its integral domain. However, in these simulations, most of the reflected light propagates inside the square region, and an unhandled component of the wavefront is negligible. Therefore, the wavefront reconstruction processes are successfully achieved as shown in fig. 41 and 42 . However, in fig. 42, light intensity in the outer edges of the test mirror is extremely low, and the subsequent lack of wavefront information caused incorrect solution of outer edges in the reconstruction procedure.

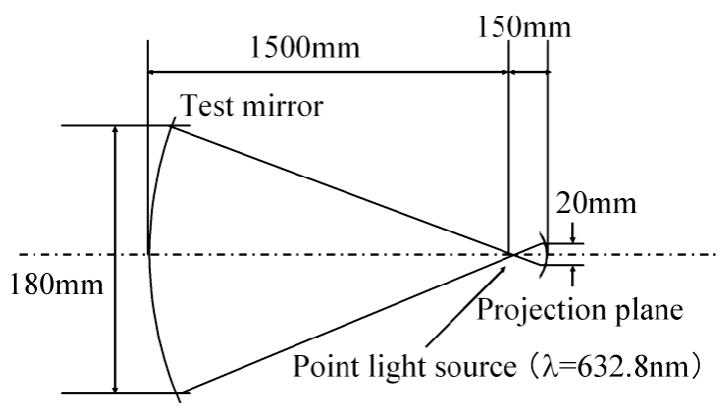

Fig. 40. Mathematical model of PS/PDI for numerical simulation. 


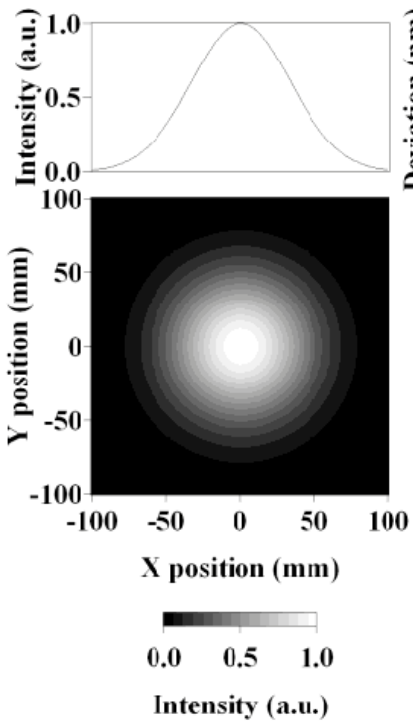

(a)



$\mathrm{X}$ position ( $\mathrm{mm})$



Deviation (nIm)

(b)

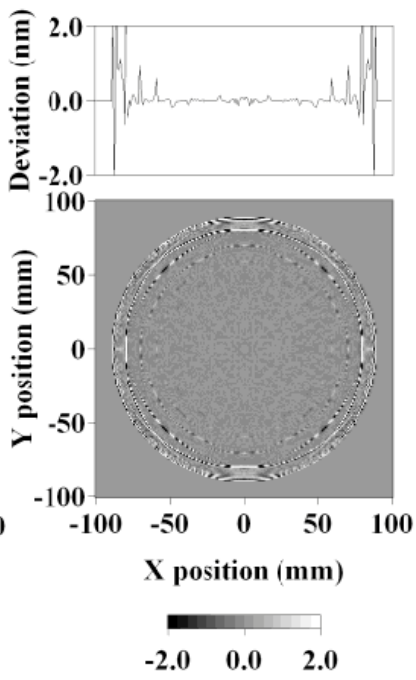

Deviation (nm)

(c)

Fig. 41. Wave propagation simulation with ideal spherical concave mirror. (a) light intensity distribution, (b) phase map on CCD, and (c) reconstructed phase map on mirror surface.
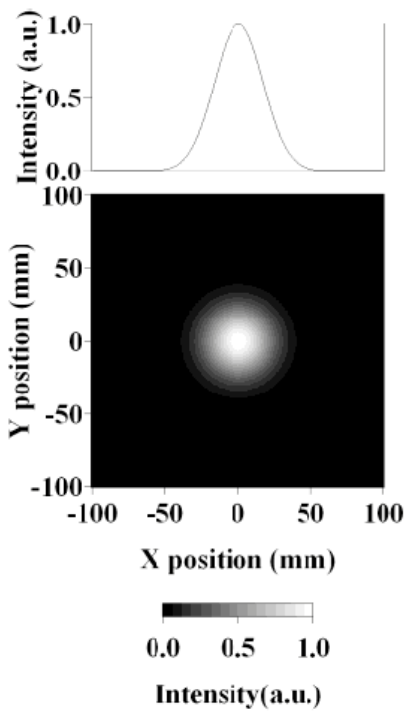

(a)



$\begin{array}{rlllll}-5 & 0 & 5 & 10 & 15\end{array}$

Deviation (nm)

(b)

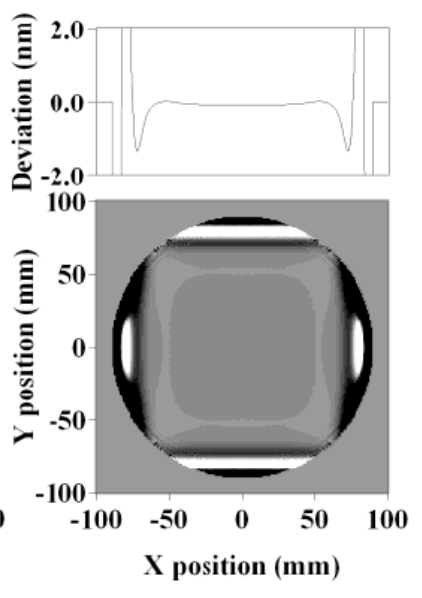

$-2.0 \quad 0.0 \quad 2.0$

Deviation (nm)

(c)

Fig. 42. Wave propagation simulation with ideal spherical concave mirror. (a) sharpen intensity distribution of light, (b) phase map on CCD, and (c) reconstructed phase map on mirror surface. 


\subsection{Wave propagation in measurement of imperfect spherical surface}

An actual test mirror may have errors at different spatial frequencies. In this section, wave propagation analysis for test mirrors having surface figure error at both high and low spatial frequencies is conducted, and reconstruction of the wavefront is completed. Here, the reflected wave emanates in diverse directions, and a distant projection plane or CCD surface might not be able to collect most of the reflected light. Therefore the CCD surface is located at a distance of $15 \mathrm{~mm}$ from the focal point of the reflected wave. The test mirror surface has a high frequency surface figure deviation from an ideal sphere, similar to a Gaussian profile in the central portion as shown in fig. 43(a) and (d). The maximum deviation is $83.3 \mathrm{~nm}$ from ideal surface as shown in fig 43(d), and this deviation profile corresponds approximately to a flat surface $1 \mathrm{~mm}$ in diameter. The intensity profile of the diffraction wave is the same as that of fig. 41(a), and the wave propagation analysis provides the test mirror surface figure data as a phase map on the CCD sensor shown in fig. 43(b). A part of the diffraction wave reflected at the central flat area on the test mirror surface continues spreading, and not a trace of the central small area is visible in fig. 43(b). However, the reconstruction process with these data results test the mirror figure as shown in fig. 43(c), and the central crosssection profile shown in fig. 43(e) exhibits approximately the same profile as fig. 43(d). The wavefront reconstruction process succeeded in reproducing the exact surface figure with high frequency error in the numerical simulation. Next, we assume a test surface with lowfrequency surface error of sinusoidal fluctuation of $50 \mathrm{~nm}$ in amplitude in the entire area as shown in fig. 44(a). With the same intensity distribution as fig. 41(a), the wavefront reconstruction process provides the solution indicated in fig. 44(b) and (c), which are phase maps on the CCD surface and the reconstructed wavefront, respectively. In both cases, lowfrequency surface figure error is reproduced successfully. Therefore, an exact surface figure having low frequency error can be obtained only from a phase map on the CCD, according to underlying basic optics. However, an actual mirror having different kinds of frequency components in its error profile must be measured by a PS/PDI system with a wavefront reconstruction procedure.



(a)



(b)

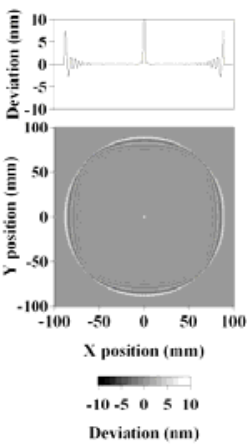

(c)

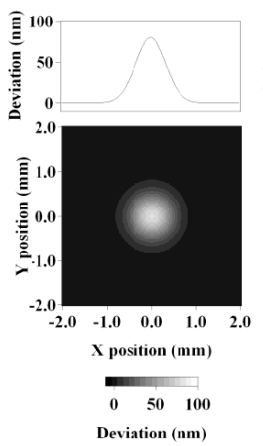

(d)

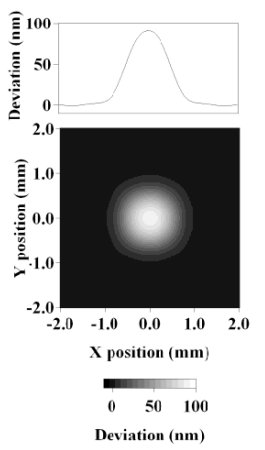

(e)

Fig. 43. Wavefront propagation analysis with test mirror surface having figure error in central area at high spatial frequency. (a) test mirror surface figure, (b) wavefront obtained on CCD surface, (c) wavefront reconstructed on test mirror surface, (d) magnified image of surface figure error in (a), and (e) magnified image of reconstructed surface figure error in the analysis. 




Deviation $(\mathbf{n m})$

(a)

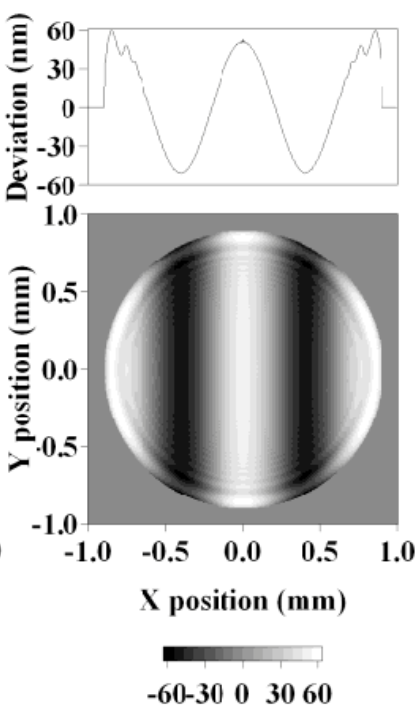

Deviation $(\mathrm{nm})$

(b)



Deviation (nm)

(c)

Fig. 44. Wavefront propagation analysis with test mirror surface having figure error in whole surface at low spatial frequency. (a) test mirror surface figure, (b) wavefront obtained on CCD surface, and (c) wavefront reconstructed on test mirror surface.

\section{Measurement of spherical concave mirror}

\subsection{Procedures for measurement and analysis}

The PS/PDI system is advantageous in the measurement of a spherical concave mirror because its reference plane is an ideal sphere for the diffraction wavefront. In this section, trial measurement of a conventional spherical concave mirror $200 \mathrm{~mm}$ in diameter, $1500 \mathrm{~mm}$ in ROC, and with $\lambda / 6$ figure accuracy is reported with reproducibility of the measurement with and without change in the mirror alignment (Matsuura et al., 2010). Firstly, the supporting technique is examined with an ordinary CCD camera and imaging lens. The test mirror described above is epoxied onto an aluminum block ( $300 \mathrm{~mm} \times 300 \mathrm{~mm} \times 80 \mathrm{~mm}$ ) and supported by three-point mounting with steel balls for highly rigid supporting. The block is supported by an aluminum thin plate mounted between its top face and supporting rod stands on an optical bench to control mechanical vibration. A photo of the mirror apparatus is shown in fig. 45 . On the outer edge of mirror surface, three triangular black papers serving as position markers are attached, and the measurement is completed inside the marks. In figs. 46, 47, and 48, both the interference pattern and phase map on the CCD are shown for three different ambient temperatures of the PS/PDI measurement. Temperature change caused stress at the supporting ball due to the difference in the coefficient of thermal expansion (CTE) between the mirror substrate and aluminum block. Hence, the three phase maps on the CCD are slightly altered. Their differences are indicated in figs. 49 and 50. A temperature change of $2.7^{\circ} \mathrm{C}$ caused surface deformation of $\pm 20 \mathrm{~nm}$ in 
the diffraction wavefront. The CTE for aluminum and Pyrex glass are $29 \times 10^{-6}\left[\mathrm{~K}^{-1}\right]$ and $3.0-$ $3.6 \times 10^{-6}\left[\mathrm{~K}^{-1}\right]$, respectively, and the difference is enough to cause considerable stress for such a deformation. Next, the test mirror is placed with its side surface resting on three steel balls, and the top edge is touched by the apex of a needle to control mechanical vibration. The mirror supported by this method is shown in fig. 51 . To examine the effect of the supporting needle, a series of 60 measurement datasets were taken and each ten datasets were averaged to form an average map, and the resultant six images are displayed in fig. 52. Moreover, each average map is numerically approximated to a plane, and the change in direction of the surface normal vector is plotted in fig. 53. In fig. 52, there is no remarkable change. However, tilt of the mirror surface is clearly changing, especially in the vertical direction, as exhibited in fig. 53. In fact, thermal expansion of the supporting needle causes tilt change of test mirror surface. As a result, the three-point steel ball support system causes not issues with the mirror measurement when it is combined with a thermal control system, such as a temperature-controlled room. Second, in actual PDI measurement, which differs from the null test in section 4, a common-path interferometry will not be realized because only the test wave propagates along the optical path through the measurement atmosphere, and the influence of air fluctuation is experimentally studied by collecting 100 consecutive datasets. Fig. 54 shows change in each averaged dataset, which is calculated from 10 consecutive datasets. A low frequency error pattern is moving from map-to-map, which may be caused by air current. The actual PS/PDI measurement is performed with the random error averaged out. In the following, a glassless CCD camera is used as mentioned in section 4, and the wavefront reconstruction process is employed according to the discussion in section 5. Each interference pattern at a different phase is composed of 32 averaged patterns for reduction of the CCD noise, and the step of phase shift is $\lambda / 2$. A frequently used seven bucket algorithm is used to determine an initial phase map, which is averaged over 100 consecutive datasets to eliminate random noise such as air current. With location data of the fibers and CCD determined by pre-experiment analysis, the test wavefront on the CCD surface is calculated. Finally, reconstruction of the wavefront determines the exact surface figure of the test mirror. In figs. 55 and 56, a typical light intensity distribution and an interference pattern are exhibited, respectively. In the images, the lower part is misshapen due to the tip-resistant cover over the mirror surface. Coaxial fringes at the outer edge of the image are caused by edge diffraction. The position markers produced three circular patterns in fig. 55. Tilt fringes or vertical stripes caused by a position gap between the reference fiber end and the focal spot of the test wave are dominant in fig. 56. Location data of the fibers and CCD are derived by the analytical method mention in section 4 since the interference pattern is nearly ideal. The wavefront is shown in fig. 57 as the pattern deviation from an ideal sphere. From 100 consecutive datasets, the difference between the averaged first 50 and second 50 datasets is presented in fig. 58, and they agree within $0.11 \mathrm{~nm}$ rms. In section 4 , measurement reproducibility was estimated to be $0.07 \mathrm{~nm} \mathrm{rms}$, and the lower value may be caused by air current in the optical system. Before the wavefront reconstruction procedure, location data based on the interference between two ideal spherical waves are evaluated by reconstructing the intensity profile at the focal point of the test wave. In fig. 59(a) and (b), the derived profile and an ideal profile are displayed, respectively. The data confirms that the reflected wavefront is focused well and is fairly spherical. In the final step, the exact location of the test mirror surface is required. The position coordinates are derived from the focal position of the test wave and the ROC of the mirror specification data, i.e. $1500 \mathrm{~mm}$. Fig. 60 shows the resultant reconstructed intensity profile and surface figure of the 
test mirror. The derived profile at $Y=0 \mathrm{~mm}$ in fig. 60 is compared with the actual intensity profile of a diffraction wave as shown in fig. 61 . Within a $170 \mathrm{~mm}$ radius of the mirror, the profiles agree well, and the wavefront reconstruction succeeded, while the outer edge data is not good due to a lack of wavefront data outside the CCD sensor.

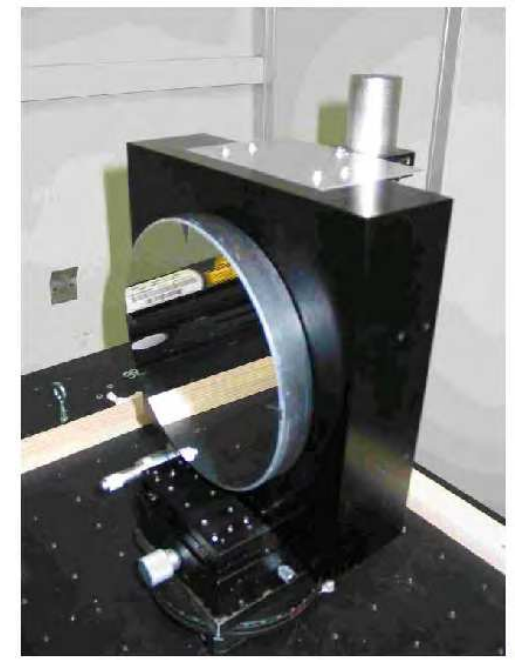

Fig. 45. Mirror supported by three-point bonding on aluminum block.
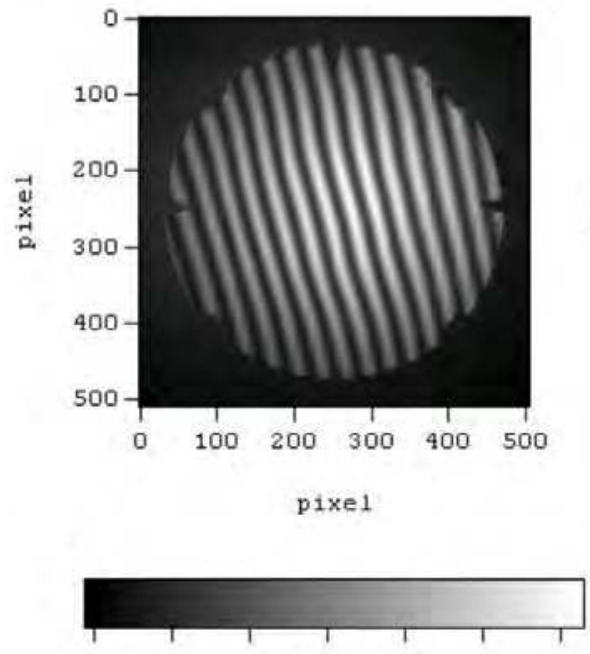

$\begin{array}{lllllll}0 & 125 & 250 & 375 & 500 & 625 & 750\end{array}$

Intensity (count)
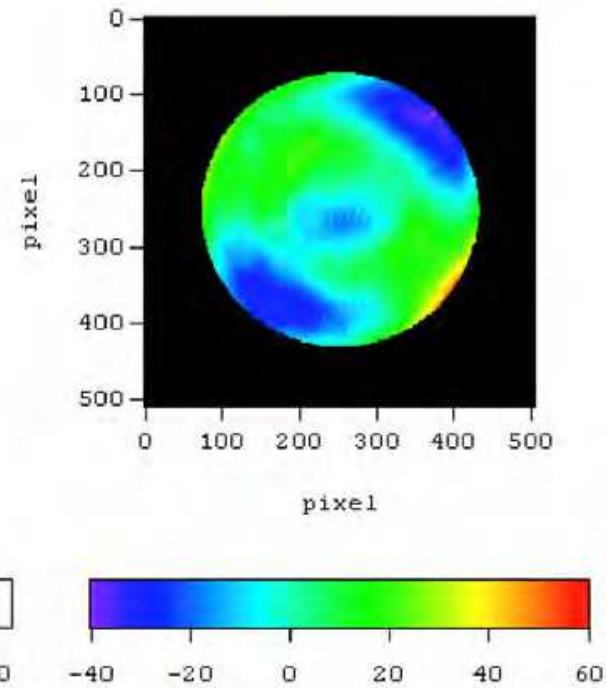

Deviation (nm)

Fig. 46. Interference pattern and measured phase map on CCD surface at ambient temperature of 23.46 degrees Celsius. 

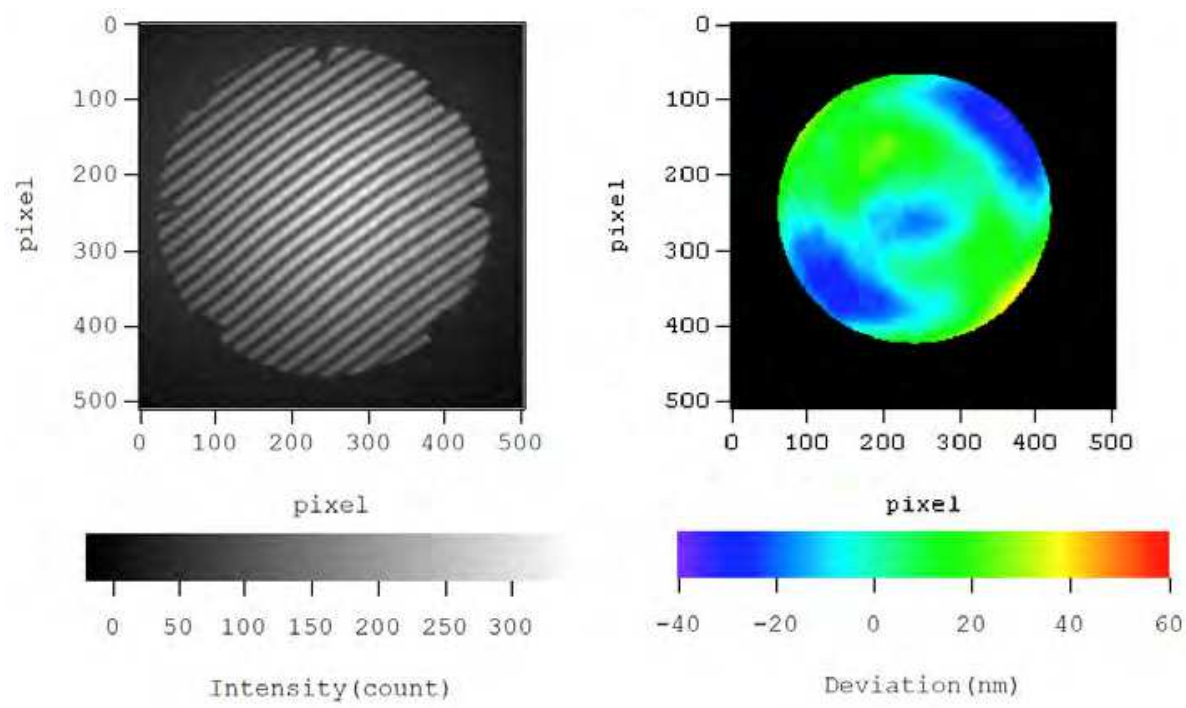

Fig. 47. Interference pattern and measured phase map on CCD surface at ambient temperature of 20.77 degrees Celsius.

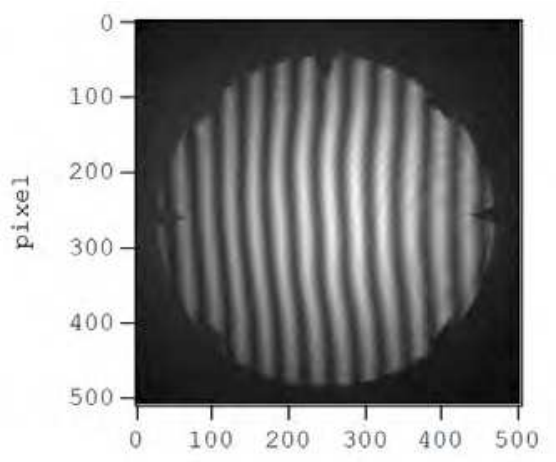

pixel

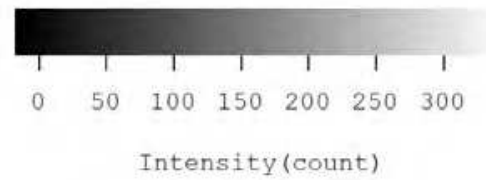





Deviation ( $\mathrm{nm}$ )

Fig. 48. Interference pattern and measured phase map on CCD surface at ambient temperature of 20.71 degrees Celsius. 


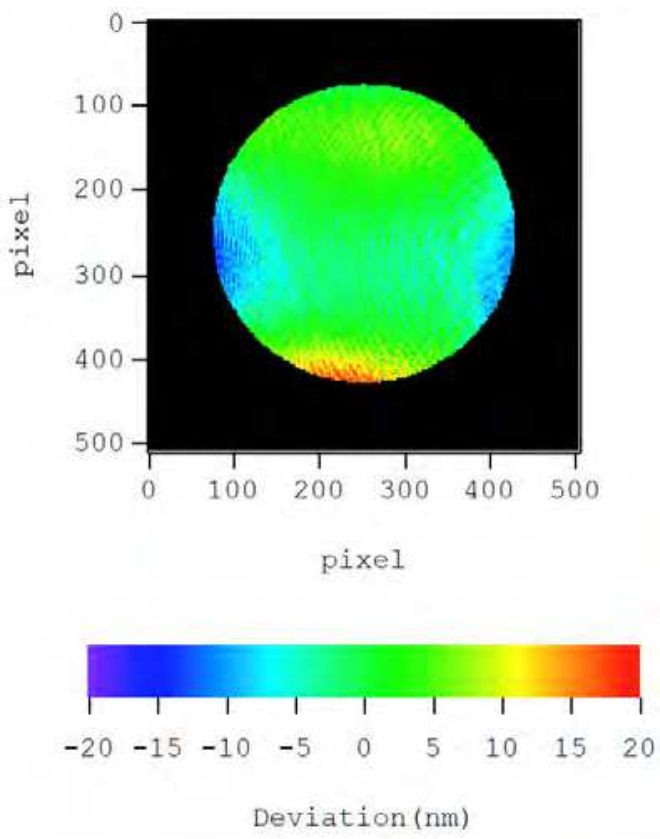

Fig. 49. Relative difference in phase map between fig. 46 and fig. 47.

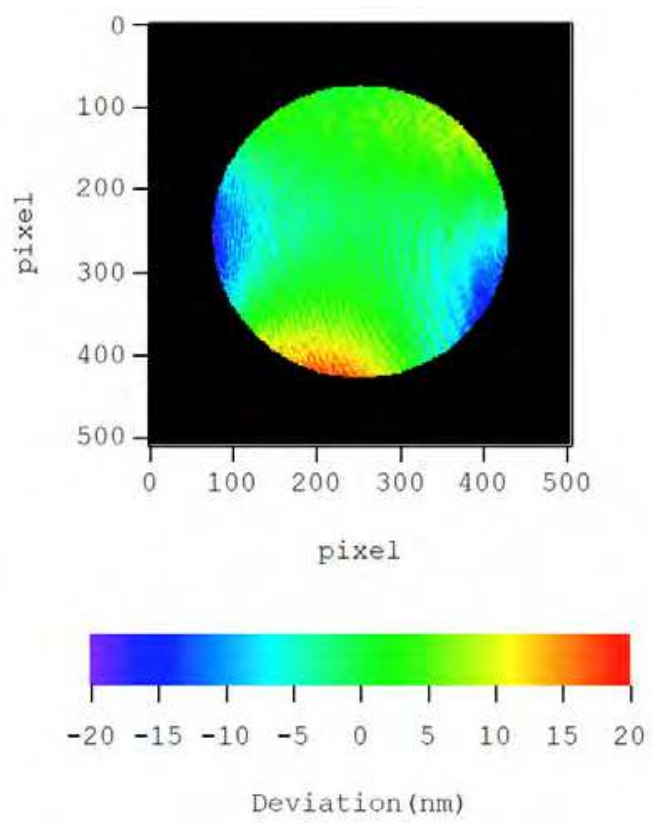

Fig. 50. Relative difference in phase map between fig. 46 and fig. 48 . 

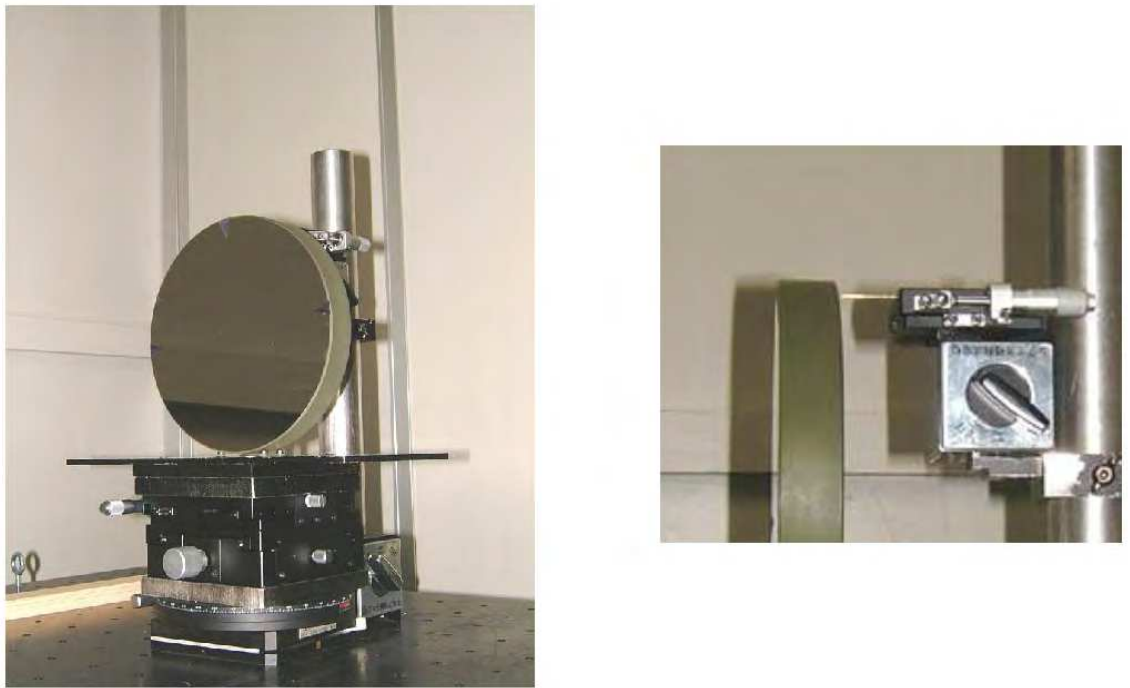

Fig. 51. Mirror supported by three-point suspension of steel balls and single needle.

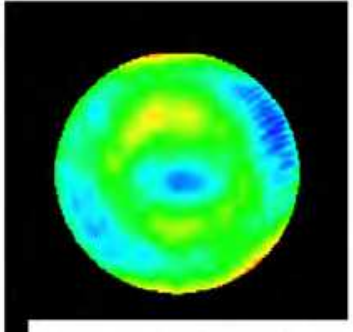

Average from 1st to 10 th

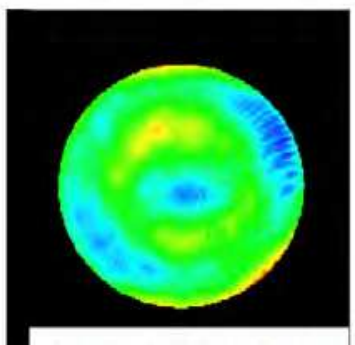

Average from 31 st to 40 th



Average from 11 th to 20 th

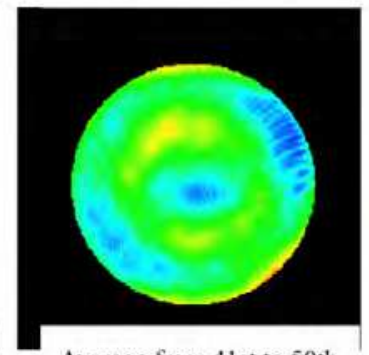

Average from 41 st to 50 th

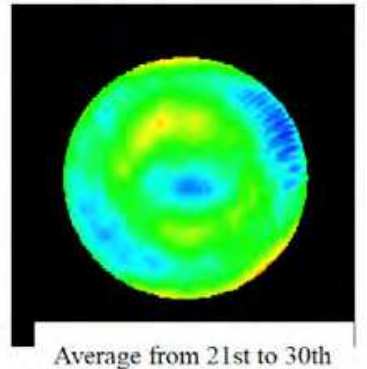

Average from 21 st to 30 th

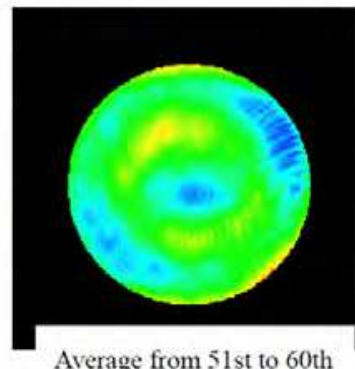

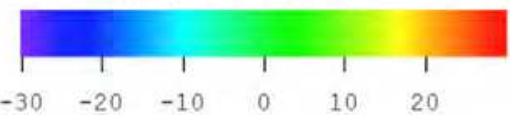

Deviation (nm)

Fig. 52. Averaged images of wavefront for every 10 measurements. 


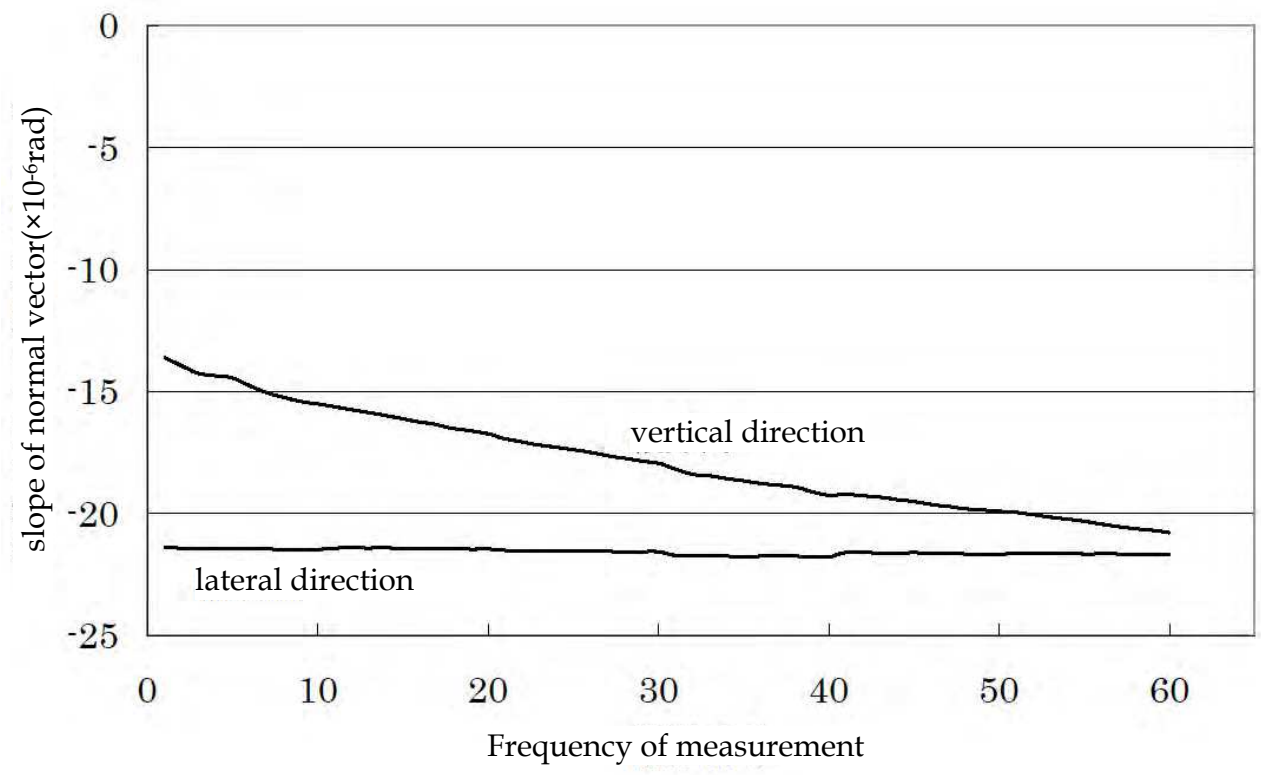

Fig. 53. Temporal change in direction of surface normal vector of test surface.

\section{(1)}
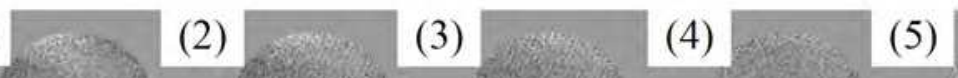

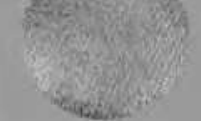

(6)

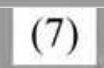

(8)

(9)
(5)
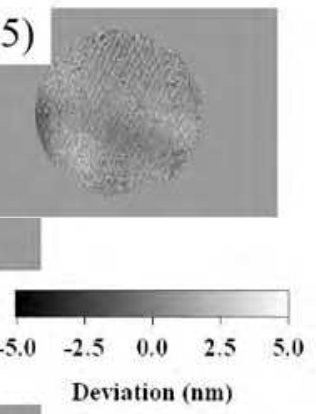

Fig. 54. Differences between ten-measurement average and overall average of 100 measurements. The difference changes slightly due to air current. 


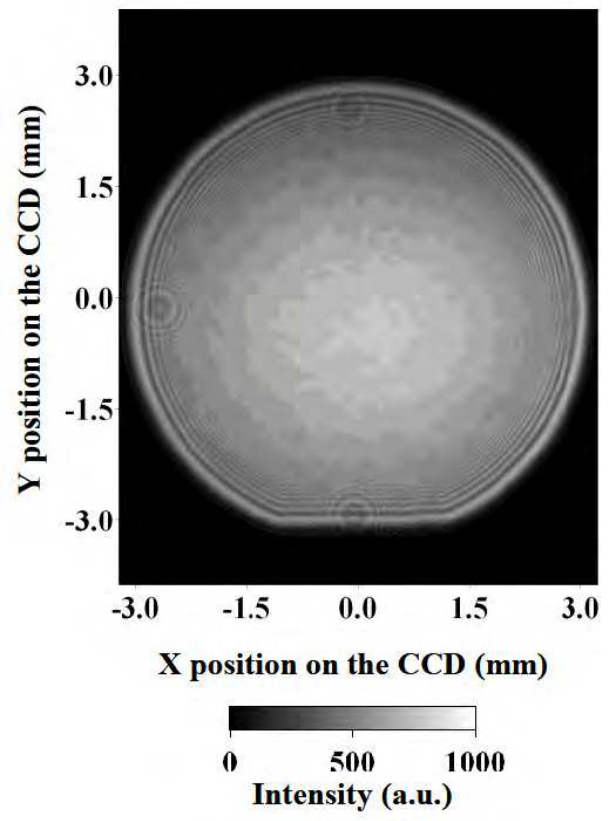

Fig. 55. Light intensity distribution on test mirror surface.

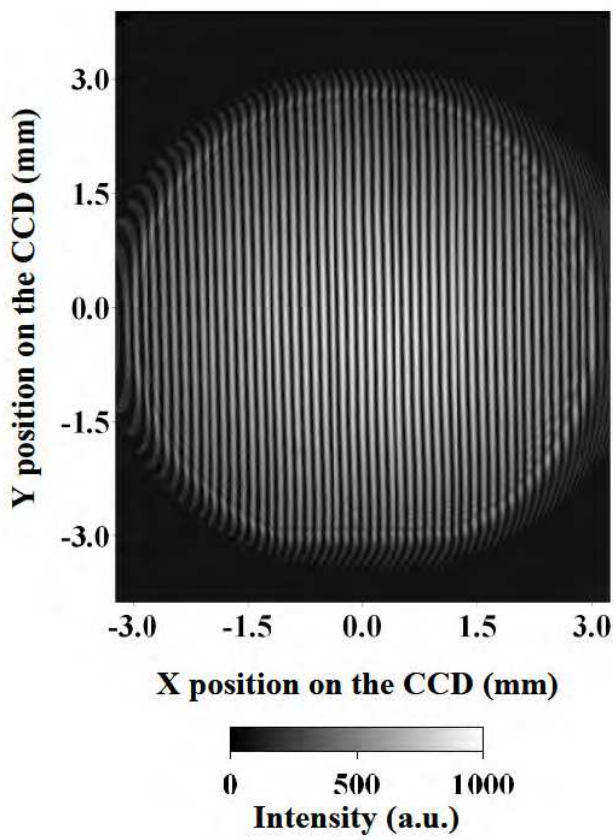

Fig. 56. Typically-observed interference pattern on CCD sensor. 




Fig. 57. Deviation of test wavefront from ideal sphere on CCD surface.

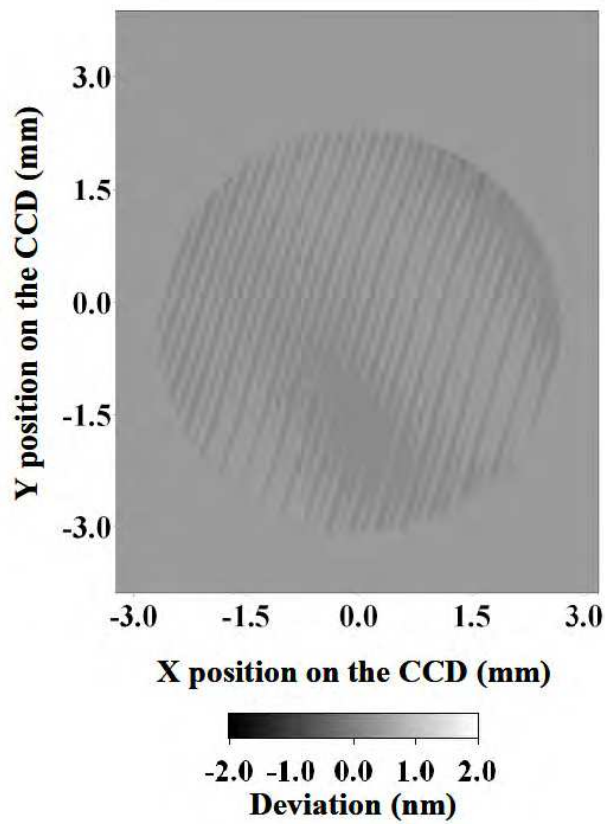

Fig. 58. Difference between averages of first and second 50 measurements. 

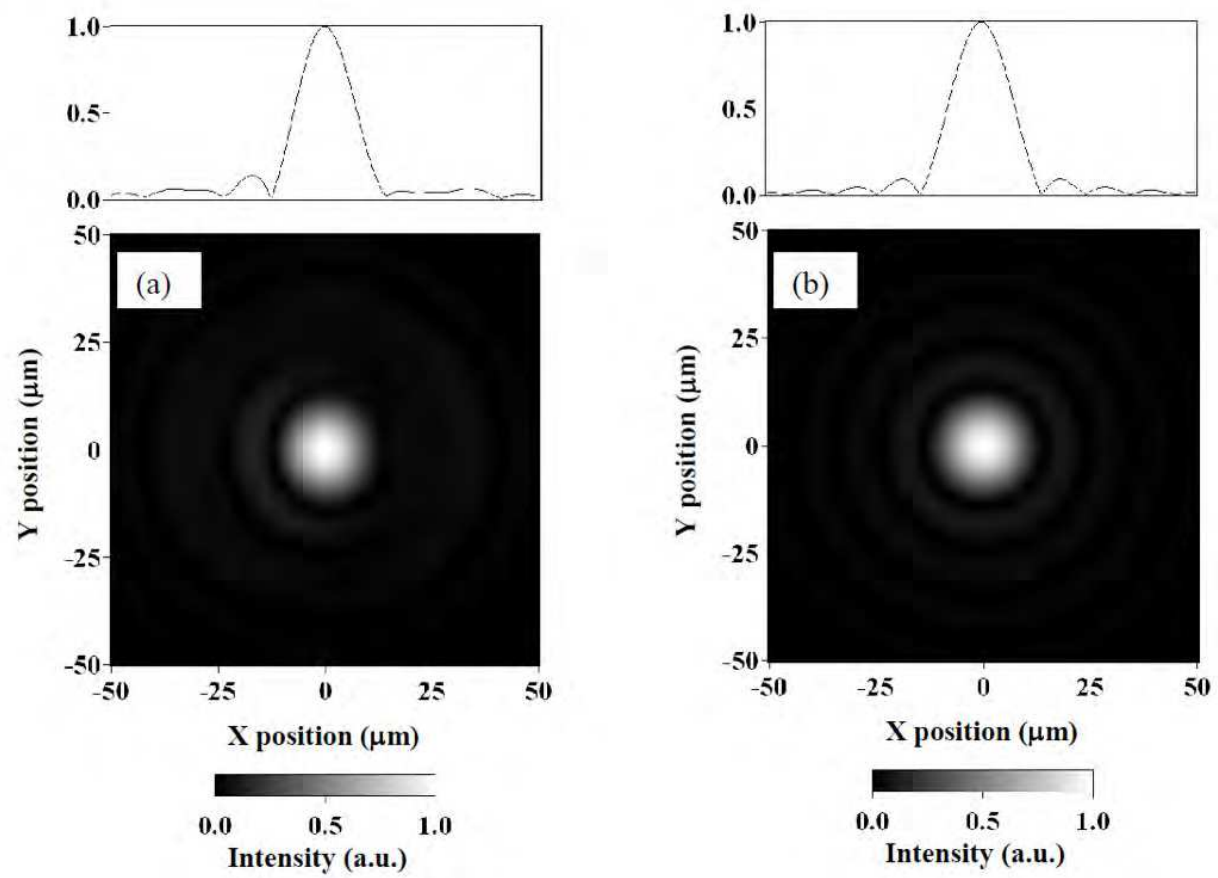

Fig. 59. (a) experimental and (b) ideal intensity map test wave at focal point.
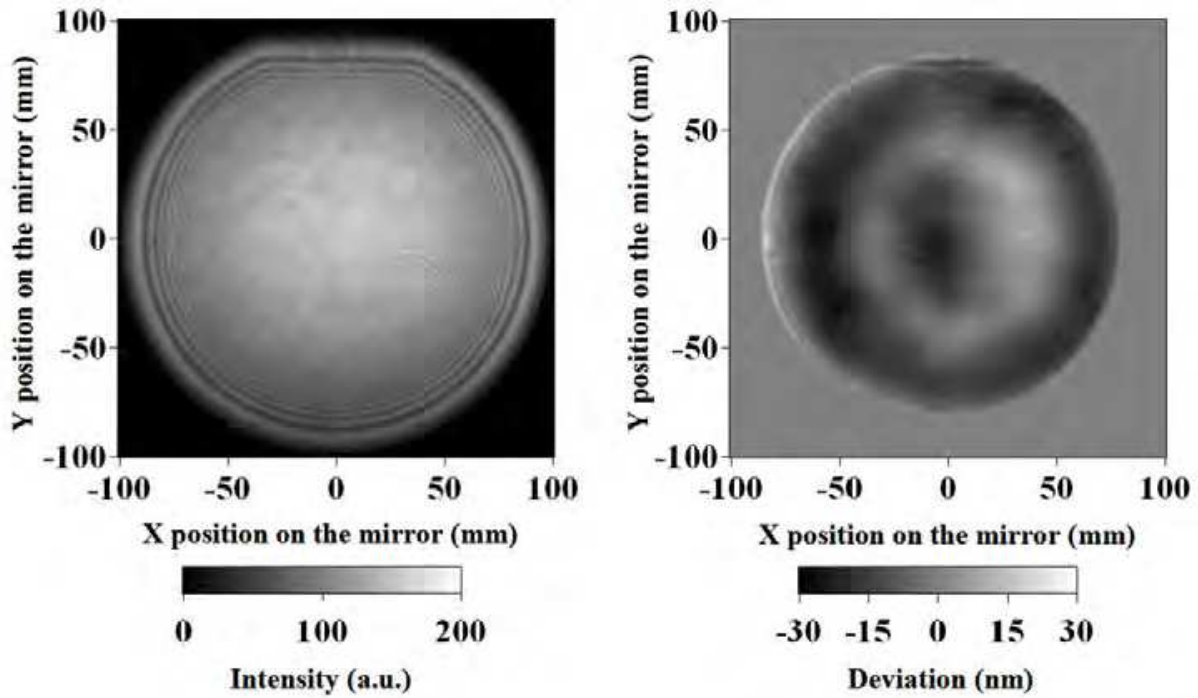

Fig. 60. Reconstructed light intensity map (a) and surface figure (b) on test mirror surface. 


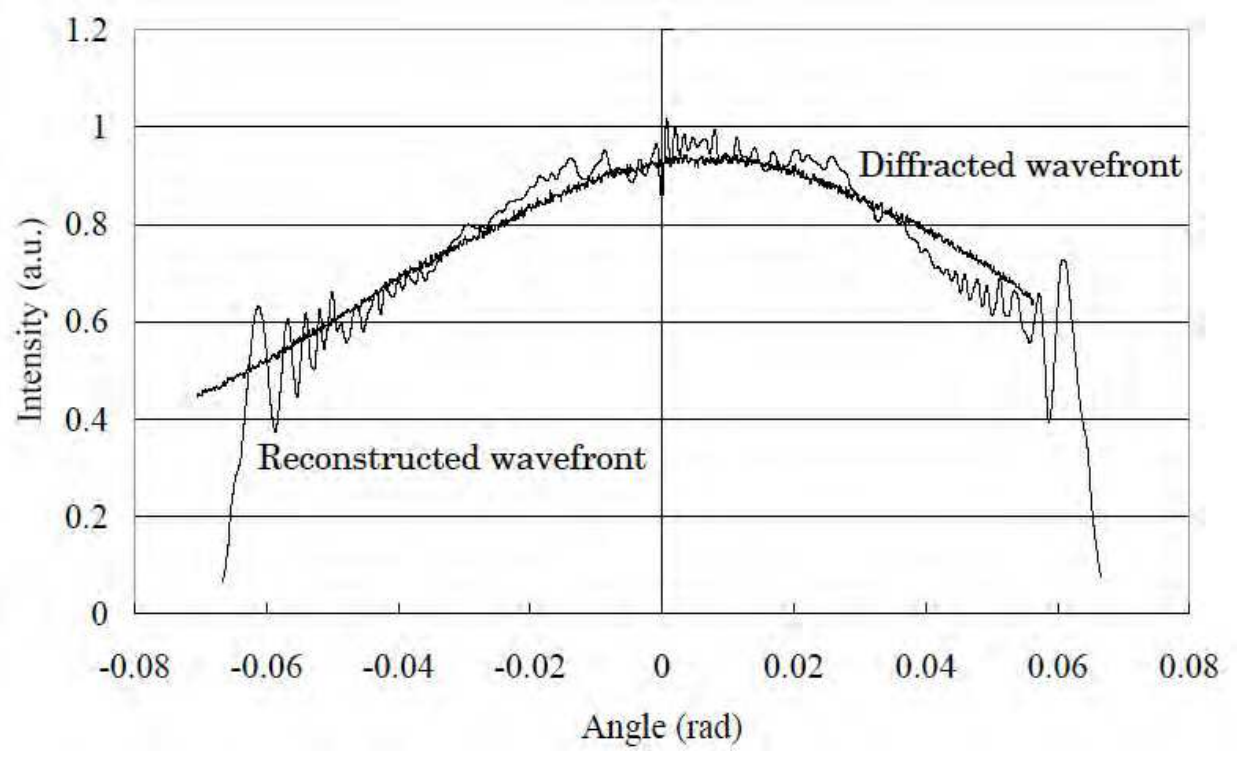

Fig. 61. Comparison of intensity distribution between reconstructed wavefront and fiberoutput.

\subsection{Discussion}

Several measurement datasets are taken to evaluate reproducibility of the PS/PDI measurement without altering the experimental apparatus. After a five-hour warm-up period, the first dataset was recorded, and 30 minutes elapsed before the second data was recorded. The third dataset was measured 6 hours after the first dataset. The difference between the first and second datasets is displayed in fig. 62 on the left, and the difference between the first and third datasets is shown on the right. Although air current noise overlapped with the results in fig. 62, the measurement reproducibility between the first and third datasets is $0.5 \mathrm{~nm} \mathrm{PV}$ and $0.12 \mathrm{~nm}$ rms. In fig. 62, geometrically symmetric patterns are observed, which are caused by numerical error in the reconstruction procedure. In the numerical integration, spatially discretized elements such as the CCD pixels affect the result (Born \& Wolf, 1959). In addition, the reconstructed wavefront on the test mirror surface is also presented in the image discretization, and causes Moire fringes by coupling the numerical error pattern described above (Freischlad \& Koliopoulos, 1990). Improvement of reproducibility in the consecutive measurement requires more warm-up time before the measurement begins. Final evaluation is reproducibility of the measurement results obtained by rotating the test mirror about its optical axis at 90 degrees. The difference between the two results is presented in fig. 63. Here, Moire fringes also degrade the structural coincidence, and the measurement reproducibility is $2.0 \mathrm{~nm}$ PV and $0.85 \mathrm{~nm} \mathrm{rms}$. A more accurate procedure for numerical integration will produce much better results in the future. 

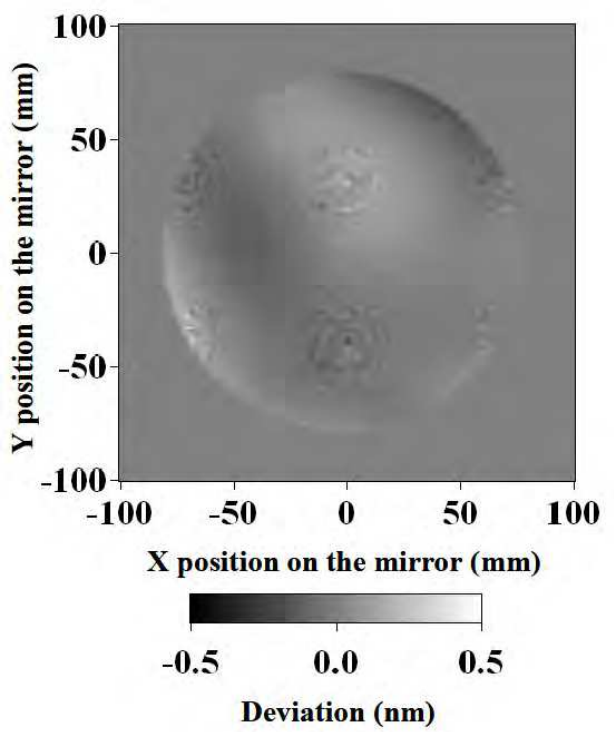

(a)



(b)

Fig. 62. Measurement reproducibility between two datasets taken with interval of 30 minutes (a) and 6 hours (b), respectively.

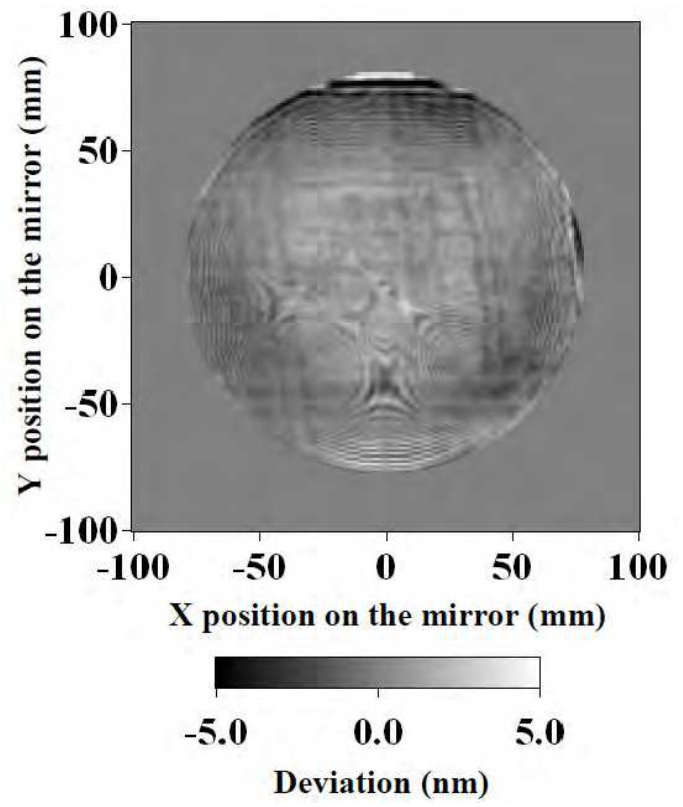

Fig. 63. Difference between before and after rotation of mirror. 


\section{Measurement of aspherical mirrors}

\subsection{Spherical concave mirror with high asphericity}

A highly accurate surface figure is needed not only for a spherical surface but also paraboloidal, ellipsoidal, and flat surfaces. In an ordinary PS/PDI system, a highly aspherical test surface emanates condensed interference fringes on the $\mathrm{CDD}$, and correct recording and analysis of the fringes are not possible. In our PDI system however, location of the two point sources is very flexible, as well as the position of projection plane. Therefore, an appropriate fringe pattern can be obtained for the aspherical surfaces and large-aperture optics. In this last section, trial experiments are conducted to measure an aspherical concave mirror, an off-axis paraboloidal mirror, and a mirror-polished silicon wafer. Fig. 64 shows a photo and schematic of a sample concave mirror having a design value of $1000 \mathrm{~mm}$ ROC. In the beginning, the optical apparatus shown in fig. 6 is used for the measurement. However, the test surface is highly aspherical, and the reflected wave did not converge well. The intensity distribution after reflection on the test surface is shown in fig. 65, and fig. 66 shows the interference pattern between the reference wavefront and the wavefront shown in fig. 65 . These two images are ellipsoidal due to the aspherical test surface. In the ellipsoidal image, the ROC value in the vertical direction is larger than in the horizontal direction. Spatial density of the fringes shown in fig. 66 is an acceptable range for the CCD sensor, and the PS/PDI measurement was implemented. The intensity distribution at the focal point of the test wave is reconstructed in the same manner as described in section 6 and shown in fig. 67 . The point might not be the focal point, leading to the conclusion that the spherical test wave assumption could be incorrect. Another method for determining the location data of the reference fiber end and CCD is necessary. The procedure described in section 4 after the mirror measurement was introduced. In this procedure, only the fiber end for the test wave generation is repositioned to observe the interference pattern caused by two diffraction waves. This data will provide the location data of the fibers and CCD. Here, the low-quality focal pattern shown in fig. 67 could possibly cause degradation of a common path, like the configuration shown in fig. 6 . Hence, reconstruction of the test wavefront is simulated numerically with misalignment of the fiber end for the test wave, and the results are displayed in fig. 68. Even if the test surface is spherical, misalignment of the fiber end results in an imperfect phase map as shown in fig. 68 . Therefore the location coordinate of the fiber end for the test wave must be determined. For this purpose, the concave mirror measurement in section 6 is applied to this experiment. When the fiber end for the test wave is positioned at the center of curvature of the spherical concave mirror, light reflected by the mirror focus on the fiber end again, and movement of the fiber end and the focal point are symmetrical about the center of curvature. The movement of the fiber end and location of focal point, which is determined after the mirror measurement, location coordinate of the fiber end for the test wave is determined. In fig. 69, the measurement procedures mentioned above are shown. In step (1), the interference pattern caused by two diffraction waves is measured, and locations of both the reference fiber end and CCD sensor are determined and fixed. In step (2), considering the measurement of concave mirror described in section 6, the focal point or reference fiber end is determined. In step (3), measurement of the test mirror shown in fig. 64 is conducted and the data is analyzed with data obtained in (1) and (2). Fig. 70 shows the resultant test wavefront on the CCD. In 100 consecutive measurements, several datasets exhibit an interesting behavior, an example of which is shown in fig. 71. This may be caused by overreaction of the unwrap algorithm for phase calculation, and such a phenomenon is induced by mechanical vibration or considerable 
change in the interference pattern during measurement. Excluding the invalid data, the averaged wavefront data shown in fig. 70 is calculated from 94 datasets. Next, the surface figure of the mirror is reconstructed using fig. 65 and fig. 70, and the results are shown in fig. 72. The left image of Fig. 72 confirms that the test mirror is a circle, and the diameter is estimated to be $109.2 \mathrm{~mm}$ by referring to the three markers on the outer edge. The surface shape appears to be ellipsoidal, which is consistent with fig. 65. Fig. 72(left) is compared with the actual intensity distribution of the diffraction wave, and they agree well as shown in fig. 73 . Fig. 74 shows the measurement reproducibility of the sample rotation evaluated using the same method used to produce fig. 63. The reproducibility is $5 \mathrm{~nm} \mathrm{PV}$ and $1.2 \mathrm{~nm}$ rms. The test mirror has asphericity of $10 \mu \mathrm{m}$, and our PS/PDI system exhibited a successful measuring capability for such a highly aspherical surface.
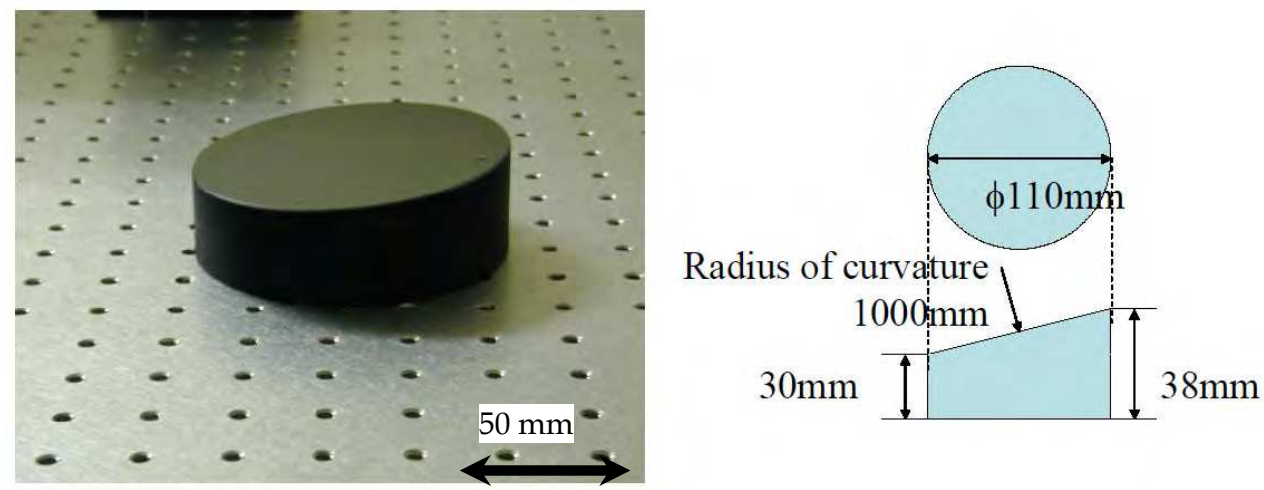

Fig. 64. Photo and drawing of aspherical concave mirror measured in this section.

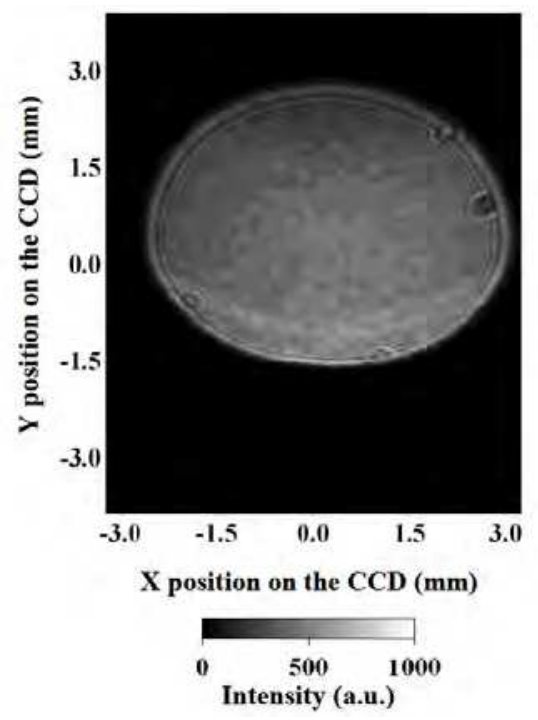

Fig. 65. Intensity distribution of reflected test wavefront on CCD. 


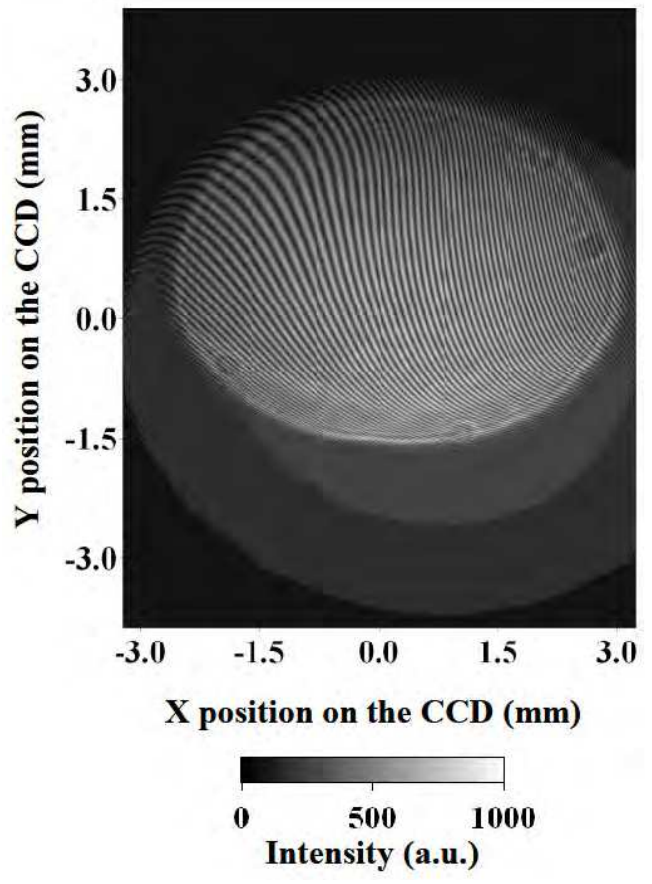

Fig. 66. Typical interference pattern taken at CCD surface.

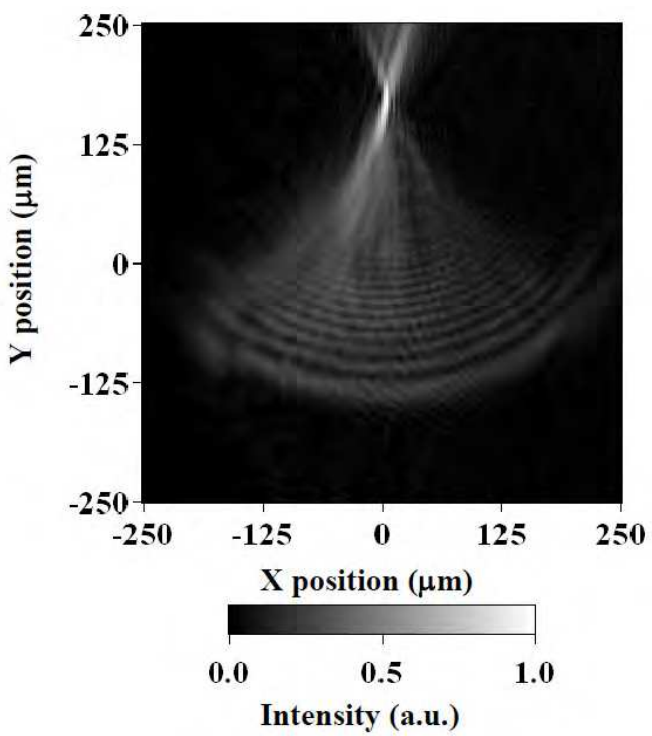

Fig. 67. Reconstructed intensity distribution at focal point of test wave. 

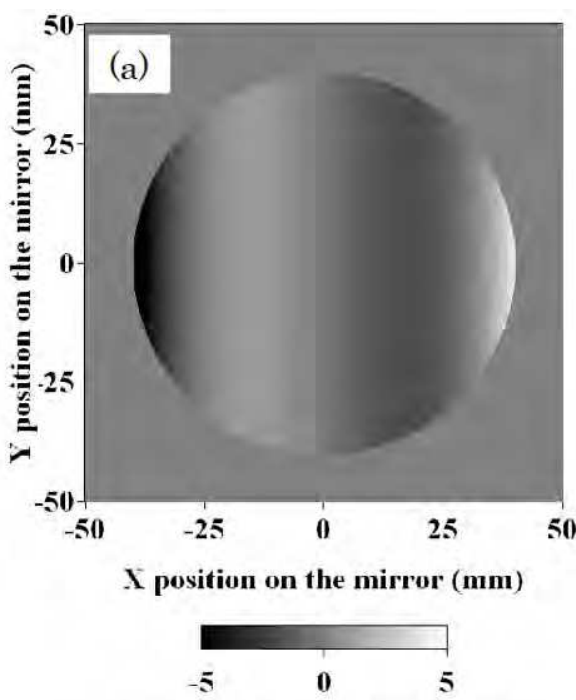

Deviation on the mirror $(\mathrm{nm})$
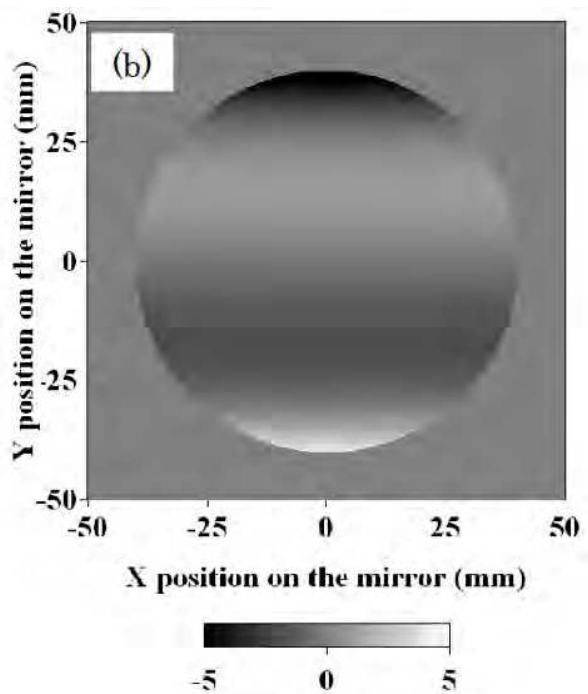

Deviation (nm)


Fig. 68. Numerical simulation of error in phase map caused by misalignment of fiber end at (a) $x=1 \mathrm{~mm}$, (b) $y=1 \mathrm{~mm}$, and (c) $\mathrm{z}=50 \mu \mathrm{m}$. 




Fig. 69. Three step measurement for highly aspherical concave mirror.

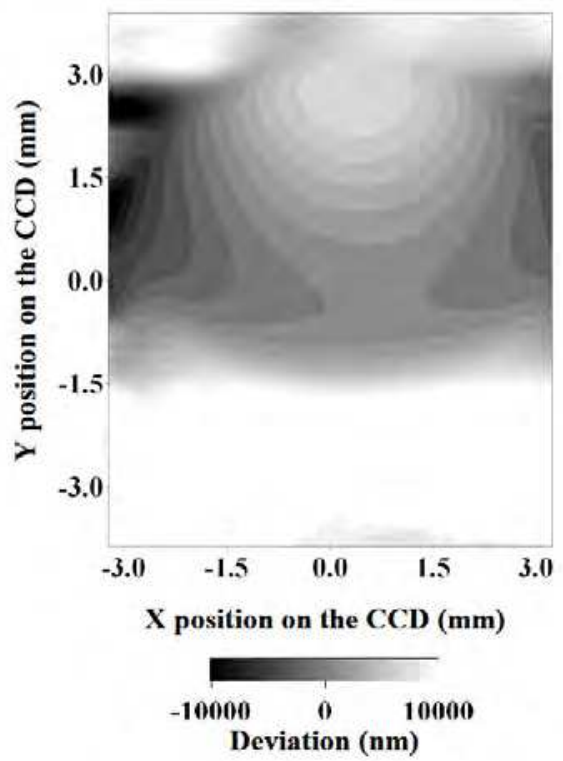

Fig. 70. Wave front deviation of test wavefrom ideal sphere on CCD surface. 


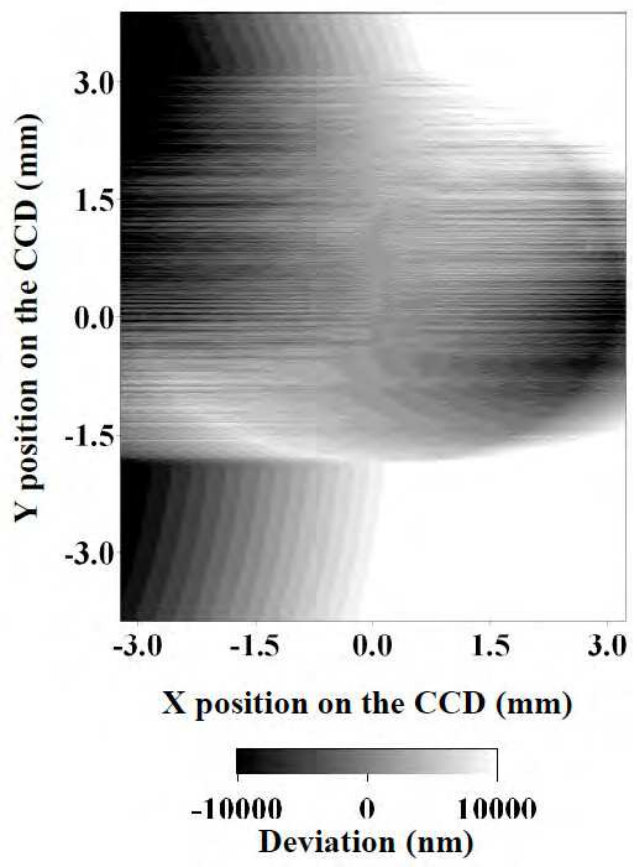

Fig. 71. Example of vibration-induced phase error enhanced by unwrap process.
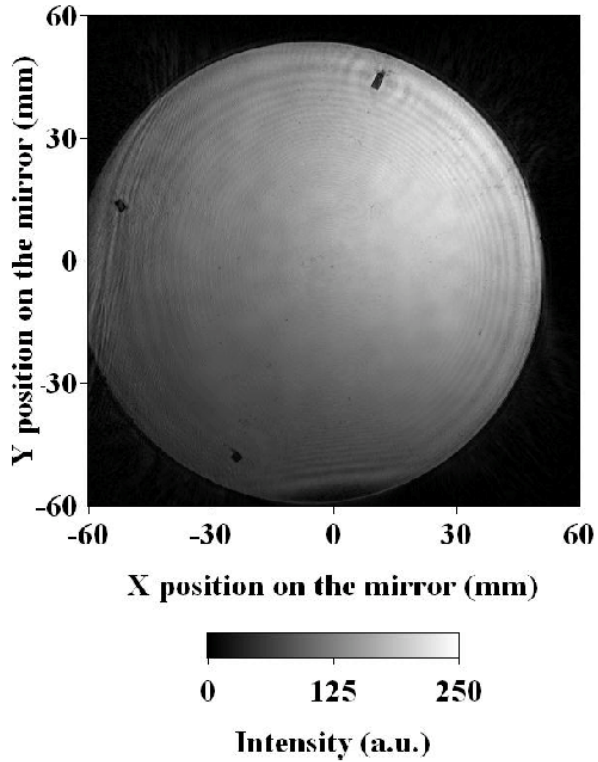

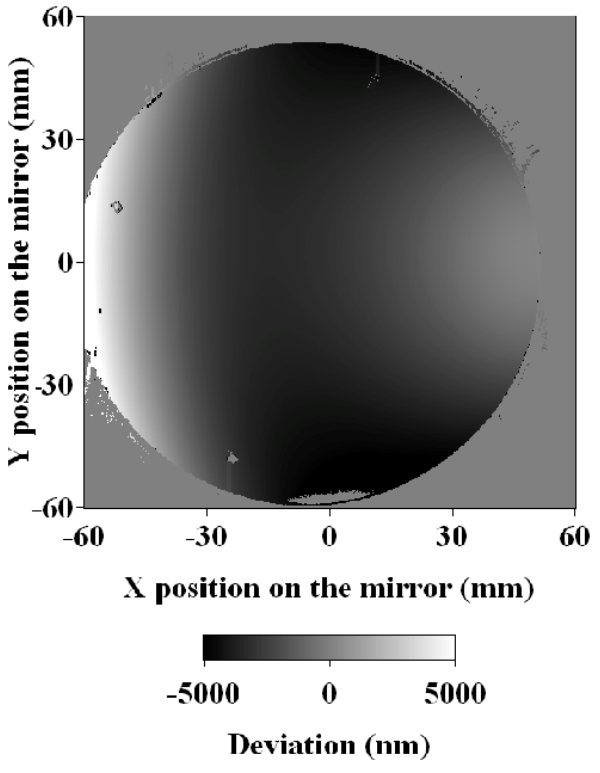

Fig. 72. Reconstructed light intensity distribution (a) and surface figure (b). 




Fig. 73. Comparison of intensity distribution between reconstructed wavefront and fiberoutput.
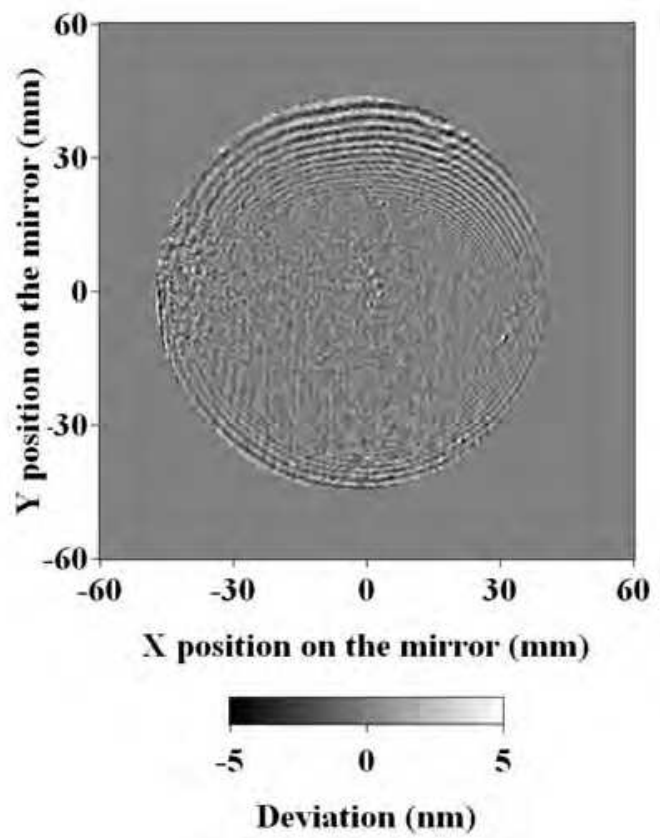

Fig. 74. Difference between before and after rotation of mirror. 


\subsection{Off-axis parabolic mirror}

The alternative off-axis parabolic mirror shown in fig. 75 is also measured. The mirror has a $360 \mathrm{~mm}$ outer diameter and a $258 \mathrm{~mm}$ inner diameter, and the mirror surface has a cross section defined by the equation of $y=x^{2} / 4 f(f=75 \mathrm{~mm}, 258 \mathrm{~mm}<x<360 \mathrm{~mm})$. This mirror is designed to focus many laser beams onto a single point, and cannot converge the diffraction wave from a fiber end. Hence, the optical configuration shown in fig. 6 is no longer valid, and the mirror measurement is performed with the alternative set up presented in fig. 76 . The test wavefront is converted to a plane wave by positioning the fiber end to the focal point of the mirror. Appropriate positioning of the reference fiber end causes generation of a usable interference pattern on the CCD surface. The wavefront of the reference wave converts to a plane wave through a relatively long propagation distance, and the resultant interference pattern is not very dense. The position of the test fiber end is adjusted in accordance with fig. 77. Namely, the fiber end is moved to the actual focal point of the mirror which is illuminated by the plane light wave. Fig. 78 shows the data from the optical power meter, which depends on the three dimensional position of the fiber end. Changes in these curves are used to determine a position of fiber end accurately. The interference pattern recorded by the optical configuration of fig. 78 is shown in fig. 79 , where distance between the test mirror and CCD is $1 \mathrm{~m}$. The resultant reconstructed wavefront is shown as the deviation from a flat surface in fig. 80. In the numerical process, locations of the reference fiber end and CCD are determined by relying on the situation of interference between the plane wave and the spherical wave on the CCD surface. Thus, the interference patter is coaxial. In fig. 80, vertical bleary stripes correspond to tool marks scratched during the cutting of the mirror surface. Reproducibility of the data shown in fig. 80 for 30 minutes is evaluated and displayed in fig. 81 , and it is $10 \mathrm{~nm}$ PV and $1.3 \mathrm{~nm}$ rms. Fig. 81 has a vibration-induced coaxial error pattern at double the frequency of the interference pattern due to the vacuum system supporting the mirror. Finally, influence of the misalignment of the test wave fiber is studied. In the measurement shown in fig. 76, the PS/PDI data provides the distribution of the relative optical path differences in the collimated beam from the test mirror. The optical path distribution must be subtracted from data such as the data in fig. 80. In fig. 82, the upper three images display the distribution of relative optical path difference caused by misalignment of the test wave fiber end in three directions, and the lower three images display the consequent deformation of the wavefront from a flat profile. When the fiber end is moved $120 \mu \mathrm{m}$ in the Y-direction, the result is shown in fig. 83 and is consistent with the lower middle image in fig. 82 .

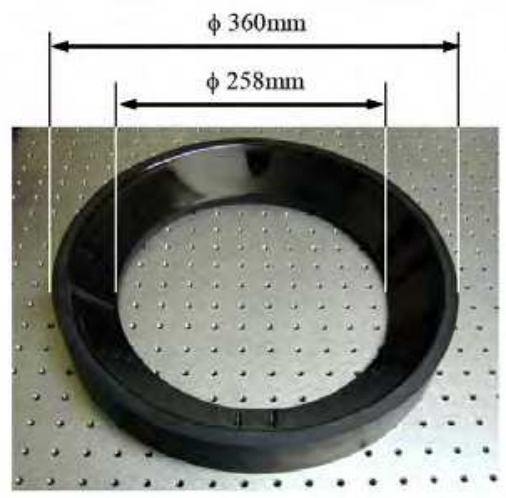

Fig. 75. Photo of aspherical mirror having off-axis paraboloid of revolution. 


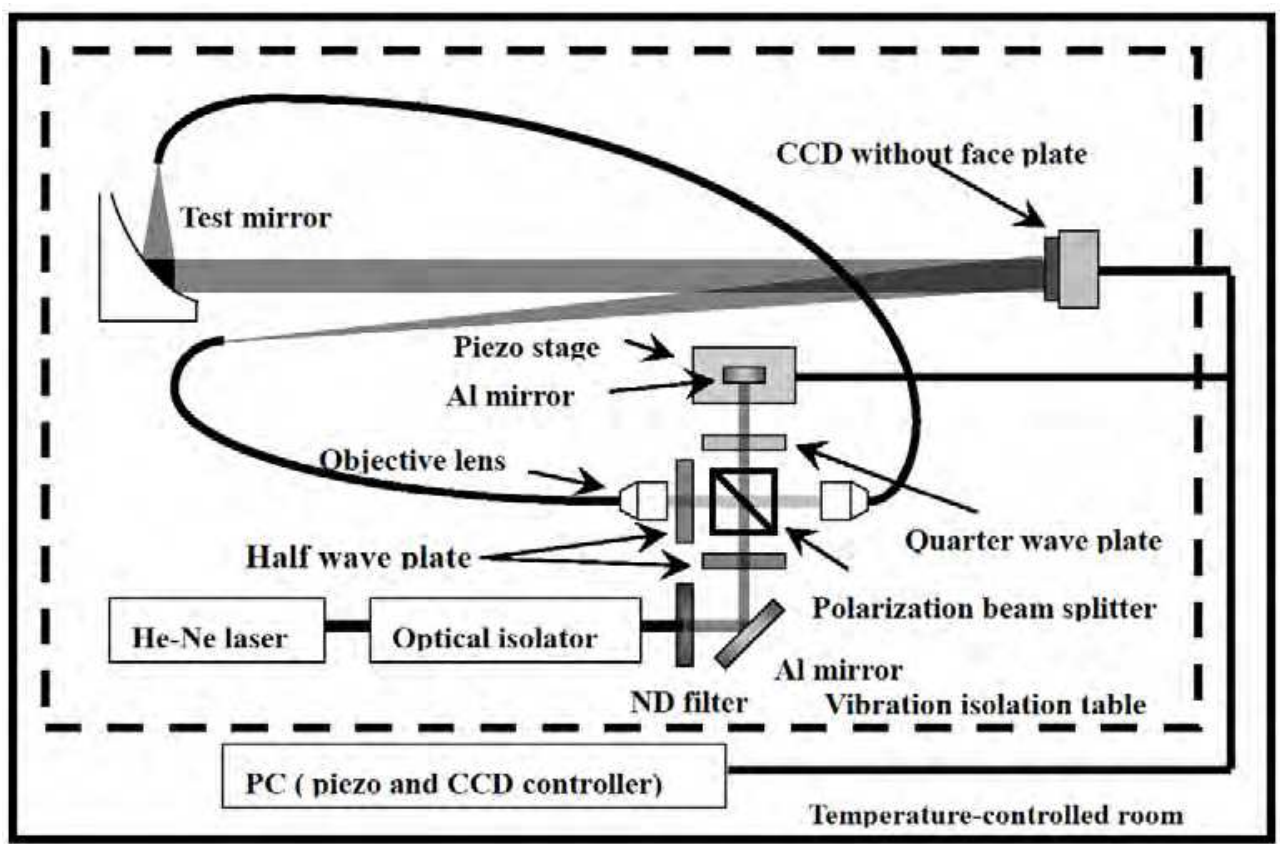

Fig. 76. Optical configuration of the PS/PDI system for off-axis parabolic mirror.



Fig. 77. Installation of optical fiber end to focal point of test mirror. 


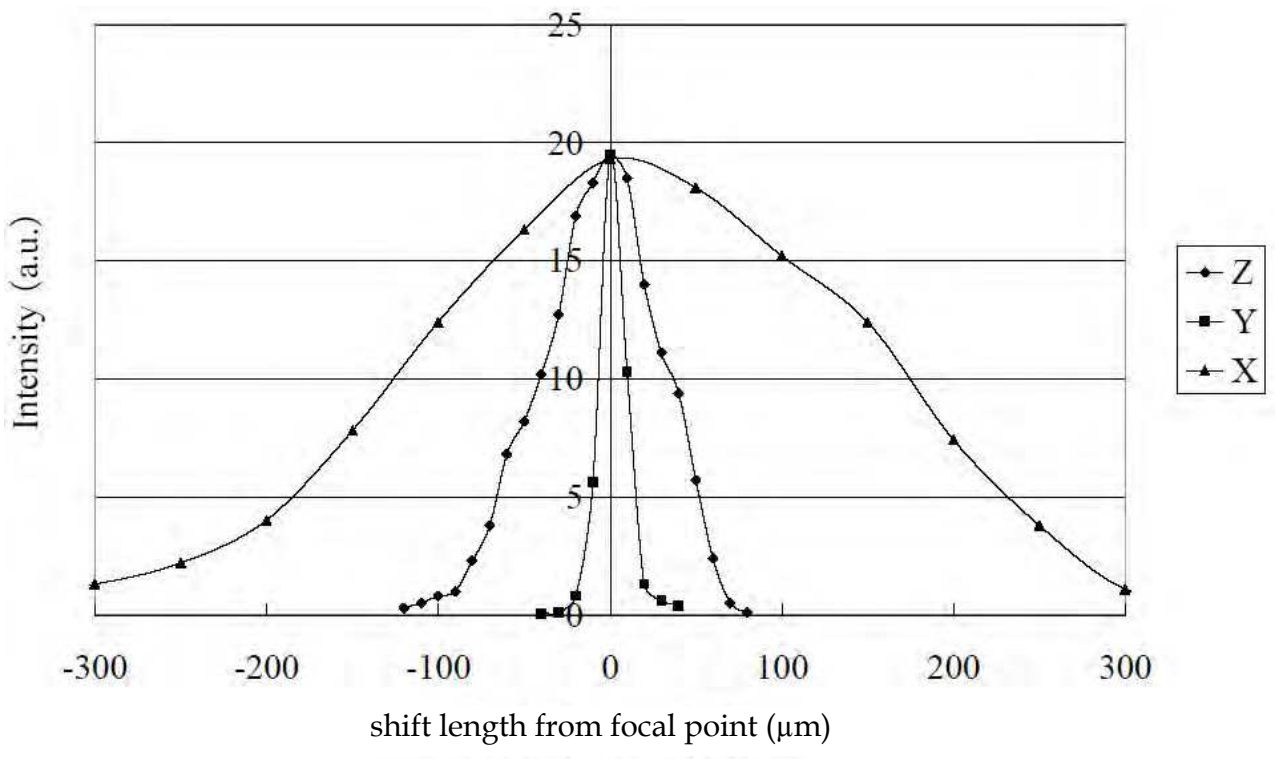

Fig. 78. Output of optical power meter, as indicated in fig. 77 , is sensitive enough to locate fiber end for alignment.

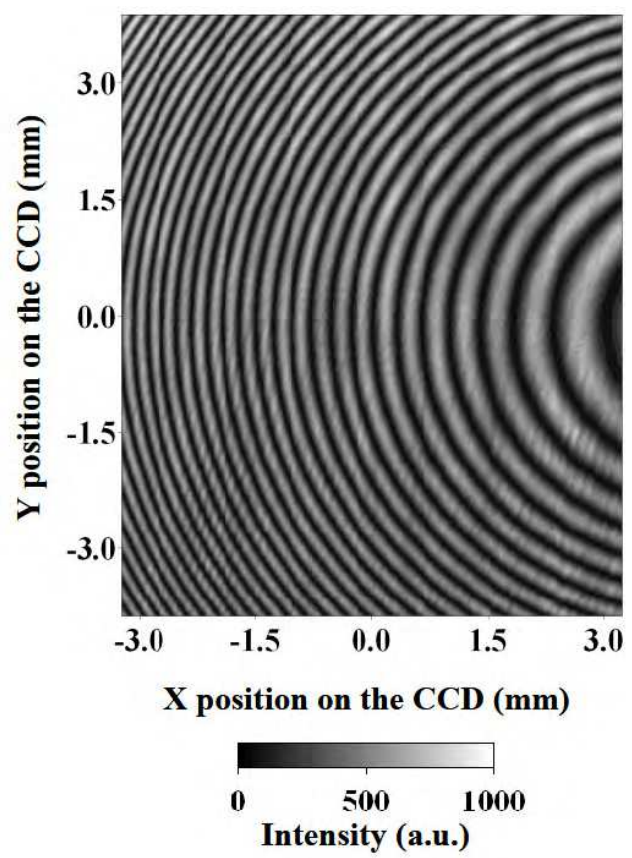

Fig. 79. Typical interference pattern recorded in measurement of the parabolic mirror. 


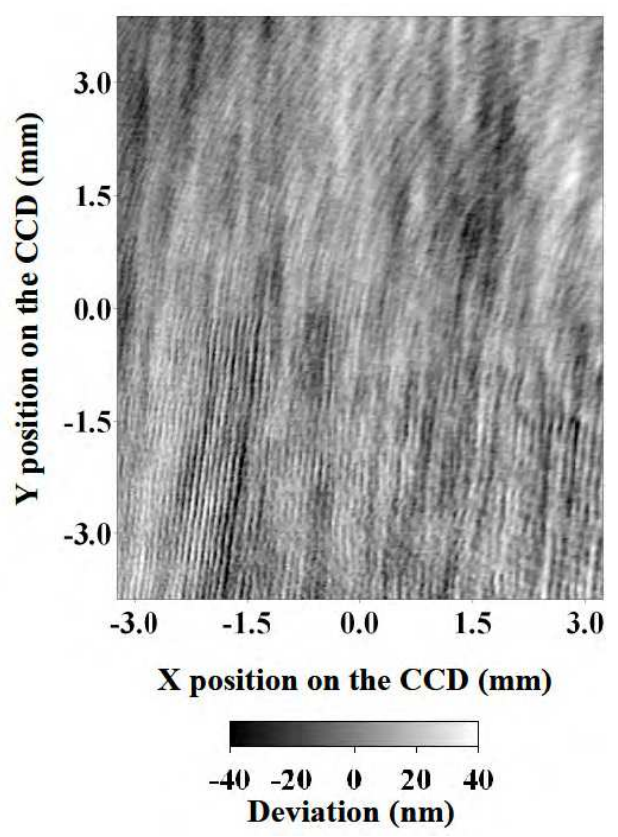

Fig. 80. Deviation of reconstructed surface figure from flat surface.

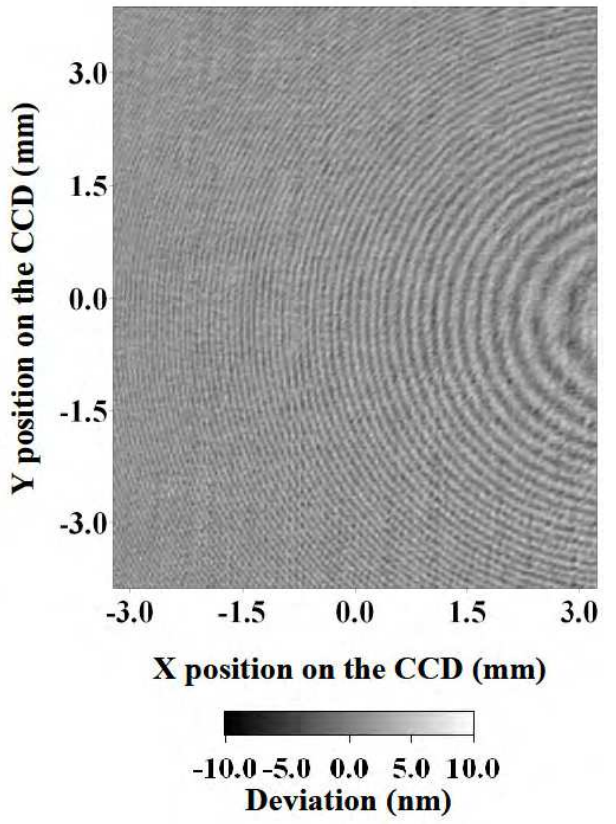

Fig. 81. Reproducibility of the measured wavefront shown in fig. 80 . 


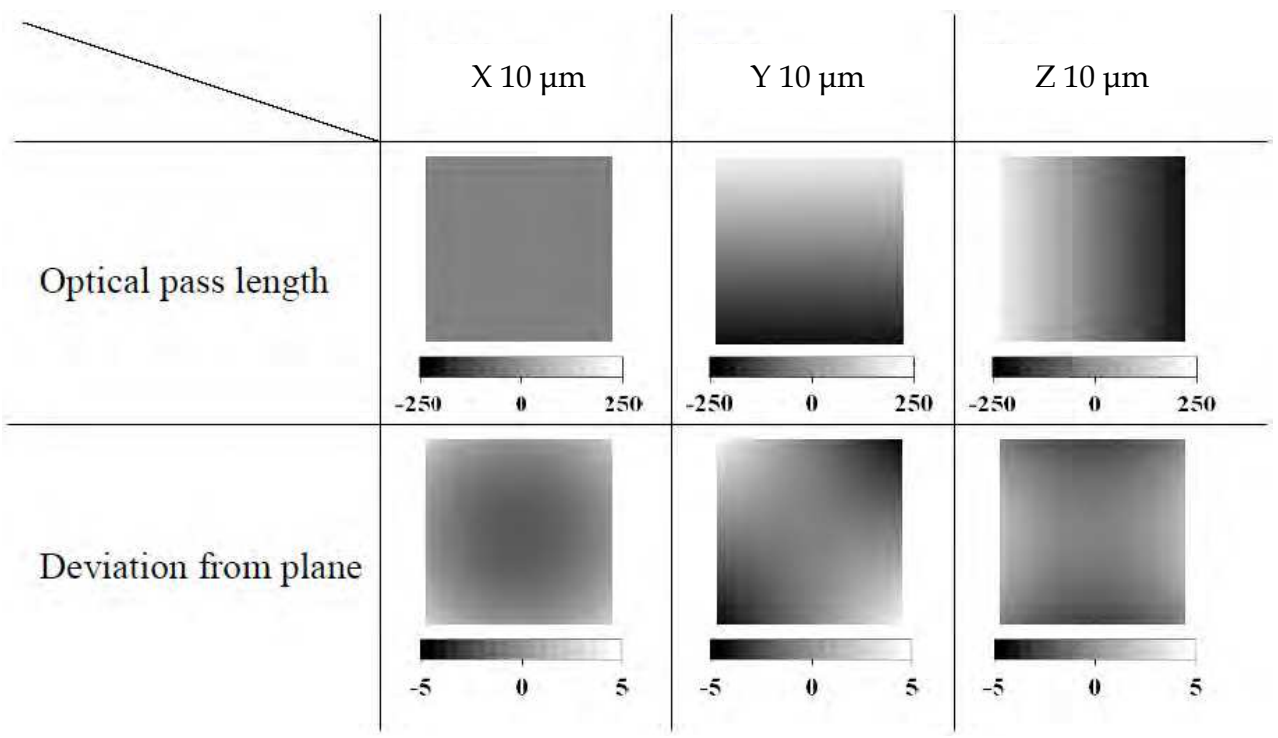

Fig. 82. Numerically simulated measurement error induced by misalignment of fiber end in the parabolic mirror experiment.

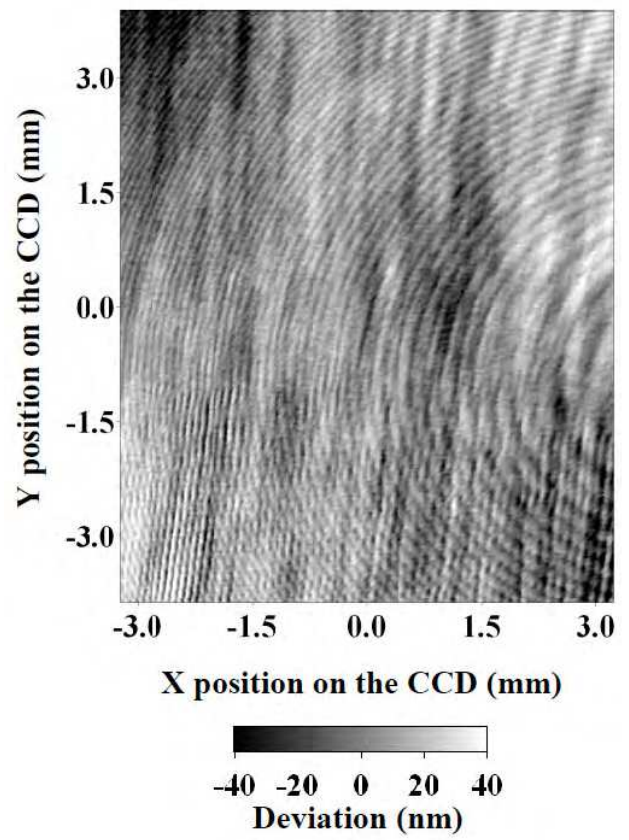

Fig. 83. Deviation of reconstructed wavefront from flat surface in condition of $120 \mu \mathrm{m}$ misalignment of fiber end for test wave front. 


\subsection{Measurement of silicon wafer as flat surface}

Measurement of a flat surface may be possible using our PDI system only. By positioning the two fiber ends properly, a usable interference pattern is recorded by the optical configuration shown in fig. 84. The flat test mirror is placed in front of the CCD obliquely, and the surface normal is at 45 degrees to the surface normal of the CCD. The test wave reflected by the test mirror includes information of the test surface figure, and an interference pattern could be generated by the reference wave as shown in fig. 84. Fig. 85 shows the typical interference pattern, and the reconstructed wavefront, which is the deviation from an ideal sphere, is displayed in fig. 86. The wavefront data is converted to the surface figure of the silicon wafer using the ray trace method and is shown in fig. 87 . The surface figure is also measured by a white-light interferometer as shown in fig. 88 . The datasets agree well in the low-frequency components.



Fig. 84. Optical configuration of the PS/PDI system for measurement of silicon wafer surface. 




Fig. 85. Typically observed interference pattern in measurement of silicon wafer.

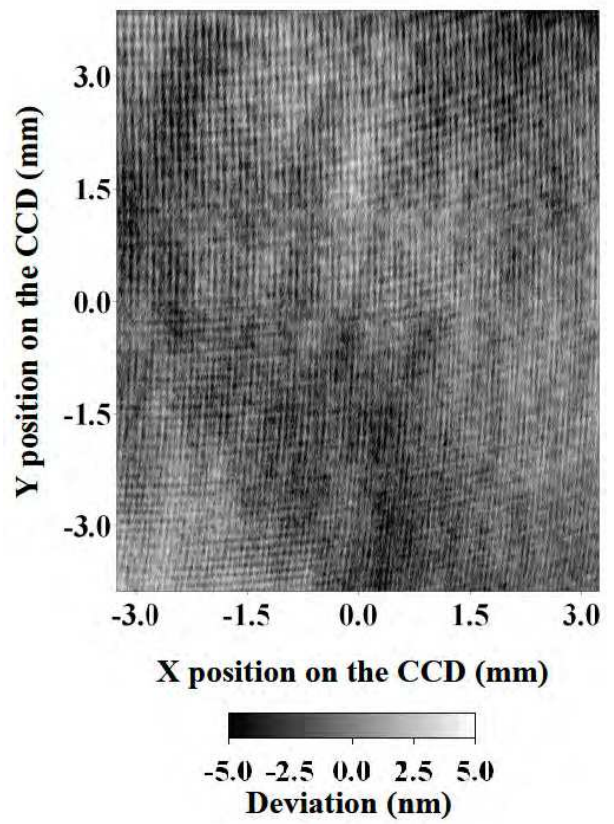

Fig. 86. Deviation of measured wavefront on CCD from ideal sphere. 


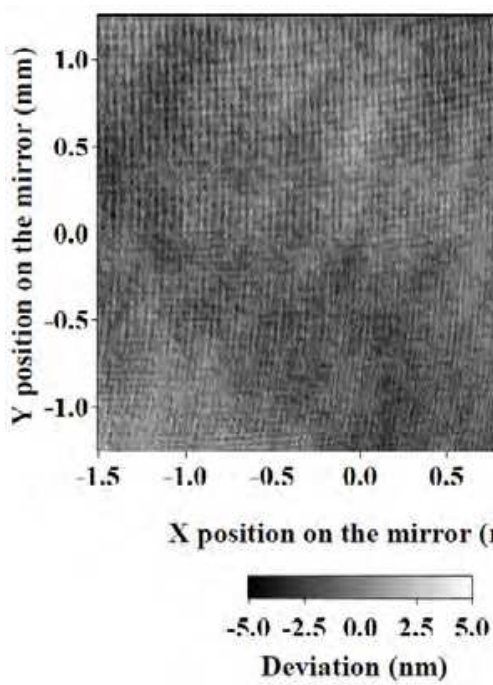

Fig. 87. Numerically derived surface figure of silicon wafer by ray trace.

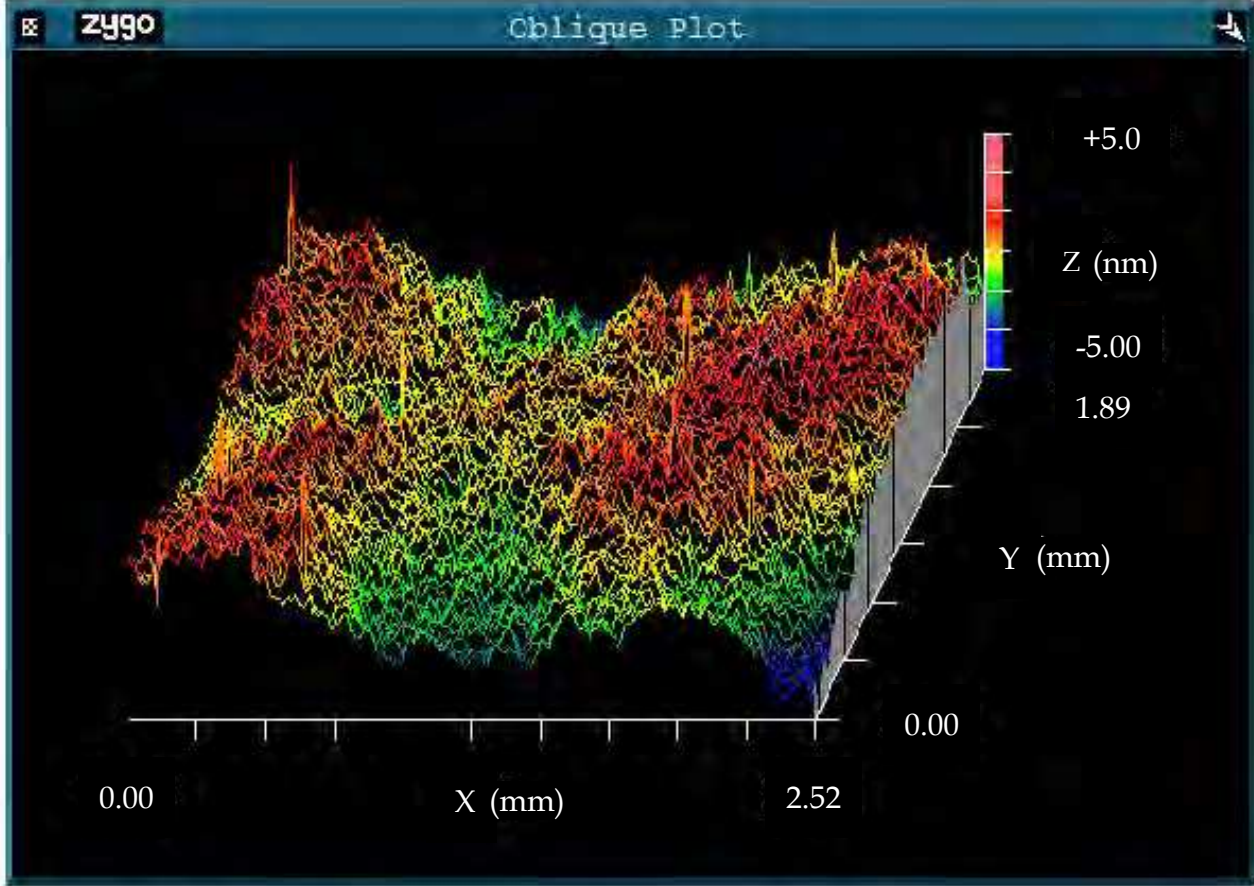

Fig. 88. Surface figure of the silicon wafer measured by commercial white light interferometer. 


\section{Conclusion}

In this chapter, known issues in previously studied PDI optics are explained and a novel PDI optical system is proposed for ultra-precise measurement of absolute surface figure for large-aperture optics. The proposed PS/PDI optics system is developed with two singlemode optical fibers. Details of both the apparatus and analysis of interference fringe are explained. In the previous PDI system, a single diffraction wave from a tiny aperture acts as both the test wave and reference wave, and half of the wavefront is used for surface figure measurement. A new configuration was developed for the interferometry using two small apertures as origins of diffraction waves, where the apertures are ends of single-mode optical fibers. These fibers are pointed in opposite directions. Hence, the entire wavefront will be used for surface figure measurement. The two fibers must be close to each other to realize common-path interferometry. Therefore, fiber ends are sharpened by chemical etching and the apexes are polished obliquely for optimization of emission directions. To obtain highly spherical diffraction waves, the polishing angle is carefully determined by numerical simulation based on the Fresnel-Kirchhoff integral. In the measurement process, the CCD camera, which records the interference pattern, is synchronized to a PZT stage, which controls the relative optical phase by changing the length of the optical path, to implement the phase-shift algorithm. The entire control procedure of the interferometer is studied and optimized.

Measurement accuracy of the PS/PDI system is evaluated experimentally by the interference of two diffraction waves. Namely, in the PS/PDI system, two optical fiber ends emit diffraction waves, and these ends are capable of generating an interference pattern when they are oriented in the same direction. The two diffraction waves having highly spherical wavefronts allow estimation of measurement accuracy of the interferometry system. An interference pattern generated by two fiber ends has several tilt fringes due to the optical configuration between the fibers and CCD camera. Such fringes are analyzed numerically by a phase-shift algorithm, and the resultant phase map is unwraped to obtain a continuous initial phase map. In the initial phase map, location information of both fibers and the CCD is included. We described an analytical method to find their positions from the initial phase map. By using the least-squares method, their spatial configuration can be determined accurately due to interference between two ideal spherical waves. In the PS/PDI system, phase-shifting interference patterns must finally be generated on a CCD surface for digital recording. For the purpose of forming the interference pattern on a $C C D$, an imaging lens is not acceptable due to optical aberrations. A fiber optic window (FOW) serving as the projection plane for the interference pattern is carefully examined. Several types of measurement errors, including the FOW-induced disturbance in the interferogram, are considered. The FOW-induced error is highly reproducible and can be eliminated by subtracting one dataset from another. Random error caused by mechanical vibration is eliminated by averaging many datasets. Temporal fluctuation of laser light is reduced with a long warm-up time for the system. Although the FOW error is suppressed, its residual quantity is not negligible for the sub-nm accuracy of the PS/PDI. The latest PS/PDI system accepts direct projection of an interferogram on a special CCD camera without any projection plane or lens. This particular CCD camera has no protection plate, and the bare 
CCD sensor records the interference pattern directly without any unwanted stray light or local interference. As a result, measurement accuracy of the PS/PDI system is estimated to be $0.7 \mathrm{~nm}$ PV and $0.07 \mathrm{~nm}$ rms for mirror measurement.

This chapter clearly describes the necessity of a wavefront reconstruction process for PS/PDI measurement of aspherical or non-ideal mirrors. The explicit result of PS/PDI or phase map on the CCD sensor must be processed numerically for precise reconstruction of test mirror surface figure. Since wavefront propagation is developed by numerical simulation based on the Kirchhoff-Helmholtz integral theorem, interference between two diffraction waves emitted from optical fiber ends is analyzed. Assuming PS/PDI measurement of an ideal spherical concave mirror, the wavefront analysis returns highly important results. That is to say, a phase map, which can be derived at the CCD surface, depends on light intensity distribution on the test mirror surface, but such a phase map can be transformed to an exact surface figure through the wavefront reconstruction process. In the case of an aspherical mirror having high a surface figure error at a high spatial frequency, the phase map on the CCD surface is very different from the real surface shape. For such a situation, the wavefront reconstruction procedure is quite effective. In the case of an aspherical mirror with low-frequency surface error, the reflected wavefront does not have considerable distortion during its propagation because the propagation distance is not as long. Of course, the wavefront reconstruction process results in much better surface figure data. Finally, the wavefront reconstruction process based on the $\mathrm{KH}$ integral will produce an absolute surface figure of the test mirror with the transformation of phase map measured on the CCD sensor serving as a projection plane.

This study discussed the PS/PDI measurement of a nearly ideal spherical concave mirror, and highly precise measurement of the absolute surface figure with sub-nanometer accuracy is confirmed in a series of experimental measurements. In the measurement of the mirror surface, the support method for the mirror is a critical issue. We adopted a three-point support using small steels balls combined with a supporting needle. Although tilt of the mirror surface is induced by thermal expansion of the needle, considerable deformation of the mirror surface is not observed. These mirror fixing techniques are the best options at present. From 100 consecutive measurement results, reproducibility of the results is evaluated with two averaged datasets derived from the first and second 50 datasets. The evaluated value is worse than that estimated in section 4 . This is caused mainly by air current between the optical fiber end and the test mirror, and such a considerable influence can be reduced by averaging many datasets. Many tilt fringes exist in the actual mirror measurement even with an ideal optical set up, which must be analyzed to determine location data of the reference fiber end and CCD sensor. Since the test mirror is a nearly ideal spherical, the location data is derived by assuming that the test wave is still highly spherical after reflection. As a result, the surface figure of the test mirror is reconstructed precisely and without issues in accordance with the instruction described in section 4 . With two data results recorded at a six hour interval, the measurement reproducibility is estimated to be $0.5 \mathrm{~nm}$ PV and $0.12 \mathrm{~nm}$ rms. These values are worse than those in section 4 due to both numerical error in the integration process and air current in the experimental atmosphere. Measurement reproducibility with rotation of the mirror is evaluated by rotating the test mirror about its optical axis. The results before and after a 90-degree 
rotation of the mirror exhibit a reproducibility of $2.0 \mathrm{~nm} \mathrm{PV}$ and $0.85 \mathrm{~nm} \mathrm{rms}$ in the two reconstructed datasets.

Trial measurements of several aspherical surfaces using our PS/PDI system are described and discussed. The surfaces studied were a spherical concave mirror having large asphericity, an off-axis parabolic surface, and a silicon wafer as a flat surface. The results increased the availability of our PS/PDI system for aspherical surfaces. The test concave mirror has a large deviation from an ideal sphere, and the focal point is not clear. Therefore, locations of the two fiber ends and CCD are determined by procedures described in sections 4 and 6, and the wavefront reconstruction process is performed using the location data. The mirror surface figure is calculated by averaging efficient data in 100 consecutive measurements. Measurement reproducibility evaluated from two data before and after a mirror rotation is $10 \mathrm{~nm} \mathrm{PV}$ and $1.4 \mathrm{~nm} \mathrm{rms}$. In the measurement of the parabolic mirror, the fiber end for the test wave is set at the focal point of the surface, and the reflected or collimated test wavefront interfered with the reference wave. The resultant wavefront has a reproducibility of $10 \mathrm{~nm}$ PV and $1.3 \mathrm{~nm}$ rms. Misalignment of the fiber end for the test wave is studied and appearance of low-frequency error is evaluated. In the measurement of the silicon wafer, the diffraction wavefront reflected by the wafer surface is measured using the PS/PDI because the wave includes information of the wafer surface figure. The wavefront deviation data from an ideal sphere is converted to the surface figure of the silicon wafer by the ray trace method. The resultant figure data agrees well with data from a white-light interferometer in the low-frequency components.

\section{Acknowledgment}

A part of this work was supported by a Grants-in-Aid for Scientific Research (KAKENHI) (22560109, 19360066, 16360067) from Japan Society for the Promotion of Science (JSPS). Authors express their sincere gratitude to Dr. Toshiaki Matsuura (Mitsutoyo Corporation) for his intense study in doctoral course. Finally authors are grateful to editors for giving them the chance to contribution to this book.

\section{References}

Ando, M. and 67 co-authors (2001), Stable Operation of a 300-m Laser Interferometer with Sufficient Sensitivity to Detect Gravitational-Wave Events within Our Galaxy, Phys. Rev. Lett. 86, pp. 3950-3954.

Born, M. and Wolf, M. (1959), Principles of Optics. Pergamon Press: London, New York, Paris, Los Angeles.

Chen, S., Li, S., Dai, Y., and Zheng, Z. (2006), Iterative algorithm for subaperture stitching test with spherical interferometers, J. Opt. Soc. Am. A 23, n. 5, pp. 1219-1226.

Creath, K. and Hariharan, P. (1994), Phase-shifting errors in interferometric tests with highnumerical-aperture reference surfaces, APPLIED OPTICS, Vol. 33, No. 1, pp. 2425.

de Groot, P. (1995), Derivation of algorithms for phase-shifting interferometry using the concept of a data-sampling window, Applied Optics Vol. 34, No. 22, pp. 47234730. 
de Groot, P. (1995), Phase-shift calibration errors in interferometers with spherical Fizeau cavities, Applied Optics Vol. 34, No. 22, pp. 2856-2863.

de Groot, P. J. (1995), Vibration in phase-shifting interferometry, J. Opt. Soc. Am. A, Vol. 12, No. 2, pp. 354-365.

Ferraro, P., Del Core, C., Miccio, L., Grilli, S., De Nicola, S., Finizio, A. and Coppola, G. (2007), Phase map retrieval in digital holography: avoiding the undersampling effect by a lateral shear approach, Optics Letters, Vol. 32, No. 15, pp. 22332235.

Freischlad, K. and Koliopoulos, C. L. (1990), Fourier description of digital phase-measuring interferometry, J. Opt. Soc. Am. A/Vol. 7, No. 4, pp. 542-551.

Goldberg, K. A., Naulleau, P. P., Denham, P. E., Rekawa, S. B., Jackson, K., Anderson, E. H., and Liddle, J. A. (2004), At-wavelength alignment and testing of the 0.3 NA MET optic, J. Vac. Sci. Technol. B 22, pp. 2956-2961.

Greivenkamp, J. E. (1987), Sub-Nyquist interferometry, Applied Optics, vol. 26, n. 24, pp. 5245-5258.

Handa, S., Mimura, H., Yumoto, H., Kimura, T., Matsuyama, S., Sano, Y. and Yamauchi, K. (2008), Highly accurate differential deposition for X-ray reflective optics. Surface and Interface Analysis, 40: pp. 1019-1022.

Harris, F. J. (1978), On the use of windows for harmonic analysis with the discrete Fourier transform, Proceedings of the IEEE, Volume: 66 Issue:1, pp. 51-83.

Higashi, Y., Endo, K., Kume, T., Enami, K., Uchikoshi, J., Ueno, K., Mori, Y. (2007),Surface gradient integrated profiler for $\mathrm{x}$-ray and EUV optics: self calibration method of measured position for an off-axis parabolic mirror $(\mathrm{f}=150 \mathrm{~mm})$ measurement, Proc. of SPIE vol. 6704, 67040D.

Iemmi, C., Moreno, A., and Campos, J. (2005), Digital holography with a point diffraction interferometer, OPTICS EXPRESS, Vol. 13, No. 6, pp. 1885-1891.

Kazuya Ota; Takahiro Yamamoto; Yusuke Fukuda; Katsura Otaki; Iwao Nishiyama; Shinji Okazaki (2001), Advanced point diffraction interferometer for EUV aspherical mirrors (Proceedings Paper), Proceedings of SPIE Vol. 4343, pp.543-550.

Larkin, K. G. and Oreb, B. F. (1992), Design and assessment of symmetrical phase-shifting algorithms, J. Opt. Soc. Am. A, Vol. 9, No. 10, pp. 1740-1748.

Matsuura, T., Okagaki, S., Nakamura, T., Oshikane, Y., Inoue, H., Nakano, M., and Kataoka (2007), T., Measurement Accuracy in Phase-Shifting Point Diffraction Interferometer with Two Optical Fibers, OPTICAL REVIEW Vol. 14, No. 6, pp. 401405.

Matsuura, T., Okagaki, S., Oshikane, Y., Inoue, H., Nakano, M. and Kataoka, T. (2008), Numerical reconstruction of wavefront in phase-shifting point diffraction interferometer by digital holography. Surface and Interface Analysis, 40: 10281032.

Matsuura, T., Udaka, K., Oshikane, Y., Inoue, H., Nakano, M., Yamauchi, K., Kataoka, T. (2010), Spherical concave mirror measurement by phase-shifting point diffraction interferometer with two optical fibers, Nuclear Instruments and Methods in Physics Research A 616, pp. 233-236. 
Matsuyama, S., Mimura, H., Katagishi, K., Yumoto, H., Handa, S., Fujii, M., Sano, Y., Shimura, M., Yabashi, M., Nishino, Y., Tamasaku, K., Ishikawa, T. and Yamauchi, K. (2008), Trace element mapping using a high-resolution scanning $X$-ray fluorescence microscope equipped with a Kirkpatrick-Baez mirror system. Surface and Interface Analysis, 40: pp. 1042-1045.

Medecki, H., Tenjnil, E., Goldberg, K. A., \& Bokor, J. (1996), Phase-shifting point diffraction interferometer, Optics Letters, Vol. 21, Iss. 19, pp. 1526-1528.

Millerd, J. E., Brock, N. J., Hayes, J. B., Wyant, J. C. (2004), Instantaneous phase-shift pointdiffraction interferometer, Proceedings of SPIE, Vol. 5531, pp. 264-272.

Nomura, T., Murata, S., Nitanai, E., and Numata, T. (2006), Phase-shifting digital holography with a phase difference between orthogonal polarizations, Applied Optics Vol. 45, No. 20, pp. 4873-4877.

Oshikane, Y., Kataoka, T., Endo, K., Noguchi, A., Inoue, H. and Nakano, M. (2003), Development of Phase-Shifting Point Diffraction Interferometry with Fiber Point Sources, J. of the Japan Society of Precision Engineering 69, pp. 678-682.

Otaki, K., Ota, K., Nishiayama, I., Yamamoto, T., Fukuda, \& Y., Okazaki, S. (2002). Development of the point diffraction interferometer for extreme ultraviolet lithography: Design, fabrication, and evaluation, J. Vac. Sci. Technol. B 20, pp. 24492458 (2002).

Otaki, K., Yamamoto, T., Fukuda, T., Ota, K., Nishiyama, I., and Okazaki, S. (2002), Accuracy evaluation of the point diffraction interferometer for extreme ultraviolet lithography aspheric mirror, J. Vac. Sci. Technol. B 20, pp. 295-300.

Sato, S., Miyoki, S., Ohashi, M., Fujimoto, M., Yamazaki, T., Fukushima, M., Ueda, A., Ueda, K., Watanabe, K., Nakamura, K., Etoh, K., Kitajima, N., Ito, K., and Kataoka, I. (1999), Loss factors of mirrors for a gravitational wave antenna, APPLIED OPTICS, Vol. 38, No. 13, pp. 2880-2885.

Schulz, G. and Schwider, J. (1967), Precise Measurement of Planeness, Applied Optics vol. 6, n. 6, pp. 1077-1084.

Smartt, R. N. and Strong, J. (1972), Point-Diffraction Interferometer, Journal of the Optical Society of America, 62, p. 737.

Smartt, R. N., and Steel, W. H. (1975), Theory and Application of Point-Diffraction Interferometers, Jpn. J. Appl. Phys. 14 Supplement 14-1 pp. 351-356.

Sommargren, G. E. (1996), Phase Shifting Diffraction Interferometry for Measuring Extreme Ultraviolet Optics, OSA TOPS on Extreme Ultraviolet Lithography, Vol. 4,

Stulen, R.H. \& Sweeney, D.W. (1999). Extreme ultraviolet lithography, IEEE J. of Quantum Electronics, vol. 35, pp. 694-699 (1999).

van Wingerden, J., Frankena, H. J., and Smorenburg, C. (1991), Linear approximation for measurement errors in phase shifting interferometry, APPLIED OPTICS, Vol. 30, No. 19, pp. 2718-2729.

Yamaguchi, I., Ida, T., Yokota, M., and Yamashita, K. (2006), Surface shape measurement by phase-shifting digital holography with a wavelength shift, Applied Optics Vol. 45, No. 29, pp. 7610-7616. 
Yamamura, K. and Takai, H. (2008), Figuring of elliptical hard X-ray focusing mirror using 1-dimensional numerically controlled local wet etching. Surface and Interface Analysis, 40: pp. 1014-1018.

Yumoto, H., Mimura, H., Kimura, T., Handa, S., Matsuyama, S., Sano, Y. and Yamauchi, K. (2008), Stitching interferometric metrology for steeply curved x-ray mirrors. Surface and Interface Analysis, 40: pp. 1023-1027. 




\author{
Selected Topics on Optical Fiber Technology \\ Edited by Dr Moh. Yasin
}

ISBN 978-953-51-0091-1

Hard cover, 668 pages

Publisher InTech

Published online 22, February, 2012

Published in print edition February, 2012

This book presents a comprehensive account of the recent advances and research in optical fiber technology. It covers a broad spectrum of topics in special areas of optical fiber technology. The book highlights the development of fiber lasers, optical fiber applications in medical, imaging, spectroscopy and measurement, new optical fibers and sensors. This is an essential reference for researchers working in optical fiber researches and for industrial users who need to be aware of current developments in fiber lasers, sensors and other optical fiber applications.

\title{
How to reference
}

In order to correctly reference this scholarly work, feel free to copy and paste the following:

Oshikane Yasushi, Nakano Motohiro and Inoue Haruyuki (2012). Phase-Shifting Point Diffraction Interferometer Having Two Point Light Sources of Single-Mode Optical Fibers, Selected Topics on Optical Fiber Technology, Dr Moh. Yasin (Ed.), ISBN: 978-953-51-0091-1, InTech, Available from: http://www.intechopen.com/books/selected-topics-on-optical-fiber-technology/phase-shifting-point-diffractioninterferometer-having-two-point-light-sources-of-single-mode-optica

\section{INTECH}

open science | open minds

\section{InTech Europe}

University Campus STeP Ri

Slavka Krautzeka 83/A

51000 Rijeka, Croatia

Phone: +385 (51) 770447

Fax: +385 (51) 686166

www.intechopen.com

\section{InTech China}

Unit 405, Office Block, Hotel Equatorial Shanghai

No.65, Yan An Road (West), Shanghai, 200040, China

中国上海市延安西路65号上海国际贵都大饭店办公楼405单元

Phone: +86-21-62489820

Fax: +86-21-62489821 
(C) 2012 The Author(s). Licensee IntechOpen. This is an open access article distributed under the terms of the Creative Commons Attribution 3.0 License, which permits unrestricted use, distribution, and reproduction in any medium, provided the original work is properly cited. 